University of Tennessee Health Science Center UTHSC Digital Commons

\title{
$5-2019$
}

\section{Study of Natural Killer Cells and Their Therapeutic Role in Pediatric Cancer}

\author{
Hong Ha Rosa Nguyen \\ University of Tennessee Health Science Center
}

Follow this and additional works at: https://dc.uthsc.edu/dissertations

Part of the Oncology Commons

\section{Recommended Citation}

Nguyen, Hong Ha Rosa (0000-0001-5143-2055), "Study of Natural Killer Cells and Their Therapeutic Role in Pediatric Cancer" (2019). Theses and Dissertations (ETD). Paper 488. http://dx.doi.org/10.21007/ etd.cghs.2019.0480.

This Dissertation is brought to you for free and open access by the College of Graduate Health Sciences at UTHSC Digital Commons. It has been accepted for inclusion in Theses and Dissertations (ETD) by an authorized administrator of UTHSC Digital Commons. For more information, please contact jwelch30@uthsc.edu. 


\title{
Study of Natural Killer Cells and Their Therapeutic Role in Pediatric Cancer
}

\begin{abstract}
NK cells are known to play an important role in the natural defense against viral infections and tumor immune surveillance. Through complex interactions between NK cell receptors and target cell ligands, transformed or unhealthy cells are identified and rapidly eliminated. NK cells have been used for therapeutic purposes in pediatric oncology, for example by harnessing the mechanisms of NK cell surveillance in acute myeloid leukemia (AML) or intervention-augmented antibody-dependent cellmediated cytotoxicity (ADCC) in neuroblastoma. However, the underlying cellular mechanisms in these processes are not fully understood. In my thesis research, I sought to gain a deeper understanding of the molecular and cellular changes within NK cells that occur in the tumor microenvironment and with immunotherapy. I discovered that NK cells have attenuated natural cytotoxicity in children with neuroblastoma that correlates with clinical tumor response to chemoimmunotherapy. Compared to agematched reference data in healthy children, the studied patients had higher proportions of CD56bright NK cells, suggestive of immaturity of the NK cell compartment. Although preactivation with cytokines did not entirely overcome the hyporeactivity in patient NK cells, the therapeutic use of interleukin (IL)-2 or -15 significantly enhanced the natural cytotoxicity and ADCC of tested NK cells against neuroblastoma in vitro and in vivo and therefore warrants further investigation. To provide a suitable animal model for future studies of NK cell dysfunction and immunotherapy, I developed a humanize MISTRG neuroblastoma model. Human NK cells that arise in this model after hematopoietic progenitor cell transplantation were functionally intact and capable of suppressing neuroblastoma growth with chemoimmunotherapy in vivo. Despite the discovered molecular differences of NK cell subsets in these mice compared to patient NK cells, the humanized MISTRG neuroblastoma model is a valuable tool to study NK cell biology in neuroblastoma and test whether therapeutic interventions, such as cytokine supplementation, can overcome impaired NK cytotoxicity. Collectively, results from this work are important because they link the cellular capacity of a key effector cell involved in ADCC to antibodymediated tumor shrinkage in the clinic, revealing potential mechanisms for therapy failure in patients who receive chemoimmunotherapy for neuroblastoma.
\end{abstract}

\section{Document Type}

Dissertation

Degree Name

Doctor of Philosophy (PhD)

Program

Biomedical Sciences

Research Advisor

Michael A. Dyer, Ph.D.

Keywords

Humanized mice, Neuroblastoma, NK cells, Pediatric Oncology

Subject Categories

Medicine and Health Sciences | Oncology 


\title{
Study of Natural Killer Cells and Their Therapeutic Role in Pediatric Cancer
}

\author{
A Dissertation \\ Presented for \\ The Graduate Studies Council \\ The University of Tennessee \\ Health Science Center \\ In Partial Fulfillment \\ Of the Requirements for the Degree \\ Doctor of Philosophy \\ From The University of Tennessee
}

\author{
By \\ Hong Ha Rosa Nguyen \\ May 2019
}


Portions of Chapter 2, 3, and 4 (C) 2018 by Springer Nature. All other material (C) 2019 by Hong Ha Rosa Nguyen.

All rights reserved. 


\section{DEDICATION}

To my patients who have stood up to cancer with resilience, strength, and bravery. 


\section{ACKNOWLEDGEMENTS}

I would like to express my gratitude to my mentor, Dr. Michael Dyer, for

providing me the opportunity and support to pursue my $\mathrm{PhD}$ in his laboratory and guiding me through this experience.

I would also like to thank my dissertation committee members, Drs. Terrence Geiger, Peter Murray, Marko Radic, Jeffrey Rubnitz, and Paul Thomas for their invaluable scientific advice and direction.

I am grateful for my lab members who have been very supportive of my research endeavors. In addition, I would like to thank Jim Houston who shared his expertise in flow cytometry with me; Dr. Wayne Furman for allowing me to conduct the correlative biology studies on his clinical trial; Dr. Mitchell Weiss for kindly providing hematopoietic stem and progenitor cells for my experiments; Dr. Ardiana Moustaki for scientific discussions; and my external collaborators and supporters, Drs. Michael Caligiuri and Wing Chan, Drs. Paul Sondel and Jackie Hank, and Dr. Dan Kaufman.

I would like to thank the Conquer Cancer ASCO Foundation for the Young Investigator Award that provided financial funds for part of my dissertation research.

I extend my deepest gratitude to my parents, Kim Hoa and Thanh Nguyen, and my brother, Charles Nguyen, for the sacrifices they made that allowed me to have access to unparalleled educational opportunities. Lastly, my achievements would not have been possible without the unconditional support from my husband, Christian Okoye. 


\begin{abstract}
NK cells are known to play an important role in the natural defense against viral infections and tumor immune surveillance. Through complex interactions between NK cell receptors and target cell ligands, transformed or unhealthy cells are identified and rapidly eliminated. NK cells have been used for therapeutic purposes in pediatric oncology, for example by harnessing the mechanisms of NK cell surveillance in acute myeloid leukemia (AML) or intervention-augmented antibody-dependent cell-mediated cytotoxicity (ADCC) in neuroblastoma. However, the underlying cellular mechanisms in these processes are not fully understood. In my dissertation research, I sought to gain a deeper understanding of the molecular and cellular changes within NK cells that occur in the tumor microenvironment and with immunotherapy. I discovered that NK cells have attenuated natural cytotoxicity in children with neuroblastoma that correlates with clinical tumor response to chemoimmunotherapy. Compared to age-matched reference data in healthy children, the studied patients had higher proportions of CD56 $6^{\text {bright }} \mathrm{NK}$ cells, suggestive of immaturity of the NK cell compartment. Although preactivation with cytokines did not entirely overcome the hyporeactivity in patient NK cells, the therapeutic use of interleukin (IL)-2 or -15 significantly enhanced the natural cytotoxicity and ADCC of tested NK cells against neuroblastoma in vitro and in vivo and therefore warrants further investigation. To provide a suitable animal model for future studies of NK cell dysfunction and immunotherapy, I developed a humanize MISTRG neuroblastoma model. Human NK cells that arise in this model after hematopoietic progenitor cell transplantation were functionally intact and capable of suppressing neuroblastoma growth with chemoimmunotherapy in vivo. Despite the discovered molecular differences of NK cell subsets in these mice compared to patient NK cells, the humanized MISTRG neuroblastoma model is a valuable tool to study NK cell biology in neuroblastoma and test whether therapeutic interventions, such as cytokine supplementation, can overcome impaired NK cytotoxicity. Collectively, results from this work are important because they link the cellular capacity of a key effector cell involved in ADCC to antibody-mediated tumor shrinkage in the clinic, revealing potential mechanisms for therapy failure in patients who receive chemoimmunotherapy for neuroblastoma.
\end{abstract}




\section{TABLE OF CONTENTS}

CHAPTER 1. INTRODUCTION .....................................................................................1

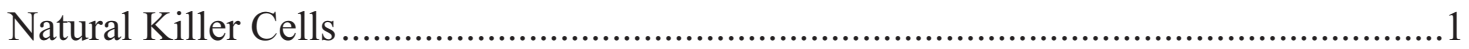

Physiologic role of natural killer cells in innate immunity ......................................

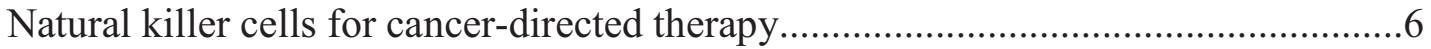

Preclinical Animal Models for Immunotherapy Testing ....................................11

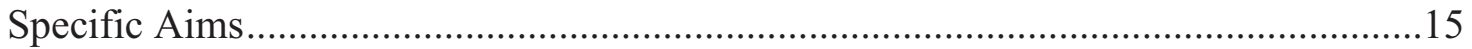

Objective 1: To elucidate the cellular mechanism underlying ADCC in

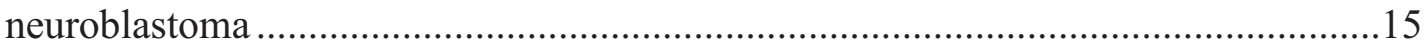

Objective 2: To develop a humanized mouse model and validate its use for

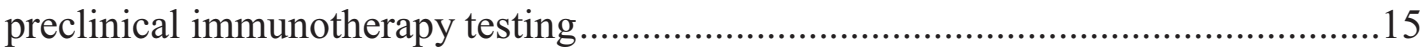

Objective 3: To relate preclinical findings to correlative biology studies in children with AML and neuroblastoma ........................................................... 16

CHAPTER 2. METHODOLOGY ...........................................................................17

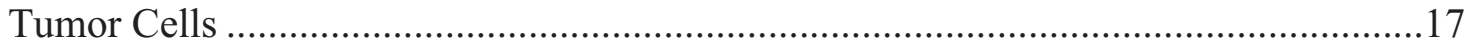

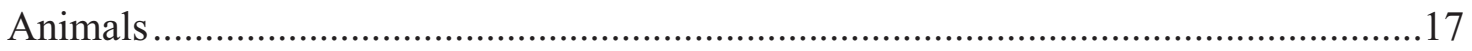

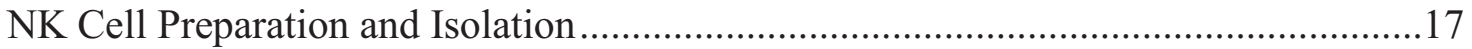

Hematopoietic Stem and Progenitor Cells .......................................................... 18

Transplantation Procedures.................................................................................. 18

Monoclonal Therapeutic Antibodies .....................................................................18

ADCC and NK Cytotoxicity Assays and Confluence Analysis .................................19

CD107a Degranulation Assay .................................................................................19

Sphere Formation and Infiltration Assay ............................................................... 19

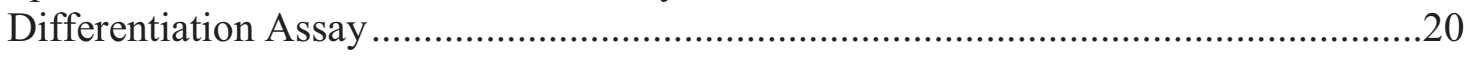

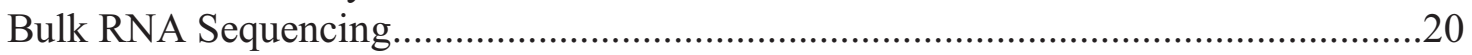

Single-Cell RNA Sequencing Data Processing and Analysis ..................................20

HLA Genotyping and Definition of KIR-HLA Mismatch......................................21

Flow Cytometry and Fluorescence-Activated Cell Sorting (FACS) ............................21

Preclinical Immunotherapy Testing In Vivo ............................................................22

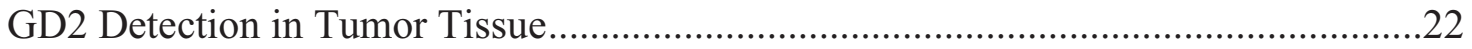

Patient Enrollment Criteria for NB2012 Study......................................................22

NK Cell Collection and Treatment Regimen of the NB2012 Study..........................23

Correlative Biology Studies on the NB2012 Study ..................................................23

Patient and Donor Enrollment Criteria for AML-NK Trial.........................................23

NK Cell Collection and Treatment Regimen of the AML-NK Trial...........................24

Correlative Biology Studies on the AML-NK Trial .................................................24

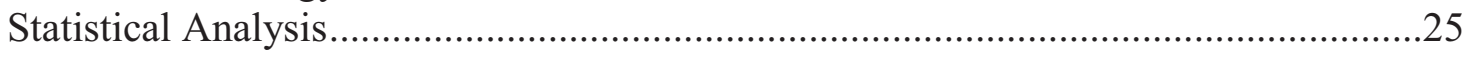

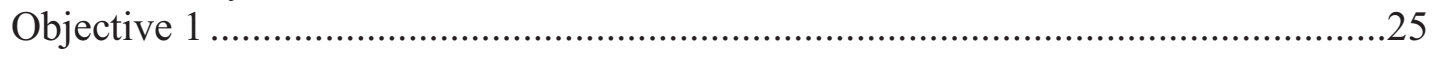

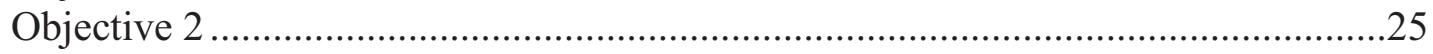

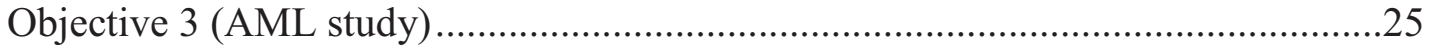

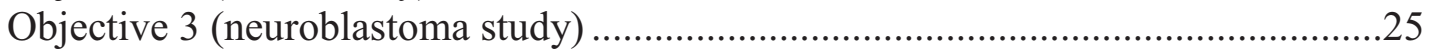


Objective 1

Development and validation of an assay for NK cell-mediated cytotoxicity

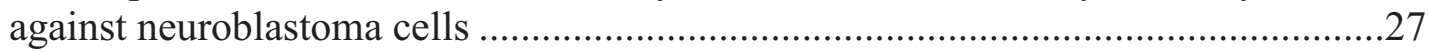

IL-2 improves ADCC and NK cell-mediated cytotoxicity .................................29

ADCC is more effective than "Missing-self" for NK cell degranulation ..................29

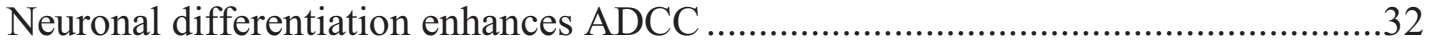

Individualized testing of ADCC is possible with PDXs and matched NK cells

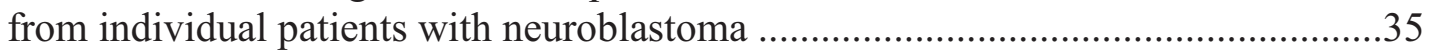

IL-15 enhances natural cytotoxicity and ADCC in neuroblastoma ..........................35

IL-15 increases the number of tumor-infiltrating NK cells in neuroblastoma

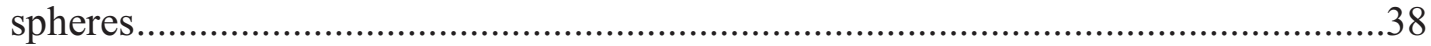

The IL-15/IL-15R $\alpha$-complex suppresses growth of neuroblastoma PDX in vivo.....41

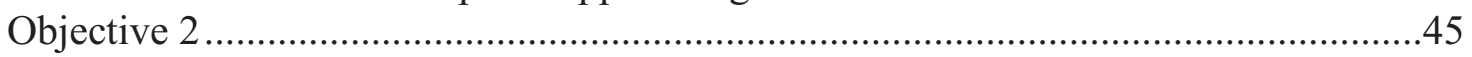

Engraftment and function of MISTRG-derived NK cells...................................45

Double-transplantation protocol in adult MISTRG mice .....................................45

Molecular and functional analyses of NK cells derived from MISTRG mice with

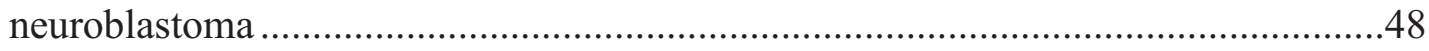

Comparison of adoptive NK cells with endogenously produced human NK cells

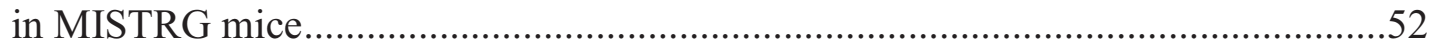

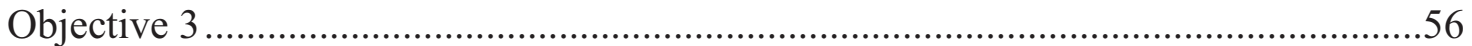

A phase II clinical trial of adoptive transfer of haploidentical natural killer cells for consolidation therapy of pediatric AML ........................................................56

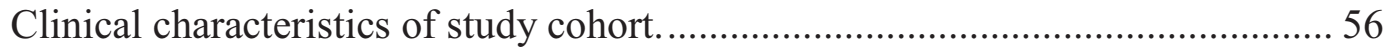

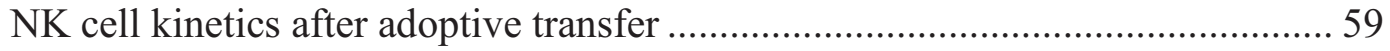

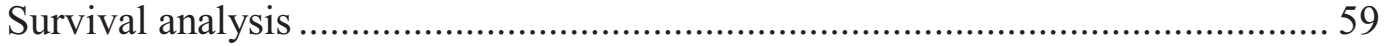

Analysis of NK cell phenotype and reconstitution in children treated with

combined chemoimmunotherapy for neuroblastoma............................................62

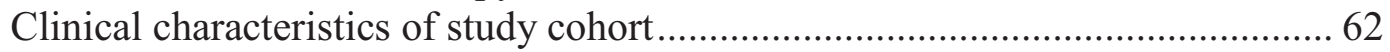

NK cell kinetics during chemoimmunotherapy ...............................................6 62

NK cell reconstitution during consolidation with haploidentical NK cell infusion

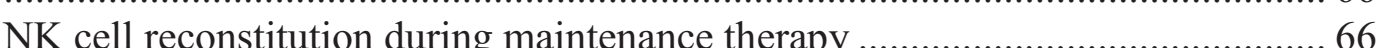

NK cells from patients with neuroblastoma are dysfunctional ...............................69

Molecular analyses of NK cells derived from patients with neuroblastoma ............69

CHAPTER 4. DISCUSSION ............................................................................................75

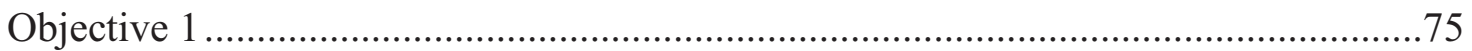

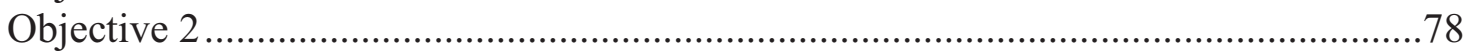

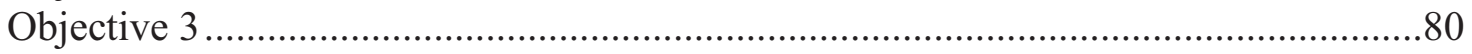

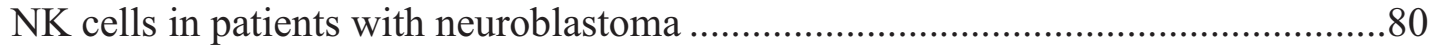

Adoptive NK cell transfer for AML therapy ...................................................... 82

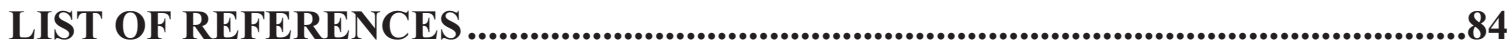

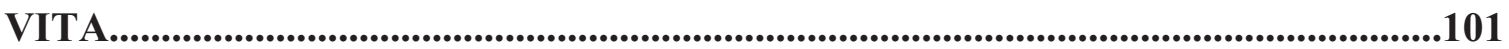




\section{LIST OF TABLES}

Table 1-1. Inhibitory and activating NK cell receptors and cognate ligands .................5

Table 3-1. Clinical characteristics of patients who received NK cell therapy ...............58

Table 3-2. Clinical characteristics of studied patient cohort ...................................64 


\section{LIST OF FIGURES}

Figure 1-1. Mechanisms of target cell recognition and elimination by NK cells.............4

Figure 1-2. Mechanisms of tumor cell detection and evasion to escape NK cell recognition.

Figure 1-3. Common variables of mouse models of neuroblastoma for preclinical immunotherapy testing

Figure 3-1. Development and validation of an assay to quantify NK cell--mediated cytotoxicity and ADCC in neuroblastoma cells. .28

Figure 3-2. Development of an assay to quantify NK cell-mediated cytotoxicity and ADCC in PDXs . 30

Figure 3-3. IL-2 enhances NK cell-mediated ADCC in neuroblastoma cells

Figure 3-4. Comparison of ADCC and missing-self recognition for neuroblastoma cell ablation

Figure 3-5. Effect of neuroblastoma differentiation on NK cell-mediated ADCC .........36

Figure 3-6. ADCC assays with matched O-PDXs and patient NK cells .....

Figure 3-7. Effects of IL-2 and IL-15 on natural cytotoxicity against neuroblastoma and tumor-NK cell contact

Figure 3-8. Phenotype and function of tumor-sphere-infiltrating NK cells (SPHINKs)

Figure 3-9. Comparison of IL-15/IL-15Ra-complex with IL-2 as immunoadjuvant agent in immunotherapy for neuroblastoma....

Figure 3-10. NK cell engraftment and function in MISTRG mice after perinatal transplantation

Figure 3-11. Modified transplantation protocol to induce double-engraftment with human hematopoiesis and tumor in adult MISTRG mice

Figure 3-12. Work flow of single-cell RNA sequencing......

Figure 3-13. Single-cell transcriptome analysis of NK cells from humanized MISTRG mice with neuroblastoma .50

Figure 3-14. Comparison of adoptive NK cells with endogenously produced human NK cells in MISTRG mice. 
Figure 3-15. Therapy regimen of patients with AML

Figure 3-16. NK cell kinetics in patients after adoptive transfer of haploidentical KIR-HLA-mismatched NK cells ....

Figure 3-17. Survival curves and NK cell dose distribution

Figure 3-18. Therapy regimen of patients with high-risk neuroblastoma and time points of phenotype testing

Figure 3-19. Box plot of total and $\mathrm{CD} 16^{+} \mathrm{NK}$ cells (logarithmic scale) by time point.....67

Figure 3-20. Individual alloreactive NK cell counts on days 7 and 21 after adoptive transfer of haploidentical and KIR-HLA-mismatched NK cells .68

Figure 3-21. Ex vivo cytotoxic activity of NK cells from patients with neuroblastoma and healthy controls.

Figure 3-22. Single-cell transcriptome analysis of NK cells from patients with neuroblastoma

Figure 3-23. Single-cell transcriptome analysis of NK cells from patients with neuroblastoma after therapy..... 


\section{LIST OF ABBREVIATIONS}

\begin{tabular}{|c|c|}
\hline ADCC & Antibody-dependent cell-mediated cytotoxicity \\
\hline $\mathrm{AE}$ & Adverse events \\
\hline AML & Acute myeloid leukemia \\
\hline ATRA & all-trans retinoic acid \\
\hline BLT & Bone marrow-liver-thymus \\
\hline $\mathrm{BMT}$ & Bone marrow transplantation \\
\hline CAR & Chimeric antigen receptor \\
\hline CNS & Central nervous system \\
\hline CPM & Cyclophosphamide \\
\hline DAPI & 4',6-diamidino-2-phenylindole \\
\hline DIC & Differential interference contrast \\
\hline FACS & Fluorescence-activated cell sorting \\
\hline $\mathrm{F}_{\mathrm{c}}$ & Fragment, crystallizable \\
\hline $\mathrm{F}_{\mathrm{c}} \mathrm{Rn}$ & Neonatal $F_{c}$ receptor \\
\hline FLU & Fludarabine \\
\hline GD2 & Disialogangloside \\
\hline GM-CSF & Granulocyte-macrophage colony-stimulating factor \\
\hline GVHD & Graft-versus-host disease \\
\hline HLA & Human leukocyte antigen \\
\hline IFN- $\gamma$ & Interferon gamma \\
\hline $\operatorname{IgG}$ & Immunoglobulin $\mathrm{G}$ \\
\hline IL & Interleukin \\
\hline IL- $15 \mathrm{R} \alpha$ & IL-15 receptor $\alpha$-subunit \\
\hline INSS & International Neuroblastoma Staging System \\
\hline KIRs & Killer-cell immunoglobulin-like receptors \\
\hline KLRB1 & Killer cell lectin-like receptor subfamily B, member 1 \\
\hline LOA & List of Abbreviations (sample $2^{\text {nd }}$ entry) \\
\hline MAGE-A3 & Melanoma-associated antigen 3 \\
\hline $\mathrm{MHC}$ & Major histocompatibility complex \\
\hline MIC & HLA class I chain-related \\
\hline MISTRG & $M-C S F^{\mathrm{h} / \mathrm{h}} I L-3 / G M-C S F^{\mathrm{h} / \mathrm{h}} S I R P A^{\mathrm{h} / \mathrm{m}} T P O^{\mathrm{h} / \mathrm{h}} \operatorname{Rag} 2^{\text {null }} / I l 2 R \gamma_{c}{ }^{\text {null }}$ \\
\hline MRD & Minimal residual disease therapy phase. \\
\hline $\mathrm{N} / \mathrm{A}$ & Not available \\
\hline NCR & Natural cytotoxicity receptors \\
\hline NK & Natural killer \\
\hline NSG & $N O D /$ scid $/ I l 2 R \gamma_{c}^{\text {null }}$ \\
\hline PBMC & Peripheral blood mononuclear cells \\
\hline PBS & Phosphate-buffered saline \\
\hline PDX & Patient-derived xenograft \\
\hline RG & $\operatorname{Rag} 2^{\text {null }} / I l 2 R \gamma_{c}{ }^{\text {null }}$ \\
\hline RLU & Relative light units \\
\hline SGM-3 & CMV-IL3,CSF2,KITLG \\
\hline SIRPA & Signal regulatory protein alpha \\
\hline
\end{tabular}


SPHINKs

TCR

TGF- $\beta 1$

TIGIT

TILs

TNF- $\alpha$

$\mathrm{t}$-SNE

UPN
Sphere-infiltrating NK cells

T cell receptor

Transforming growth factor beta 1

$\mathrm{T}$ cell immunoglobulin and immunoreceptor tyrosine

Tumor-infiltrating $\mathrm{T}$ lymphocytes

Tumor necrosis factor alpha

t-distributed Stochastic Neighbor Embedding

Unique patient number. 


\section{CHAPTER 1. INTRODUCTION}

\section{Natural Killer Cells}

\section{Physiologic role of natural killer cells in innate immunity}

Natural killer (NK) cells are large granular lymphocytes of the innate immune system and broadly divided into two main phenotypic subgroups based on the cell surface expression of CD56 [1]. CD56 ${ }^{\mathrm{dim}} \mathrm{NK}$ cells predominate in the peripheral blood, spleen, and bone marrow and co-express $\mathrm{CD} 16$, the receptor that recognizes the $\mathrm{F}_{\mathrm{c}}$ (fragment, crystallizable) region of immunoglobulin $\mathrm{G}(\mathrm{IgG})$ antibodies $[2,3]$. They are putatively regarded as the main effector cells in natural and antibody-dependent cell-mediated cytotoxicity (ADCC), whereas CD56 ${ }^{\text {bright }}$ CD16- NK cells, enriched in secondary lymphoid tissues, produce and secrete cytokines and chemokines to orchestrate an inflammatory response $[2,4,5]$.

NK cells account for about 5-15\% of the peripheral blood lymphocytes and have an average life span of approximately two weeks [6]. Compared to cells with adaptive immunity that can clonally expand and retract, mature NK cells have a very limited ability to proliferate. Even during infection, such as acute mononucleosis, when overall NK cell numbers are significantly elevated, NK cell kinetics remain largely unchanged $[6,7]$. The primary site of human NK-cell production is in the bone marrow, while NK cell maturation occurs in secondary lymphoid tissues, such a lymph nodes and tonsils [2]. It has been previously hypothesized that $\mathrm{CD}_{5} 6^{\text {bright }} \mathrm{CD}^{-} 6^{-} \mathrm{NK}$ cells are developmental precursors to the terminally differentiated CD56 ${ }^{\mathrm{dim}} \mathrm{CD} 16^{+}$sub-group [8]. This is because lymph nodes and tonsils are enriched for $\mathrm{CD} 56^{\text {bright }} \mathrm{CD} 16^{-} \mathrm{NK}$ cells, and because these cells up-regulate the expression of natural cytotoxicity receptors (NCRs), CD16, and perforin following co-culture with interleukin (IL)-2, thereby attaining a CD56 ${ }^{\text {dim-like }}$ phenotype $[9,10]$. However, a more recent study has challenged this assumption using an autologous bone marrow transplantation model in the rhesus macaque with quantitative genetic barcoding to track the clonal evolution of hematopoiesis including NK cell development over time [11]. At 3 and 6.5 months following transplantation, the clonal composition of peripheral blood CD56- $\mathrm{CD}^{-} 6^{+} \mathrm{NK}$ cells was distinct from other blood lineages, whereas blood and lymph node-derived $\mathrm{CD}^{+} 6^{+} \mathrm{CD} 16^{-} \mathrm{NK}$ cells accounted for clones that converged towards T/B cells and granulocytes, suggesting both populations to be ontologically distinct.

Despite the wealth of studies that have phenotypically and molecularly characterized CD56 $6^{\text {bright }}$ and CD56 $6^{\text {dim }}$ NK cells [12], advances in understanding their physiologic role in health and disease have been limited by the differences of NK cell markers in mice compared to humans and the diversity of NK cell receptors in the population, impeding the development of preclinical animal models [13]. In this regard, fundamental insight was gained through patients with rare NK cell immunodeficiency syndromes characterized by quantitative (classical) or qualitative NK cell defects 
(functional) [14]. In classical NK cell deficiency that is caused by mutations in GATA2, IRF8, MCM4, or RTEL1, peripheral blood NK cells account for $<1 \%$ of lymphocytes and the proportion of NK cell subsets is skewed [15-18]. As an example, in patients with GATA2 deficiency, the CD56 $6^{\text {bright }}$ sub-population is virtually absent. But apart from NK cytopenia, CD56 ${ }^{\mathrm{dim}} \mathrm{NK}$ cells are also functionally impaired in these individuals. This could be due to a dual functional role of GATA2 in both subpopulations or interdependence of both subsets upon each other for intact cytotoxicity [19]. Functional NK cell deficiency is caused by biallelic mutations in FCGR3A that encodes CD16 [20, 21]. Patients with the pathognomonic L66H mutation have adequate development and distribution of NK cell subsets and normal ADCC, yet profoundly impaired natural cytotoxicity [22]. Because this mutation localizes to the distal Ig-like domain, there is no interference with binding to the $F_{c}$ portion of $\operatorname{IgG}$ antibodies that usually occurs through the proximal domain. However, the distal Ig-like domain stabilizes CD2 which is an important co-stimulatory signal for natural cytotoxicity in NK cells. Therefore, patients with functional NK cell deficiency have intact ADCC, yet impaired natural cytotoxicity [22]. Despite the molecular differences between the NK cell deficiency syndromes, affected individuals share an increased susceptibility to life-threatening infections with cytomegalovirus or herpes viruses that also pose an increased risk for virus-related malignancies [23].

To date, the largest population-based study that assessed the association of innate immunity with risk for cancer was conducted in Japan [24]. In a total of 3625 selfselected individuals who completed a detailed lifestyle questionnaire, cytotoxic activity of peripheral blood lymphocytes against major histocompatibility complex (MHC) Ideficient K562, an erythroleukemia cell line, was measured ex vivo, and participants were followed for 11 years with respect to the occurrence of cancer. Categorized by tertials, individuals with medium and high cytotoxic activity had a 0.6 -fold lower risk for the development of primary cancers than did those with low cytotoxicity when adjusted for age and cancer-promoting lifestyle behaviors. Because the cytotoxic activity was determined in peripheral blood lymphocytes, the investigators conducted a sub-group analysis with 52 participants to delineate the relative contribution of NK cells in this process. They demonstrated that low cytotoxic activity of lymphocytes was associated with decreased numbers of NK cells in the peripheral blood, decreased expression of the NK cell-specific activation receptor, killer cell lectin-like receptor subfamily B, member 1 (KLRB1), and increased levels of tumor necrosis factor alpha (TNF- $\alpha$ ) and interferon gamma (IFN- $\gamma$ ), concluding that NK cells are mainly responsible for ex vivo cytolysis of K562 in their experiments. Collectively, the clinical hallmarks of NK cell deficiency syndromes and the findings of this study infer that NK cells are indispensable for the containment of viral infections and immune surveillance of cancer.

Unlike T cells that gain receptor diversity and specificity through somatic VDJ rearrangements [25], NK cell diversity is determined by germline-encoded inhibitory and activating receptors that are expressed in various combinations and in multiple overlapping NK cell sub-populations [13]. These receptors are highly polymorphic and engage with cognate ligands in the body and allow the NK cell to select its appropriate target [26, 27]. Among the NK cell receptor family, CD94-NKG2 receptors and the 
killer-cell immunoglobulin-like receptors (KIRs) can recognize "self" by binding to human leukocyte antigen (HLA) class I molecules with differing affinities [28-30]. Because KIRs and HLAs map to different chromosomes and therefore are inherited separately, individuals may lack the HLA class I molecule corresponding to a KIR that is expressed on their own NK cell (autologous KIR-HLA-mismatch) [31]. Nevertheless, these individuals do not experience autoimmunity due to a complex "licensing" process that promotes the development of functional and self-tolerating NK cells [32-34]. In this process, inhibitory KIRs must engage HLA for the licensing of NK cells to occur. Conversely, NK cells whose KIRs never interacted with HLA during development remain in a quiescent unlicensed state, unless this hyporeactivity is later overcome through the engagement of activating receptors in the periphery. The KIR-HLAinteraction is regarded as the primary mechanism responsible for NK cell tolerance to self.

NK cell-mediated cytotoxicity is a tightly regulated process that enables licensed NK cells to identify and eliminate infected or transformed cells and spare normal tissue, thereby avoiding the devastating consequences of autoimmunity. Early experiments in murine models of bone marrow rejection demonstrated that NK cells do not require prior sensitization but mediate cytotoxicity against target cells that lack MHC class I expression ("missing self") as shown in Figure 1-1 [35, 36]. However, further investigations have also revealed that the mere absence of MHC class I molecules is necessary but not sufficient for cytolysis [37]. As such, in cases of $\beta 2$ microglobulindeficiency in mice or TAP-deficiency in humans and mice, NK cells are self-tolerant and can attenuate their cytotoxic activity [38-41]. Rather, it is understood now that NK cells induce target cell death based on the balance of inhibitory and activating signals from the multiple NK cell surface receptors after binding to their ligands [26]. Classic examples of inhibitory ligands and receptors are listed in Table 1-1 and include HLA class I molecules Group 1 and Group 2, HLA-Bw4, and the ubiquitously expressed HLA-E for inhibitory ligands. Activating ligands are HLA class I chain-related (MIC) molecules, MICA and MICB, and UL16-binding proteins [42-44]. The corresponding NK cell surface receptors for the above mentioned ligands are KIRs and receptors of the CD94/NKG2 family and KIRs with intracytoplasmic tails that confer NK cell activation rather than inhibition [26]. Normal healthy cells have a balance of inhibitory and activating ligands. As such, NK cell-mediated killing occurs when the activating ligand is up-regulated ("induced self") or the inhibitory ligand down-regulated (missing self) $[28,44,44]$. Both mechanisms can be found in cancer. For example, missing self is thought to occur in a variety of malignancies including neuroblastoma by downregulation of HLA class I molecules by which the tumor cell escapes T cell recognition but may be more susceptible to NK cell-mediated cytotoxicity [45]. In contrast, induced self is increased after cell damage or stress following chemotherapy or radiation therapy [46]. Once the NK cell has determined its target, it kills by releasing cytotoxic granules that contain granzymes and perforin, activating the death-receptor pathways on the target cell (e.g. TRAIL, Fas), or secreting IFN- $\gamma$ to upregulate MHC class I by activating JAK/STAT1 signaling as shown in Figure 1-1 [47]. In the clinic, oncologists have tried to harness the regulatory mechanisms of NK cells for cancer-directed immunotherapy. 

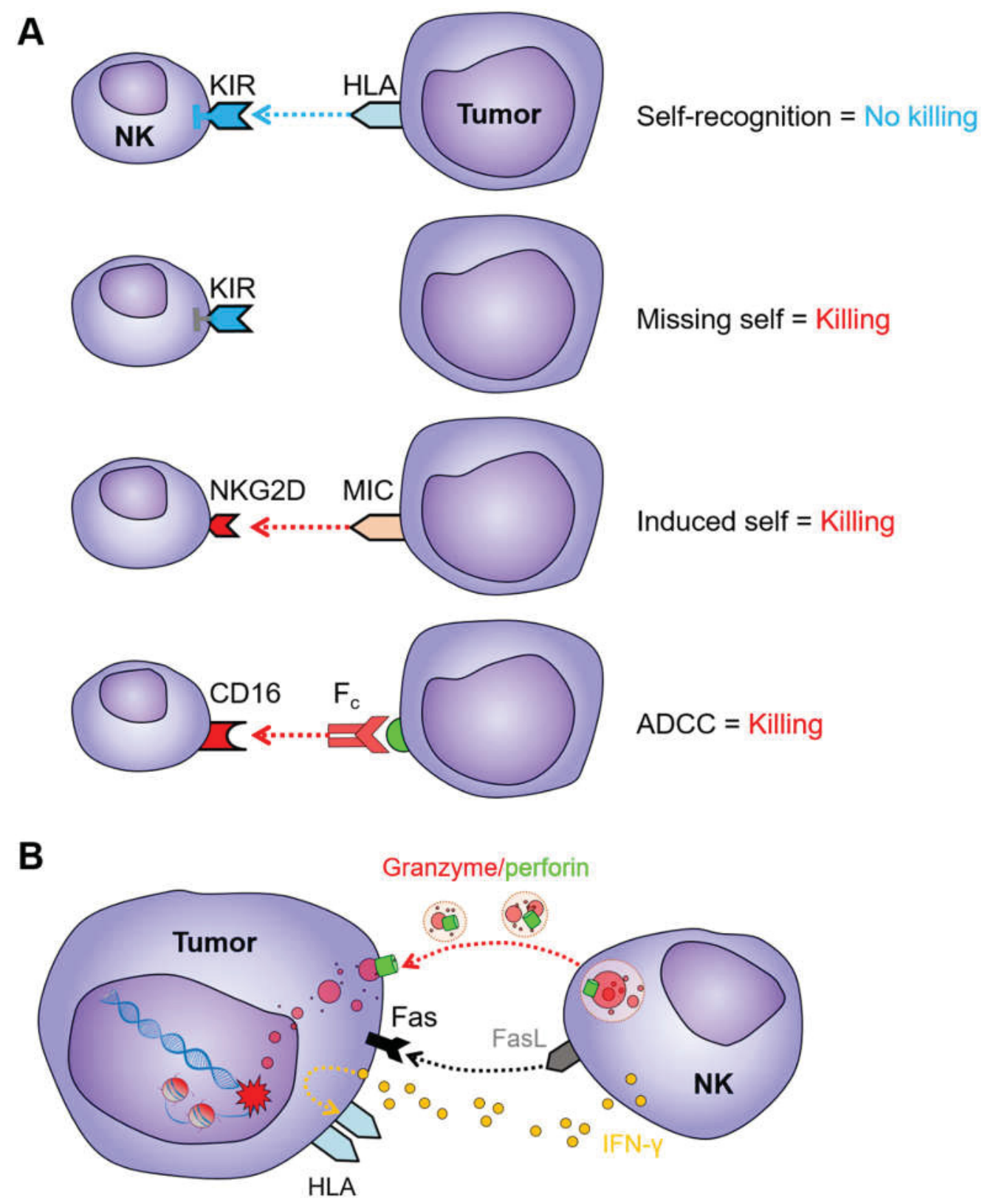

Figure 1-1. Mechanisms of target cell recognition and elimination by NK cells

Notes: (A) Receptor-ligand interactions are shown that determine the fate of target cells encountered by NK cells. (B) Mechanisms of NK cell killing. $\mathrm{F}_{\mathrm{c}}$, fragment, crystallizable; HLA, human leukocyte antigen; IFN- $\gamma$, interferon gamma; KIR, killer-cell immunoglobulin-like receptor; MIC, HLA class I chain-related; NK, natural killer.

Modified with permission from Springer Nature. Nguyen, R, et al. (2018). " The role of interleukin-2, all-trans retinoic acid, and natural killer cells: surveillance mechanisms in anti-GD2 antibody therapy in neuroblastoma." Cancer Immunol Immunother 67: 615-26. 
Table 1-1. Inhibitory and activating NK cell receptors and cognate ligands

\begin{tabular}{lll}
\hline NK cell receptor & Target cell ligand & Functional role \\
\hline KIR2DL2/3 (CD158b) & $\begin{array}{l}\text { HLA-C, group 1 } \\
\text { (Cw1, Cw3, Cw7, Cw8, Cw9, Cw10, }\end{array}$ & Inhibitory \\
& Cw12, Cw14, Cw16, B46, B73) & \\
KIR2DL1 (CD158a) & HLA-C, group 2 & Inhibitory \\
& (Cw2, Cw4, Cw5, Cw6, Cw15, Cw17, & \\
& Cw18) & \\
KIR3DL1 (CD158e1) & HLA-Bw4 & Inhibitory \\
& (B13, B27, B37, B44, B47, B38, B49, & \\
& B51, B52, B53, B57, B58, B59, B63, & \\
CD94/NKG2A & B77, A23, A24, A32) & \\
KIR3DL2 & HLA-E & Inhibitory \\
CD94/NKG2D & HLA-A3, A11 & Inhibitory \\
NKp44 & MICA, MICB, ULBP16 & Activating \\
NKp30 & PCNA & Activating \\
KIR2DS1/2 & BAT-3 & Activating \\
KIR2DL4 & HLA-C, group 1 and 2 & Activating \\
CD94/NKG2C & HLA-G & Activating \\
& HLA-E & Activating \\
\hline
\end{tabular}

Notes: HLA, human leukocyte antigen; KIR, killer-cell immunoglobulin-like receptors. 


\section{Natural killer cells for cancer-directed therapy}

Decades of research have improved our understanding of the interactions between the immune system and tumors. Consequently, numerous therapeutic advances for adult cancers have made their way into the clinic in the past 30 years, including interventions to modulate immune system-mediated ablation of tumor cells with antibodies that target tumor-specific antigens for ADCC and complement-dependent cytotoxicity [48]. Other strategies are geared towards chimeric antigen receptor (CAR) T cells [49] and immune check-point inhibitors to exploit $\mathrm{T}$ cell-mediated tumor cell ablation [50]. In addition, antibodies that abrogate cell receptor signaling (e.g., human epidermal growth factor receptor 2 [51]) or interfere with the tumor microenvironment (e.g., neutralizing antibodies against vascular endothelial growth factor [52]) are routinely used to provide therapeutic benefit.

During tumorigenesis and therapeutic selection pressure, tumor cells adopt strategies to evade effective host defense by the adaptive and innate immune system [53]. Mechanisms are summarized in Figure 1-2. To escape NK cell-mediated recognition and killing, classic mechanisms include the upregulation of MHC class I expression and downregulation of NK cell-activating ligands, such as MICA and MICB [54]. In addition, tumors can shed soluble MIC factors that disable NKG2D-mediated NK cell recognition, or they secrete inhibitory cytokines, such as transforming growth factor beta 1 (TGF- $\beta 1$ ), that directly inhibit NK cell function [55]. In the context of ADCC, downregulation of the target epitope can prevent antibody binding [56]. Newer studies have explored the role of CD155 in immune evasion. CD155, also known as polio virus receptor, is found on antigen-presenting cells and a variety of adult and pediatric cancers, including high-risk neuroblastoma, and is correlated with poor prognosis [57]. This molecule serves as a dual ligand for an activating (CD226) and inhibitory NK cell receptor (T cell immunoglobulin and immunoreceptor tyrosine-based inhibitory motif domain [TIGIT]) [58]. Despite dual recognition, the inhibitory signal from TIGIT overrides CD226 signaling and induces immune tolerance by blocking the PI3K/NFKB pathway [59]. This is because CD226 is downregulated and internalized after binding to CD155 [60], whereas TIGIT levels are maintained $[61,62]$. TIGIT-blocking antibodies can restore NK cell function and suppress tumor growth in preclinical models [58]. Therefore, the CD155-TIGIT-axis constitutes a possible future target for immunotherapeutic development. Aside from modulating the direct cellular interaction with NK cells, solid tumors may prohibit homing of NK cells to the tumor microenvironment and support the development of immunosuppressive tumor stroma $[63,64]$. Collectively, approaches to replace dysfunctional NK cells or restore NK cell function may prove essential to reverse immune evasion of cancer cells.

The first evidence for the therapeutic benefit of NK cells in cancer was established in a study of 57 adult patients with acute myeloid leukemia (AML), in which none of the 20 individuals who received haploidentical progenitor and stem cell transplantation from a KIR-HLA-mismatched donor experienced disease relapse [65]. Those findings have been replicated in preclinical experiments as well as in adult clinical trials $[66,67]$, without causing graft-versus-host disease. This has led to the development 
Figure 1-2. Mechanisms of tumor cell detection and evasion to escape NK cell recognition

Notes: (A) Interactions between NK cell receptors and tumor ligands are shown that lead to tumor cell recognition by NK cells. (B) Loss of specific receptor-ligand interaction can result in tumor immune evasion. $\mathrm{AB}$, antibody; GD2, disialoganglioside; HLA, human leukocyte antigen; IFN- $\gamma$, interferon gamma; KIR, killer-cell immunoglobulin-like receptors; MIC, HLA class I chain-related; NK, natural killer; TGF- $\beta$, transforming growth factor beta 1; TIGIT; T cell immunoglobulin and immunoreceptor tyrosine-based inhibitory motif domain; UL-BP16, UL16-binding proteins.

Sources: Groth A, Klöss S, Pogge von Strandmann E, et al (2011) Mechanisms of Tumor and Viral Immune Escape from Natural Killer Cell-Mediated Surveillance. J Innate Immun 3:344-354 [54]. https://doi.org/10.1159/000327014

Groh V, Wu J, Yee C, Spies T (2002) Tumour-derived soluble MIC ligands impair expression of NKG2D and T-cell activation. Nature 419:734-738 [55].

https://doi.org/10.1038/nature01112

Aldeghaither DS, Zahavi DJ, Murray JC, et al (2018) A Mechanism of Resistance to Antibody-Targeted Immune Attack. Cancer Immunol Res. [56]

https://doi.org/10.1158/2326-6066.CIR-18-0266

Gao J, Zheng Q, Xin N, et al (2017) CD155, an onco-immunologic molecule in human tumors. Cancer Sci 108:1934-1938 [57]. https://doi.org/10.1111/cas.13324

Zhang Q, Bi J, Zheng X, et al (2018) Blockade of the checkpoint receptor TIGIT prevents NK cell exhaustion and elicits potent anti-tumor immunity. Nat Immunol 19:723-732 [58]. https://doi.org/10.1038/s41590-018-0132-0

Stanietsky N, Simic H, Arapovic J, et al (2009) The interaction of TIGIT with PVR and PVRL2 inhibits human NK cell cytotoxicity. Proc Natl Acad Sci 106:17858-17863 [59]. https://doi.org/10.1073/pnas.0903474106

Carlsten M, Norell H, Bryceson YT, et al (2009) Primary human tumor cells expressing CD155 impair tumor targeting by down-regulating DNAM-1 on NK cells. J Immunol Baltim Md 1950 183:4921-4930 [60]. https://doi.org/10.4049/jimmunol.0901226 Bottino C, Castriconi R, Pende D, et al (2003) Identification of PVR (CD155) and Nectin-2 (CD112) as cell surface ligands for the human DNAM-1 (CD226) activating molecule. J Exp Med 198:557-567 [61]. https://doi.org/10.1084/jem.20030788 Li M, Xia P, Du Y, et al (2014) T-cell immunoglobulin and ITIM domain (TIGIT) receptor/poliovirus receptor (PVR) ligand engagement suppresses interferon- $\gamma$ production of natural killer cells via $\beta$-arrestin 2-mediated negative signaling. J Biol Chem 289:17647-17657 [62]. https://doi.org/10.1074/jbc.M114.572420 
A Immune surveillance

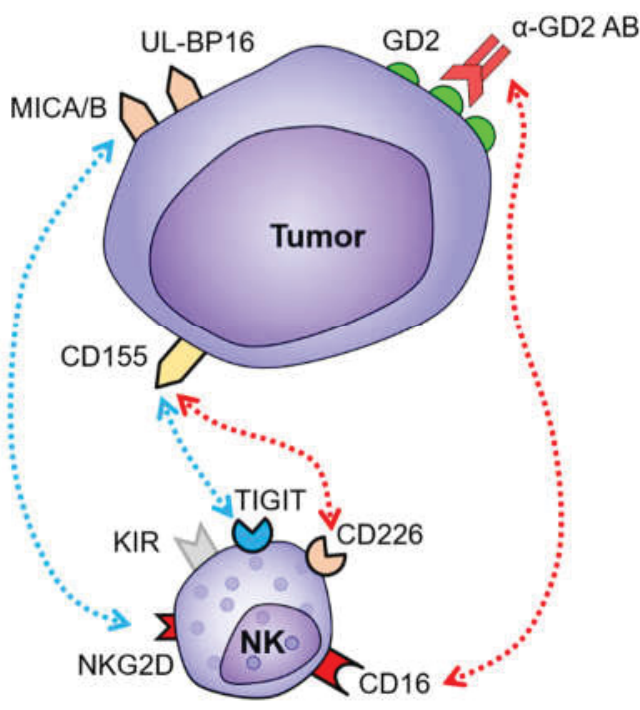

B Immune evasion

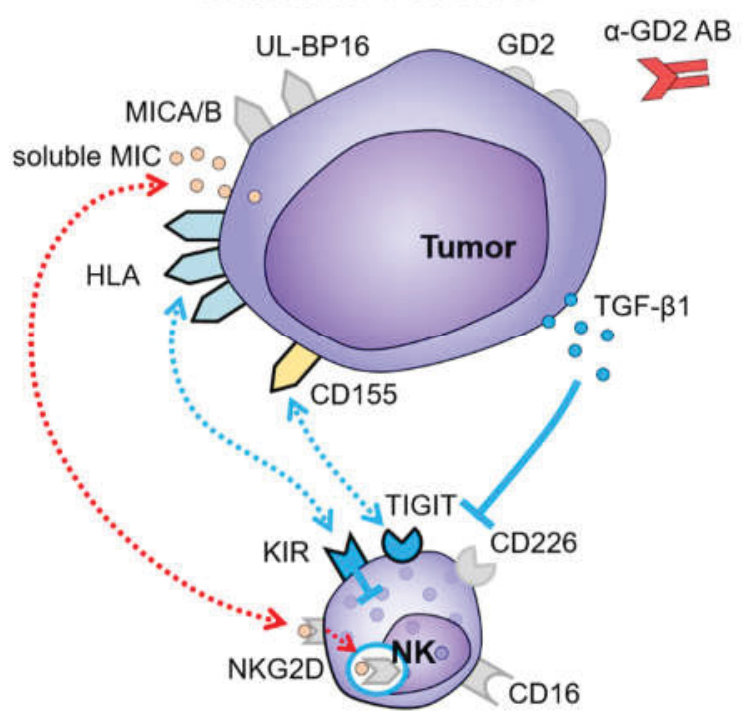


of two therapeutic approaches to harness the antileukemic effect of alloreactive NK cells for patients with AML. Patients may receive myeloablative chemotherapy followed by allogeneic hematopoietic progenitor and stem cell transplantation to induce permanent engraftment of donor KIR-HLA-mismatched NK cells [65]. Patients may alternatively receive lympho-depleting chemotherapy followed by adoptive transfer of purified NK cells from an alloreactive donor, resulting in homeostatic lymphocyte proliferation with transient donor NK cell expansion in vivo [68-70]. Because the allogeneic donor pool is limited, and because the donor selection process is very rigorous, there may not be an allogeneic KIR-HLA-mismatched donor available for all patients. However, due to the inheritance pattern of HLAs and KIRs, the likelihood of finding a KIR-HLA-mismatch is greatest among haploidentical donors. Using a haploidentical donor is ideal for pediatric patients because they are often accompanied by a parent who is commonly willing to undergo testing and donate NK cells; thus, allowing a timelier intervention.

Pediatric leukemias are the most common childhood malignancies, of which AML accounts for about $15-20 \%[71,72]$. Although the survival of children with AML has significantly improved over the past three decades and now approximates $90 \%$ with chemotherapy alone, one-third of children with AML experience disease relapse despite risk-adapted intensive multiagent chemotherapy with or without hematopoietic progenitor and stem cell transplantation [72]. These children have either intermediate or high-risk cytogenetic features; therefore, regimens are urgently needed to achieve higher cure rates while limiting therapy-related toxicities for this sub-group of patients. Based on previous clinical trials $[65,67]$, adoptive transfer of haploidentical NK cells were tested as a therapeutic option in a phase I trial in children with AML by Rubnitz et al. [69]. Ten patients (4 with low and 6 with intermediate-risk AML) participated in the study, all of whom were in first complete remission after four or five cycles of standard chemotherapy. Because previous clinical trials have demonstrated that a nonmyeloablative conditioning regimen facilitates NK cell persistence and expansion in vivo, children on this study received cyclophosphamide and fludarabine prior to NK cell infusion [68]. NK cells were isolated from the parental apheresis product in a two-step purification process consisting of a $\mathrm{CD}^{+} \mathrm{T}$ cell depletion followed by a CD $56^{+} \mathrm{NK}$ cell enrichment. Patients were infused with an average of $29 \times 10^{6} / \mathrm{kg}$ NK cells, and all participants tolerated the NK cell infusion without significant toxicity. In nine of 10 patients, KIR-HLA-mismatched NK cells expanded in vivo over the course of four weeks of observation. Additionally, all patients remained in complete remission at approximately 32 months, suggesting that adoptively transferred NK cells from a haploidentical KIR-HLA-mismatched donor may be beneficial as consolidative intervention to prolong the event-free survival in children with intermediate-risk AML [69]. Apart from these encouraging results, several other landmark trials have been published in adult and pediatric hematological malignancies and solid tumors [73-79]. These studies reported adoptive NK cell infusions to be safe and feasible but clinical benefit varied among the trials and was inferior in solid tumors than in leukemias [7379].

Although several successful immunotherapy trials have been conducted for pediatric leukemias and lymphomas, far fewer have been completed for pediatric solid 
tumors. To date, the most significant immunotherapy study for pediatric solid tumors investigated the efficacy of anti-disialoganglioside (GD2) antibody, IL-2, and granulocyte-macrophage colony-stimulating factor (GM-CSF) for neuroblastoma [80]. GD2 is a suitable target for monoclonal antibody therapy because it is abundantly expressed in most neuroblasts and restricted in normal cells (e.g., peripheral nerves) [81]. Because GD2 is a marker of mature neurons, anti-GD2 antibody is currently administered in combination with cis-retinoic acid that induces neuronal differentiation [82]. To enhance the anti-tumor effect, IL-2 and GM-CSF are added simultaneously to this regimen [83-85]. Neuroblastoma is the most common extracranial solid tumor of childhood with an incidence of 11 per million children under the age of 15 years [86]. It is the most common cancer in infants and toddlers, affecting patients at a median age of 17 months. Neuroblastoma is a developmental tumor that originates from primitive cells of the autonomic system. The heterogeneous biology of this tumor is reflected by disproportionate survival rates among patients. For example, among children with disseminated disease (stage 4), infants with stage 4S (special) may have spontaneous tumor regression and cure rates greater than $90 \%$ with very limited medical intervention [87]. However, infants with $M Y C N$ amplification and those aged 18 months or older with stage 4 disease, irrespective of $M Y C N$ status, have dismal survival rates and account for $15 \%$ of childhood cancer deaths $[88,89]$. These patients are classified as high-risk and receive multi-modal therapy, consisting of multi-agent chemotherapy, surgery, radiation therapy, tandem autologous bone marrow transplantation, and immunotherapy in combination with cis-retinoic acid $[80,90,91]$. The incorporation of immunotherapy into the standard of care for high-risk neuroblastoma occurred after Yu et al. reported in their landmark article that children who received immunotherapy with cis-retinoic acid had a 20\% 2-year event-free survival benefit compared to patients treated with cis-retinoic acid alone [80]. Children enrolled on this trial had completed induction chemotherapy, surgery, radiation therapy, and consolidation therapy before being randomized to the respective therapy arm for maintenance treatment. Despite this achievement, one third of children still experience treatment failure within the first two years of intervention and/or are unable to tolerate anti-GD2 antibody and IL 2-related side effects, respectively. Most common toxicities are antibody-induced neuropathy and systemic inflammatory response syndrome-like reactions due to IL-2 [80, 92]. The need for optimization of immunerelated therapeutic modalities is therefore crucial to overcome such toxicities, while maximizing disease response and survival. In an attempt to do so, clinicians have tried to engage immune surveillance and ADCC of NK cell killing by administering anti-GD2 antibody with an NK cell infusion from a haploidentical KIR-HLA-mismatched donor [93]. However, the cellular mechanisms of intervention-augmented ADCC are not fully understood, although they may directly affect treatment. For example, insight into the relative contribution of ADCC versus NK cell surveillance could determine whether and when adoptive transfer of NK cells is useful during treatment. In addition, although IL-2 activates NK cells and leads to more effective ADCC in preclinical testing [83], it remains unclear if continuous exposure to IL-2 is necessary to maintain this effect in immunotherapy for neuroblastoma. More knowledge in this regard may help define criteria for donor selection and help better understand the differential and combined effects of IL-2, retinoic acid, and anti-GD2 antibody in ADCC. 


\section{Preclinical Animal Models for Immunotherapy Testing}

In contrast to adult oncology, a relatively small number of children are diagnosed each year with pediatric malignancies and available to participate in clinical research [86]. But despite the highly effective infrastructure of clinical trials, conducting clinical studies in pediatric oncology has been inherently challenging because of low disease incidence, insufficient preclinical testing programs, and different perspectives on therapy success (i.e., striving for cure versus prolonged survival). The heterogeneous clinical behavior of the various disease entities adds yet another layer of complexity. In this context, preclinical animal models have become indispensable for advancing our understanding of the underlying biology and for drug development. However, due to genetic, molecular, and immunologic differences between mice and humans, these models have intrinsic limitations to reliably translate findings and predict outcomes in future human clinical trials [94]. For example, mouse models commonly replicate certain pathologic processes but fail to recapitulate the global spectrum of physiologic changes with therapeutic intervention [95]. Because negative findings often remain unreported, preclinical studies may overestimate actual response rates in humans by about $30 \%$ [ 96 , 97]. Consequentially, only a third of compounds tested in preclinical in vivo studies advance into human trials, of which as little as $8 \%$ of compounds successfully complete phase I testing [98]. Importantly, the interspecies differences may also mask the occurrence of potential side effect. For example, Melanoma-associated antigen 3 (MAGE-A3)/HLA-A1 T cell receptor (TCR) gene-engineered T cells caused cardiogenic shock and death in two adult patients with multiple myeloma and metastatic melanoma within two weeks of cellular infusion $[99,100]$. Autopsy revealed extensive cardiac Tcell infiltrate and diffuse myocyte necrosis, and death was attributed to cardiotoxicity in these cases. MAGE-A3 is not expressed in myocytes but detailed studies in the laboratory with contracting live myocytes in culture identified cross-reactivity of the MAGE-A3 TCR with the unrelated muscle-specific protein, Titin, that was not apparent in preclinical testing [101]. Collectively, understanding the limitations of preclinical animal modeling is critical to design preclinical experiments, interpret research findings, and translate discoveries into the clinic.

Mouse models for preclinical testing of cancer immunotherapies have complex variables that require thoughtful consideration during the process of experimental design. These variables are summarized in Figure 1-3 by the example of neuroblastoma. In general, murine models are divided into syngeneic and partially of fully xenogeneic transplantation models. When humanizing animals for experimental purposes, it is important to understand that the immunological response in mice may not occur in the same way in humans. For example, $C m v 1$, a susceptibility locus for cytomegalovirus infection, encodes the Ly49 family of proteins that are expressed on murine NK and NKT cells. They serve as inhibitory receptors for MHC I molecules but do not exist in humans [102]. Instead, KIRs fulfill an equivalent role but are structurally divergent from the Ly49 family. As such, the extracellular domain of KIRs contains immunoglobulin rather than C-type lectin domains. Similarly, the ligands for NKG2D in humans (MICA, MICB, and UL16-binding proteins) are replaced by $\mathrm{H}-60$ and Rae $1 \beta$ in mice $[43,102]$. The physiological significance of these interspecies differences in NK cell response to 


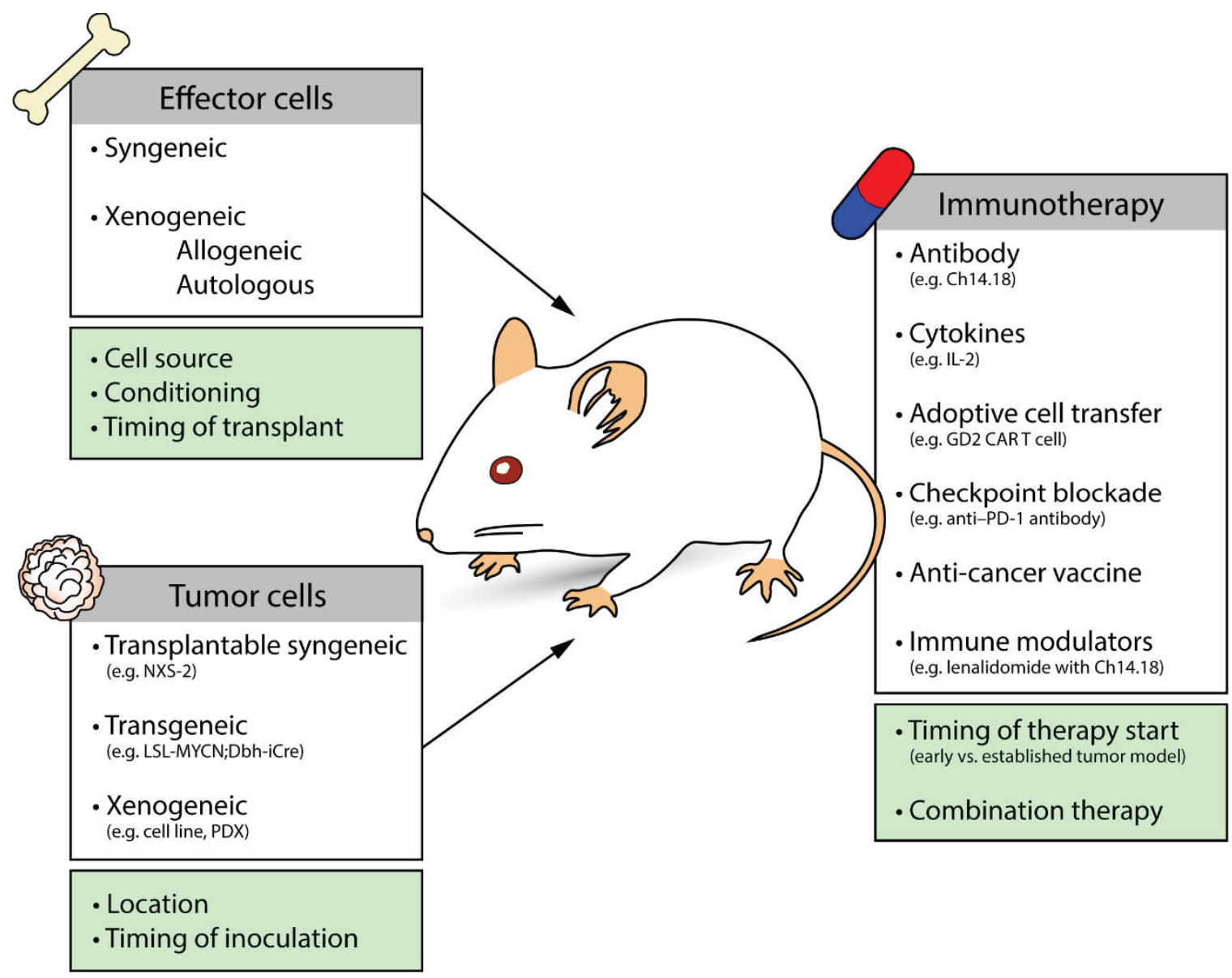

Figure 1-3. Common variables of mouse models of neuroblastoma for preclinical immunotherapy testing

Notes: Relevant variables for humanized tumor modeling and experimental design to test immunotherapies in vivo. GD2, disialoganglioside; IL-2, interleukin-2; PDX, patientderived xenograft; PD-1, programmed cell death-1. 
cytomegalovirus infection is not fully understood but may apply in similar ways to tumor surveillance.

Patient-derived xenografts (PDXs) have become a useful tool for preclinical testing because the tumor histology and microenvironment of engrafted PDXs more closely resemble that of human tumors than immortalized cell lines [103]. In addition, PDXs maintain genetic characteristics that are consistent with the genetic landscape of primary tumors. Pediatric PDX models have been used for drug discovery and development based on which successful clinical trials have been launched [104]. Integrating PDXs in mouse models of immunotherapy requires replacement of the murine immune system with human cells. This can be achieved by transplanting human hematopoietic progenitor and stem cells that will give rise to human effector cells [105, 106], or by adoptively transferring mature effector cells to humanize the animal [107]. Alternative ways to link the mouse and human system is by using a murine antibody that can recognize the epitope on the human tumor but allows the $\mathrm{F}_{\mathrm{c}}$ portion of the antibody to interact with murine effector cells. The sources of donor-unrelated CD $34^{+}$hematopoietic stem and progenitor cells are umbilical cord blood [108, 109], human bone marrow [110], human peripheral blood [111], or fetal liver [110]. For successful reconstitution, recipient mice must be immunocompromised and undergo a conditioning regimen to further lympho-deplete the animals and make space for the donor cells to engraft. Thus, transfer of human $\mathrm{CD}_{3} 4^{+}$cells into irradiated $\operatorname{Rag} 2^{\text {null }} / I l 2 R \gamma_{c}{ }^{\text {null }}(\mathrm{RG})$ or $N O D / s c i d / I l 2 R \gamma_{c}{ }^{\text {null }}$ (NSG) pups supports the basic development of human immune cells [111]. However, due to poor inter-species cross-reactivity of mouse and human cytokines, the composition of engrafted $\mathrm{CD} 45^{+}$immune cells usually lacks functional $\mathrm{NK}$ and $\mathrm{CD} 8^{+} \mathrm{T}$ cells and has insufficient amounts of granulocytes and monocytes/macrophages [112]. Researchers have tried to address this problem by creating transgenic mice that physiologically express human cytokines under the respective murine promotor. For example, transplanted $\mathrm{S}^{\mathrm{h} / \mathrm{m}} \mathrm{RG}-15^{\mathrm{h} / \mathrm{m}}$ mice that express human IL-15 and signal regulatory protein alpha (SIRPA) on an RG background have higher numbers of functional circulating and tissue-resident human $\mathrm{NK}$ and $\mathrm{CD}^{+} \mathrm{T}$ cells compared to NSG mice [112]. Importantly, these immune cells can perform tumor surveillance and mount an appropriate response to infection. The NSG-Tg(CMV-IL3,CSF2,KITLG) (SGM-3) mouse contains the three coinjected transgenes, $I L 3, C S F 2$, and $S C F$ under a cytomegalovirus promoter/enhancer sequence that render the hematopoietic progenitor and stem cells capable of engrafting without the need for irradiation and support hematopoiesis of innate immunity [113, 114]. Specifically, transplanted NSG-SGM-3 mice have proportionally higher amounts of $\mathrm{CD}_{3}{ }^{+}$myeloid cells than control NSG mice. Due to the unique cytokine environment, primary AML PDXs reportedly engraft in these animals as well. Lastly, $M-C S F^{\mathrm{h} / \mathrm{h}} I L$ 3/GM-CSF ${ }^{\mathrm{h} / \mathrm{h}} \operatorname{SIRP} A^{\mathrm{h} / \mathrm{m}} T P O^{\mathrm{h} / \mathrm{h}} \operatorname{Rag} 2^{\text {null }} / I l 2 R \gamma_{c}{ }^{\text {null }}$ (MISTRG) mice are a humanized mouse strain that foster a cytokine environment supportive of the development of human myeloid cells, functional NK cells, granulocytes, and dendritic cells [115].

Humanized mice that reconstituted their human immune system from CD34 hematopoietic progenitor and stem cells should not develop xenogeneic graft-versus-host disease (GVHD) in the absence of human thymus. Xenogeneic GVHD is a T cellmediated process, whereby human donor T cells attack murine cells due to HLA 
mismatch [116]. However, in xenograft models that lack human thymus, T cell education takes place in the mouse thymus, resulting in a T cell tolerant to mouse MHC but simultaneously incapable of mounting a human HLA-restricted immune response. This is problematic if the study of T cells in the context of tumor immunology is desired. To overcome this hurdle, human $\mathrm{CD}_{3} 4^{+}$cells were injected into NSG recipient mice and human fetal thymus and liver tissue were implanted under the kidney capsule of the animals [117]. These bone marrow-liver-thymus (BLT) mice supported the long-term reconstitution of an almost complete human immune system that includes immune competent T cells, B cells, NK cells, dendritic cells, and macrophages. However, only a few research programs have the privilege of utilizing BLT mice for their research due to the scarce availability of fetal donor tissue and the logistic and regulatory hurdles in obtaining such tissues.

NK cells that differentiate from human $\mathrm{CD} 34^{+}$cells in mice and express mature NK cell receptors can reject human tumor. This is because the allogeneic hematopoietic stem and progenitor cell donor may be KIR-HLA-mismatched to the tumor which can trigger a rejection-type immune response $[28,31]$. In this case, the use of effector cells from the same patient who donated the tumor constitutes an alternative way to engraft the mice with tumor-matched effector cells. Although peripheral blood mononuclear cells (PBMCs) are the easiest way to obtain such cells, differentiation and large-scale expansion of NK cells from CD34 ${ }^{+}$hematopoietic stem and progenitor cells may yield a larger amount of NK cells for preclinical testing, especially when dealing with pediatric patients who in some cases are too small to donate larger amounts of blood for experimental purposes $[118,119]$. In the future, further advances in science may allow the ex vivo generation of hematopoietic stem cells from renewable sources, such as tumor-matched patient-derived induced pluripotent stem cells. As for now though, the use of allogeneic donors will remain the mainstay for humanized transplantation and tumor models of immunotherapy.

The ability to predict the pharmacokinetics of a drug prior to first-in-human studies is critical to advance passed phase I clinical trials [98]. Therefore, preclinical pharmacokinetic studies are conducted in the process of drug discovery and development to ensure that patient-equivalent doses are tested in the laboratory and that respective response rates can be later reproduced in clinical trials with physiologically achievable drug concentrations. Preclinical testing of monoclonal antibodies in mice bears the risk of low antigen sequence homology, differing binding affinity to the neonatal $\mathrm{F}_{\mathrm{c}}$ receptor $\left(\mathrm{F}_{\mathrm{c}} \mathrm{Rn}\right)$, and lack of cross-reactivity with healthy tissue when compared to humans. This does not only affect the antibody clearance and efficacy but also potentially masks offtarget effects that later become apparent in humans [120-122]. Due to their large size and specific binding properties, monoclonal antibodies generally have a long half-life and are eliminated by means of two major routes [123]. The first order clearance occurs through endocytosis and resembles linear IgG clearance. In this process, the monoclonal antibody is endocytosed and undergoes intracellular proteolytic degradation via the ubiquitinproteasome pathway. $\mathrm{F}_{\mathrm{c}} \mathrm{Rn}$ prevents IgG and IgG-based antibodies from rapid intracellular catabolism and is the reason for the long half-life of these proteins. The second order pathway is non-linear and target-mediated. Following specific binding of 
the monoclonal antibody to its pharmacological target, the antibody-antigen complex is internalized for lysosomal degradation. Unlike the first order pathway, this route of catabolism saturates easily and is subject to antibody concentration and distribution, epitope expression, and rate of internalization. Lastly, it is important to acknowledge that the tissue and tumor distribution of monoclonal antibodies is very slow because it occurs through the process of diffusion. Altogether, preclinical testing of monoclonal antibody therapy is inherently complicated due to tissue-dependent clearance mechanisms. With the occurrence of novel $\mathrm{F}_{\mathrm{c}}$-fusion proteins, antibody-drug conjugates as well as bispecific antibodies, deeper knowledge of the pharmacokinetic properties of these proteins will allow more accurate translation of research findings.

\section{Specific Aims}

NK cells are known to play an important role in the natural defense against viral infections and elimination of transformed cells. Through complex interactions between NK cell receptors and target cell ligands, pathologic target cells are identified and rapidly eliminated. In pediatric oncology, NK cells have been used for for AML-directed therapy or to treat neuroblastoma. However, the cellular mechanisms in this process are not fully understood. Hence, the first objective of this dissertation work was to elucidate the cellular processes underlying ADCC in neuroblastoma. The second objective was to develop a humanized mouse model and validate its use for preclinical immunotherapy testing. The third objective aimed at relating preclinical findings to correlative biology studies in children with AML and neuroblastoma. I accomplished these objectives through the following specific aims:

\section{Objective 1: To elucidate the cellular mechanism underlying ADCC in neuroblastoma}

Sub aim 1-1: To distinguish the relative contribution of IL-2-augmented ADCC or NK cell-mediated natural cytotoxicity to neuroblastoma cell death

Sub aim 1-2: To compare the effect of IL-15 with IL-2 to enhance ADCC in neuroblastoma

\section{Objective 2: To develop a humanized mouse model and validate its use for preclinical immunotherapy testing}

Sub aim 2-1: To characterize human NK cells in a novel humanized tumor mouse model Sub aim 2-2: To describe the anti-tumor response to chemoimmunotherapy in a humanized tumor mouse model 
Objective 3: To relate preclinical findings to correlative biology studies in children with AML and neuroblastoma

Sub aim 3-1: To determine the efficacy of haploidentical KIR-HLA mismatched NK cells for consolidative therapy in a phase II study of children with intermediate-risk AML

Sub aim 3-2: To examine the quantitative and qualitative changes of NK cells in children with neuroblastoma before and during chemoimmunotherapy 


\section{CHAPTER 2. METHODOLOGY ${ }^{1}$}

\section{Tumor Cells}

Neuroblastoma cell lines were maintained in Roswell Park Memorial Institute 1640 medium (Lonza, Walkersville, MD; CHLA90, NBLS, SK-N-BE2, SK-N-JCI, SKN-MM), Dulbecco's modified eagle medium (Lonza; NB-1691, SK-N-AS, SK-N-FI), Eagle's minimum essential medium (EMEM; American Type Culture Collection [ATCC], Manassas, VA; IMR32, SK-N-SH), and EMEM/Kaighn's modification of Ham's F12 media (ATCC; SH-SY5Y) supplemented with 10\% heat-inactivated fetal bovine serum (Biowest, Kansas City, MO), $100 \mathrm{IU} / \mathrm{mL}$ penicillin, $100 \mu \mathrm{g} / \mathrm{mL}$ streptomycin, and $2 \mathrm{mM} \mathrm{L-glutamine}$ if base medium did not contain L-glutamine (all Gibco media, Grand Island, NY). The PDX lines SJNBL108_X, SJNBL047443_X, and SJNBL013761_X1 were grown orthotopically in CD1-Foxn $\overline{1}^{\text {nu }}$ immunodeficient mice (Charles River Laboratories, Wilmington, MA) and approved by the IACUC of St. Jude Children's Research Hospital. Palpable tumors were harvested and further processed into single-cell suspensions for ex vivo plating, as previously described.

\section{Animals}

CD1-Foxn $1^{\text {nu }}$ mice were used for propagation of PDXs and in vivo cytokine studies. NSG and MISTRG mice (both Jackson Laboratory, Bar Harbor, ME) served as animal strains for transplantation experiments with human effector and tumor cells. NSG mice were used for antibody saturation studies.

\section{NK Cell Preparation and Isolation}

NK cells from residual peripheral whole blood were collected from heparinized apheresis rings obtained from healthy de-identified blood donors. Each experiment in culture was performed with a new donor. Each mouse experiment that required NK cells for adoptive transfer was performed with one donor whose NK cells were frozen in small aliquots and thawed as needed. PBMCs were isolated via density-gradient centrifugation (GE Healthcare Ficoll-Paque PLUS, Marlborough, MA), and red blood cells were removed with lysis buffer (Qiagen, Hamburg, Germany). NK cells were isolated with RosetteSep Human NK Cell Enrichment Cocktail (Stem Cell Technologies, Cambridge,

\footnotetext{
${ }^{1}$ Portions of chapter from previously published article; final submission modified with permission of Springer Nature. Nguyen R, Houston J, Chan WK, et al. (2018) The role of interleukin-2, all-trans retinoic acid, and natural killer cells: surveillance mechanisms in anti-GD2 antibody therapy in neuroblastoma. Cancer Immunol Immunother, 67: 615-26 [134]. https://doi.org/10.1007/s00262-017-2108-6.

Portions of chapter from final submission modified with open access permission from Springer Nature. Nguyen R, Wu H, Pounds S, et al. (2019) A phase II clinical trial of adoptive transfer of haploidentical natural killer cells for consolidation therapy of pediatric acute myeloid leukemia. J. Immunother. Cancer. In press. Authors retain the copyright.
} 
MA) and human MACSxpress NK Cell Isolation Kit (Miltenyi Biotec, Bergisch Gladbach, Germany). IL-2 and IL-15 were provided by the Biological Resource Branch at the National Cancer Institute for preactivation of NK cells.

\section{Hematopoietic Stem and Progenitor Cells}

$\mathrm{CD} 34^{+}$hematopoietic stem and progenitor cells were isolated from commercially available apheresis products from granulocyte colony-stimulating factor (G-CSF) mobilized healthy adult donors using the CliniMACS system (Miltenyi) and provided by Dr. Mitchell J. Weiss at St. Jude Children's Research Hospital.

\section{Transplantation Procedures}

For bone marrow transplantation procedures in newborn mice, pups were exposed to sub-lethal irradiation (X-ray irradiation with Cesium irradiator) with $1 \times 100 \mathrm{cGy}$ (NSG) or $1 \times 150 \mathrm{cGy}(\mathrm{MISTRG})$ within the first two days of life and subsequently, $100,000 \mathrm{CD}^{+} 4^{+}$cells in $10 \mu \mathrm{l}$ of phosphate-buffered saline (PBS) were injected into the liver with a 30-gauge needle (Hamilton Company, Reno, NV). For bone marrow transplantation in adult mice, 4-6-week-old animals underwent irradiation with $1 \times 100$ cGy (NSG) or $1 \times 250$ cGy (MISTRG). Then, 100,000 CD34 ${ }^{+}$cells in $200 \mu$ of PBS were injected intraperitoneally with a 27 -gauge hubless tuberculin syringe (Becton Dickinson, Franklin Lakes, NJ). Transplanted mice were bled 7-9 weeks later and the levels of human $\mathrm{CD} 45^{+}$cells was measured in the peripheral blood by flow cytometry and calculated as engraftment $(\%)=\left(\mathrm{CD} 45^{+}\right.$cells $/\left[\mathrm{CD} 45^{+}\right.$cells $+\mathrm{Cd} 45^{+}$cells $\left.]\right)$. PDX was resuspended as a single-cell solution in Matrigel (Corning Inc., Corning, NY) at a concentration of 10,000 cells/ $\mu 1$. Under ultrasound guidance, mice were injected with 10 $\mu l$ in the paraadrenal region at 5-6 weeks of life. Animals who underwent adult bone marrow transplantation had a 72-hour recovery period in between procedures. Male or female animals with tumor measuring $\geq 10 \mathrm{~mm}^{3}$ by ultrasound were separated randomly into the experimental groups. All animal experimentations were performed in compliance with the IACUC of St. Jude Children's Research Hospital.

\section{Monoclonal Therapeutic Antibodies}

The anti-GD2 antibody Hu14.18K322A was provided to St. Jude Children's Research Hospital and Children's GMP, LLC (Memphis, TN) by Merck Serono (Darmstadt, Germany) and was manufactured by Children's GMP, LLC. Hu14.18K322A was used in all in vivo and in vitro experiments with human neuroblastoma and NK cells. For experiments with murine effector cells and human neuroblastoma cells, the monoclonal antibody 14.G2a provided by the Biological Resource Branch at the National Cancer Institute was used instead. The anti-HLA antibody W6/32 (Biolegend, San Diego, $\mathrm{CA}$ ) recognizes a monomorphic epitope on the $45 \mathrm{kDa}$ polypeptide products of HLA-A, $\mathrm{B}$, and $-\mathrm{C}$ and was used at a concentration of $1.5 \mu \mathrm{g} / \mathrm{mL}$ to block HLA. 


\section{ADCC and NK Cytotoxicity Assays and Confluence Analysis}

For the ADCC and NK cytotoxicity assays, neuroblastoma cells were cultured on 96-well flat-bottom plates (Corning Inc.) at $37^{\circ}$ in 5\% CO2 incubators. The effector cellto-target cell $(\mathrm{E}: \mathrm{T})$ ratio was determined by performing a semiautomated nuclear cell count after 4',6-diamidino-2-phenylindole (DAPI) stain with Fiji (Curtis Rueden and Image J, Madison, WI) and manual count of neuroblastoma cells. To induce ADCC, different dilutions of Hu14.18K322A were added to the culture wells one hour prior to co-incubating effector cells with tumor cells. NK cytotoxicity assays were performed with effector cells and target tumor cells using the conditions and quantification described above for the ADCC assay; however, no Hu14.18K322A was added. CalceinAM (Thermo Fisher, Waltham, MA) was used at a concentration of $2 \mathrm{nM}$ to perform live-cell staining. Images of calcein-AM-stained cells were acquired with a C2 Nikon confocal microscope (Nikon, Melville, NY). Images were further processed with the Fiji plugin Trainable Weka Segmentation that combines machine-learning algorithms with selected image features to conduct pixel-based segmentations. Segmented areas were quantified and represented confluence. Specific lysis was calculated with the following formula: specific lysis $(\%)=(1-($ confluence untreated - confluence treated $) /$ confluence untreated) $\times 100$. For ADCC assays with PDXs, the CellTiter-Glo luminescent cell viability assay (Promega, Madison, WI) was used to quantify specific lysis. To determine the NK cell-mediated cytotoxicity of peripheral blood NK cells from patients, NK cells were co-cultured with yellow fluorescent protein-expressing K562 for four hours. The E:T ratio was 5-1. At the end of the incubation time, residual tumor cells were quantitated by flow cytometry and results were normalized with particles for absolute cell counting (Spherotech, Lake Forest, IL).

\section{CD107a Degranulation Assay}

NK cells were incubated with neuroblastoma cells, in addition to anti-CD107a antibody (clone H4A3, Biolegend). After one hour, GolgiStop solution (BD Biosciences, San Jose, CA) was added, and cells were incubated for another three hours before staining for flow cytometry analysis.

\section{Sphere Formation and Infiltration Assay}

Yellow fluorescent protein-expressing PDX were processed into a single-cell suspension and resuspended in Dulbecco's modified eagle medium (Lonza) supplemented with 10\% heat-inactivated fetal bovine serum (Biowest, Kansas City, MO), $100 \mathrm{IU} / \mathrm{mL}$ penicillin, $100 \mu \mathrm{g} / \mathrm{mL}$ streptomycin, and $10 \mu \mathrm{M}$ rho kinase pathway inhibitor Y-27632 (Stemcell Technologies). Tumor cells were plated at a density of 50,000 cells per well into a 96-well low-attachment plate, spun down at $100 \mathrm{RCF}$ for five minutes, and maintained at $37^{\circ}$ in 5\% CO2 incubators. On day 3 after plating, when spheroid formation had occurred, single spheres were transferred into a 24 -well plate and cultured for another 24-48 hours before using them for infiltration assays. To determine NK cell 
infiltration into the spheres, NK cells were first labeled with CellTracker ${ }^{\mathrm{TM}}$ Orange (Thermo Fisher Scientific) as directed by the manufacturer's instructions. Then, 100,000 NK cells were co-incubated with spheres for 12 hours. Spheroids were washed in PBS, fixed in paraformaldehyde (4\% in PBS) for two hours, washed again, and placed in 30\% sucrose in PBS before proceeding with cryosectioning. Cryosections were stained with DAPI and imaged with a C2 Nikon confocal microscope (Nikon). Tumor-infiltrating NK cells were counted manually on each section and results were normalized by the sectioned area of tumor.

\section{Differentiation Assay}

To induce neuronal differentiation, neuroblastoma cells were incubated with 10 $\mu \mathrm{M}$ all-trans retinoic acid (ATRA; Sigma Aldrich, St. Louis, MO) for $72 \mathrm{~h}$.

\section{Bulk RNA Sequencing}

Trizol (Invitrogen, Carlsbad, CA) RNA extraction was used to isolate total RNA, which was prepared by TruSeq and sequenced with an Illumina HiSeq 2500 (San Diego, CA). Resultant stranded paired-end 100-bp sequences were mapped to the hg19 reference genome with the STRONGARM pipeline and counted with HTSeq (Simon Anders and EMBL Heidelberg, Germany). Fragments per kilobase of transcript per million mapped reads were calculated and then $\log 2$-transformed after the addition of a small variance stabilizing bias of 2 and $\log \mathrm{FC}$ calculated for the ATRA treatment within each cell line. Differential gene expression was defined as fold change of $>2$ or $<0.5$.

\section{Single-Cell RNA Sequencing Data Processing and Analysis}

Approximately 10,000 FACS-purified $\mathrm{CD} 45^{+} \mathrm{CD} 56^{+} \mathrm{CD} 3^{-} \mathrm{NK}$ cells $(>90 \%$ viability) were combined into droplets with barcoded beads by using the Chromium single-cell gene expression platform (10x Genomics) with the Chromium controller. The barcoded libraries were generated according to manufacturer specifications. The individual samples were sequenced to an average depth of 250,000,000 reads with an Illumina Hiseq sequencer.

CellRanger software (v1.3.1) was used to align raw FASTQ files with the GRch38 genome (http://cf.10xgenomics.com/supp/cell-exp/refdata-cellranger-GRCh381.2.0.tar.gz) and to generate a raw digital gene expression matrix (unique molecular identifier counts/gene per cell). For comparison among MISTRG, patient, and healthy control NK cells, the respective samples were pooled with the CellRanger aggregate function and analyzed individually. The data were filtered in R (https://www.Rproject.org/) with Seurat v3 [124]. The initial quality control step removed genes that were detected in less than 50 cells (unique molecular identifier count $>0$ ). The cells were filtered out if they contained a small library size ( $<200$ gene counts $)$ with a 
mitochondrial-to-genome transcript ratio of greater than 0.5. Cells with a high gene count $(>2,500)$ were also removed. Cell cycle or mitochondrial regression was not performed. Data were normalized and scaled before further analysis. Unsupervised hierarchical clustering was performed with the FindClusters function by using Seurat v3. Principal component analysis was used to reduce the number of dimensions, and the number of used components was selected based on the elbow of a scree plot. T-distributed stochastic neighbor embedding of the principal components was applied for two-dimensional visualization. Upregulated markers were identified with the FindMarkers function in Seurat with an adjusted $P$ value $<0.05$. Known NK cell subsets were assigned to the respective cluster according to previously published transcriptional signatures [125]. Other contaminating cell types were identified with MS4A7 (i.e., macrophages) and CD79A (i.e., B cells). Functional annotations of the most differentially expressed genes were retrieved from public databases (Uniprot and Genecards). Gene Ontology enrichment analysis was performed with the top 20 upregulated genes for each cluster. Scores were calculated with a threshold of significance of 0.05 and plotted as $-\log 10$ of the $P$ value according to Benjamini and Hochberg correction (http://geneontology.org/).

\section{HLA Genotyping and Definition of KIR-HLA Mismatch}

All neuroblastoma cell lines used in this work were HLA-genotyped and expression was confirmed by flow cytometry. CD158a (2DL1) was defined to be specific for HLA-C allotypes with lysine at position 80 (HLA-CLys80), CD158b1/b2 (2DL2/2DL3) to be specific for B*4601 and HLA-C allotypes with asparagine at position 80 (HLA-CAsn80), and CD158e1 (3DL1) to be specific for HLA-B allotypes expressing the Bw4 epitope (HLA-Bw4).

\section{Flow Cytometry and Fluorescence-Activated Cell Sorting (FACS)}

Expression of KIRs was determined by flow cytometry. The following antibodies were used: CD158a (clone 143211; R\&D systems, Minneapolis, MN), CD158b1/2 (DX27; Biolegend), CD158e1 (DX9; Biolegend), and CD159a (REA110; Miltenyi Biotec). For analysis, $\mathrm{CD}^{-}$(UCHT1) and CD56 ${ }^{+} \mathrm{NK}$ cells (NCAM16.2; BD) were gated. KIR phenotype analysis was conducted with WinList, v9.0 (Verity Software House, Topsham, ME). For analysis of expression of natural cytotoxicity receptors and NKG2D, the following antibodies were used: NKp30 (p30-15), NKp44 (P44-8), NKp46 (9E2), and NK cell G2D (1D11; all Biolegend). HLA status was recognized by W6/32 (Biolegend). For phenotypic analysis of murine immune cells, the following antibodies were used: Cd11c (N418), Cd49b (DX5), Ly-6C (1A8), Ly-6G (HK1.4), MHC II (M5/114.15.2; all Biolegend), B220 (RA3-6B2; Tonbo Bioscience, San Diego, CA), Cd45 (30-F11), Cd11b (M1/70), Cd27 (LG 3A10; all BD Biosciences, San Jose, CA). For blocking, the purified human FcR binding inhibitor (Thermo Fisher) and anti-mouse CD16/CD32 (2.4G2; Tonbo Bioscience, San Diego, CA) were used. 


\section{Preclinical Immunotherapy Testing In Vivo}

All in vivo testing was performed with tumor-bearing mice whose tumor volume measured $\geq 10 \mathrm{~mm}^{3}$ by ultrasound (established tumor model). Animals were then randomized to respective therapy groups. Across all experiments, chemotherapy was given during week 1 of each cycle and consisted of daily intraperitoneal irinotecan (1.6 $\mathrm{mg} / \mathrm{kg})$ and oral temozolomide $(16.5 \mathrm{mg} / \mathrm{kg})$ for five days. The immunotherapy regimen in experiments utilizing MISTRG mice after hematopoietic progenitor cell transplantation or adoptive NK cell transfer was administered on week 2 of each cycle and comprised of daily Hu14.18K322A (100 $\mu \mathrm{g}$ per animal per day) via tail vein and daily intraperitoneal recombinant human IL-2 (10,000 IU per animal per day) for five days.

To test the efficacy of IL-15/IL-15 receptor $\alpha$-subunit (IL-15R $\alpha$ ) complex in CD1-Foxn $1^{\mathrm{nu}}$ mice, animals were randomized to four treatment groups: chemotherapy alone (group 1); chemotherapy followed by anti-GD2 antibody and GM-CSF (group 2); chemotherapy followed by anti-GD2 antibody, GM-CSF, and IL-2 (group 3); chemotherapy followed by anti-GD2 antibody, GM-CSF, and IL-15/IL-15R $\alpha$-complex (group 4). Precomplexing of IL-15/IL-15R $\alpha$ was performed with recombinant mouse IL15 (Peprotech, Rocky Hill, NJ) and mouse IL-15R $\alpha$ Fc chimera (R\&D) as such that 2.5 ug of IL-15 and $12 \mathrm{ug}$ of IL-15R $\alpha$ were incubated at room temperature for $30 \mathrm{~min}$ to form the complex. The IL-15/IL-15R $\alpha$-complex was administered intraperitoneally to group 4 mice on day 1 of week 2 of treatment together with $50 \mathrm{ug} / \mathrm{kg} /$ day of intraperitoneal GMCSF. Mice that were randomized to group 3 were given daily 10,000 IU of recombinant mouse IL-2 (Peprotech) intraperitoneally and daily GM-CSF for five days of week 2. Animals assigned to group 2 received daily GM-CSF. All animals in group 2, 3, and 4 received 100 ug of $14 \mathrm{G} 2 \mathrm{a}$ anti-GD2 antibody on day 2-5 of week 2 . Each therapy cycle lasted for three weeks and a total of two cycles were administered to animals.

\section{GD2 Detection in Tumor Tissue}

NSG animals were injected via tail vein with $4 \mu \mathrm{g} / \mathrm{kg}$ Hu14.18K322A. Animals were sacrificed at pre-determined time points and perfused with PBS. Tumor tissue was removed and mechanically processed through a 40-micron cell strainer (Becton Dickson). The single-cell suspension was counterstained with an anti-human $\operatorname{IgG~} \mathrm{F}_{\mathrm{c}}$ antibody (HP6017; Biolegend) and then analyzed by flowcytometry.

\section{Patient Enrollment Criteria for NB2012 Study}

All patients with neuroblastoma that were examined in this work were enrolled in an institutional phase II trial (NCT01857934). The study was approved by the institutional review board, and informed consent was obtained from parents or guardians, and assent from the patients, as appropriate. 


\section{NK Cell Collection and Treatment Regimen of the NB2012 Study}

Children with newly-diagnosed, high-risk neuroblastoma were enrolled to receive induction chemotherapy identical to that described in the ANBL0532 trial combined with Hu14.18K322A (40 mg/m²; days 2-5), subcutaneous IL-2 $\left(1 \times 10^{6}\right.$ units $/ \mathrm{m}^{2}$; days 6,8 , $10,12,14$, and 16), and daily subcutaneous GM-CSF $\left(250 \mu \mathrm{g} / \mathrm{m}^{2}\right.$; day 7 until neutrophil count $\geq 2,000 \mathrm{~mm} 3$ ). The consolidation regimen consisted of myeloablative busulfan and melphalan, followed by autologous hematopoietic progenitor cell infusion. During consolidation, consenting patients received haploidentical $\mathrm{CD}^{2} 6^{+} \mathrm{CD} 3^{-} \mathrm{NK}$ cells purified in a two-step procedure with the CliniMACS system (Miltenyi Biotec) as preciously described. NK cells were infused any time between day 2 to 5 after autologous hematopoietic progenitor cell infusion and four additional doses of immunotherapy with daily intravenous GM-CSF $(250 \mu \mathrm{g} / \mathrm{m} 2$; beginning day 10 after NK cell infusion until neutrophil count $\geq 2,000 \mathrm{~mm} 3$ ). Maintenance therapy was administered as described in the ANBL0032 trial with Hu14.18K322A substituting ch14.18. Patients were stratified according to the International Criteria for Neuroblastoma Response into responders (i.e., complete, very good partial, and partial response) and those with stable disease at the time of completion of two induction therapy courses. There were no patients with progressive disease.

\section{Correlative Biology Studies on the NB2012 Study}

Peripheral blood NK cell phenotyping was performed on days 0,7 , and 21 of induction courses 1, 3, and 4; 7 and 21 days after haploidentical NK cell infusion during consolidation; and before maintenance therapy courses 1, 3, and 6 by flow cytometric analyses of cell surface receptor expression. Prior and following each course of therapy, NK cells were isolated from the peripheral blood as described above and cultured for 12 18 hours in media alone or supplemented with IL-2 (50 U/mL) or IL-15 (10 or $1 \mathrm{ng} / \mathrm{mL})$. Cultured NK cells were then subjected to a K562 kill assay to determine NK cellmediated cytotoxicity.

\section{Patient and Donor Enrollment Criteria for AML-NK Trial}

Children with intermediate-risk AML who were in first complete remission after completion of four or five courses of chemotherapy as part of the randomized controlled phase III clinical trial (NCT00703820) and who had a KIR--HLA-mismatched parent were eligible for adoptive transfer of haploidentical NK cells. No other criteria were considered for eligibility. Intermediate risk was defined by (1) presence of core binding factor leukemia with minimal residual disease of $\geq 0.1 \%$ but $<5 \%$ on Day 22 of therapy or rising levels of fusion transcript, (2) presence of FLT3-ITD fusion and negative minimal residual disease on Day 22 of therapy, or (3) absence of low-risk or high-risk features as previously defined. HLA and KIR genotyping, was performed for every patient and donor in a laboratory at St. Jude Children's Research Hospital. Potential donors underwent clearance procedures to determine eligibility. The study was approved 
by the institutional review board of St. Jude Children's Research Hospital, and informed consent was obtained from parents or guardians, and assent from the patients, as appropriate. Patients were monitored for 4five days after NK cell infusion for absolute neutrophil ( $\geq 500$ cells $/ \mu \mathrm{L}$ and rising) and platelet count recovery $(\geq 20,000$ cells $/ \mu \mathrm{L}$ and rising), graft-versus-host disease, and adverse events $\geq$ grade 3 . Patients with intermediate-risk AML who did not receive NK therapy but had completed at least four courses of chemotherapy as part of the randomized controlled phase III clinical trial (NCT00703820) were used as control cohort for survival analysis.

\section{NK Cell Collection and Treatment Regimen of the AML-NK Trial}

Patients with rising absolute neutrophil counts $\geq 300 / \mu \mathrm{L}$ and platelet counts $\geq 30,000 / \mu \mathrm{L}$ received the following conditioning regimen: Cyclophosphamide (60 $\mathrm{mg} / \mathrm{kg}$ ) was intravenously (IV) administered on Day -7 , and fludarabine $\left(25 \mathrm{mg} / \mathrm{m}^{2}\right.$ per day) was IV administered on Days -6 through -2 . Patients received interleukin-2 $(1 \times$ 106 units $/ \mathrm{m}^{2}$ ) subcutaneously on Days $-1,1,3,5,7$, and 9. Donors underwent apheresis on Day -1 , and mononuclear cells were purified in a two-step procedure for $\mathrm{CD}^{+} 6^{+} / \mathrm{CD}^{-}$ NK cells with the CliniMACS system (Miltenyi Biotec) as preciously described, allowing for a $\mathrm{CD}^{-} 6^{-} / \mathrm{CD}^{+}$cell dose of $<0.05 \times 10^{6}$ cells $/ \mathrm{kg}$. Purified, unmanipulated NK cells were infused on Day 0 at a desired dose of $>2 \times 10^{6} \mathrm{CD}^{2} 6^{+} / \mathrm{CD}^{-} \mathrm{NK}$ cells $/ \mathrm{kg}$ recipient body weight.

\section{Correlative Biology Studies on the AML-NK Trial}

NK cell chimerism and phenotyping from peripheral blood were performed on Days 7, 14, 21, and 28 after NK cell infusion. While blood for phenotype analysis was collected on time, there was up to a 48-hour deviation from these time points when blood was sampled for NK cell chimerism studies due to clinical care considerations (e.g. maximum blood draw limit). Chimerism studies of NK cells purified by fluorescenceactivated cell sorting were performed by standard variable number tandem repeats techniques. NK cell phenotyping was determined by flow cytometric measurement of cell surface receptor expression and antibodies are listed above. Alloreactive NK cells were defined as the fraction of $\mathrm{CD}^{2} 6^{+} / \mathrm{CD}^{-}$cells that expressed the HLA-mismatched KIR by flow cytometry analysis but was negative for the NKG2A and KIRs corresponding with patient expressed HLAs. A rise in donor NK cell chimerism or KIR-HLA mismatched NK cell number after Day 7 was defined as NK cell expansion. 


\section{Statistical Analysis}

\section{Objective 1}

Student's paired t test, one-way ANOVA, and multiple regression analysis were used to calculate statistical significance between groups (defined as $\mathrm{P} \leq 0.05$ ). All analyses were conducted with JMP Pro 12 (SAS Institute Inc., Cary, NC).

\section{Objective 2}

I applied D'Agostino-Pearson omnibus and Shapiro-Wilk normality tests to test all data for normal distribution of variables. I compared normally distributed groups with the two-tailed Student's t test for paired and unpaired data (two groups) and the one-way analysis of variance and Tukey's multiple comparison for post-hoc analysis (multiple groups). If data was not normally distributed, I applied the unpaired Mann-Whitney test (two groups) and the unpaired Kruskal-Wallis test with Dunn's multiple comparison (multiple groups). Parametric Pearson test was used for correlation analysis. All statistical analyses were performed with GraphPad Prism 8. A $P$ value $<0.05$ was considered statistically significant.

\section{Objective 3 (AML study)}

Summary statistics were used to report clinical information. Both trend effects and time points difference in mean white blood cell and NK cell counts over time were examined by using linear mixed-effects models. Spearman correlation was performed to test the relation between NK cell chimerisms and NK cell dose. In analysis of event-free survival, an event was defined as relapse or death. Survival analysis was performed by comparing patients who received NK cell therapy with those who also had intermediaterisk AML and did not receive NK therapy but had completed at least four courses of chemotherapy in the AML08 clinical trial (NCT00703820). A linear Cox hazard proportional regression model was applied to logarithmically transformed NK cell receptor expression and NK cell counts to assess their association with survival. Data were analyzed using R (https://www.R-project.org/).

\section{Objective 3 (neuroblastoma study)}

Patient characteristics were summarized by descriptive statistics (mean $\pm \mathrm{SD}$, median [range]). The distribution of cell counts and cell surface receptor counts and percentages over time was illustrated by summary statistics and box-and-whisker plots using a logarithmic scale. Zeros were assigned a value of 10 prior to logarithmic transformation for improved visual display based on common practice; however, statistical calculations were performed on untransformed data. Wilcoxon's signed rank 
test was used for pairwise comparison of NK cell counts. Two-sample t-tests were used to determine differences for remaining continuous variables between patient subgroups. The association between continuous variables were assessed by the Pearson correlation coefficient. Statistical analyses were conducted by using SAS version 9.4 (SAS Institute, Cary, NC). A two-sided significance level of $P<0.05$ was used for all statistical tests. 


\section{CHAPTER 3. RESULTS ${ }^{2}$}

\section{Objective 1}

\section{Development and validation of an assay for NK cell-mediated cytotoxicity against neuroblastoma cells}

Most assays of NK cell-mediated killing of tumor cells use purified peripheral blood NK cells and target tumor cells in suspension $[126,127]$. The advantage of this approach is that flow cytometry-based quantification of target-cell viability and studies of NK-cell properties can be conducted at the same time. However, these methods are suboptimal for neuroblastoma cells because they are adherent, and their dissociation from culture plates can lead to cell death. Therefore, an assay was developed in which purified NK cells were added to neuroblastoma cells in a 96-well plate, and NK cell-mediated cytotoxicity was analyzed in situ. Following co-incubation of effector and target cells, viable neuroblastoma cells were stained with calcein-AM and quantified by semiautomated confluence analysis. Residual NK cells were excluded from the analysis by their smaller size. The assay is schematically depicted in Figure 3-1.

Nine neuroblastoma cell lines were selected that span the spectrum of GD2 and HLA expression. HLA expression of neuroblastoma cells was studied by flow cytometry and compared to the HLA-negative cell line K562 and peripheral blood to classify HLA expression as low, intermediate, or normal. Purified peripheral blood NK cells from healthy donors were used as effector cells, and the expression of CD158a, CD158b, CD158e1, NKp30, NKp44, NKp46, and NKG2D was determined by flow cytometry. IL2 at a concentration of $50 \mathrm{IU} / \mathrm{mL}$ was used for NK cell activation. ADCC was induced by Hu14.18K322A, which is $98 \%$ humanized and contains an alanine substitution at lysine 322 to decrease allodynia and complement-dependent cytotoxicity [128]. Concentrations of $10 \mu \mathrm{g} / \mathrm{mL}$ of Hu14.18K322 were used as described in previous studies [129]. The incubation times and E:T ratios were optimized across all neuroblastoma cell lines for ADCC, which were 12 hours and 5 to 1 , respectively because it was the shortest time to achieve statistically significant differences in normalized relative cell killing across multiple neuroblastoma cell lines as shown in Figure 3-1.

Live-cell imaging was used as an independent method for assay validation. In a co-culture experiment, the specific lysis by allogeneic NK cells against two

\footnotetext{
${ }^{2}$ Portions of chapter from previously published article; final submission modified with permission of Springer Nature. Nguyen R, Houston J, Chan WK, et al. (2018) The role of interleukin-2, all-trans retinoic acid, and natural killer cells: surveillance mechanisms in anti-GD2 antibody therapy in neuroblastoma. Cancer Immunol Immunother, 67: 615-26 [134]. https://doi.org/10.1007/s00262-017-2108-6.

Portions of chapter from final submission modified with open access permission from Springer Nature. Nguyen R, Wu H, Pounds S, et al. (2019) A phase II clinical trial of adoptive transfer of haploidentical natural killer cells for consolidation therapy of pediatric acute myeloid leukemia. J. Immunother. Cancer. In press. Authors retain the copyright.
} 

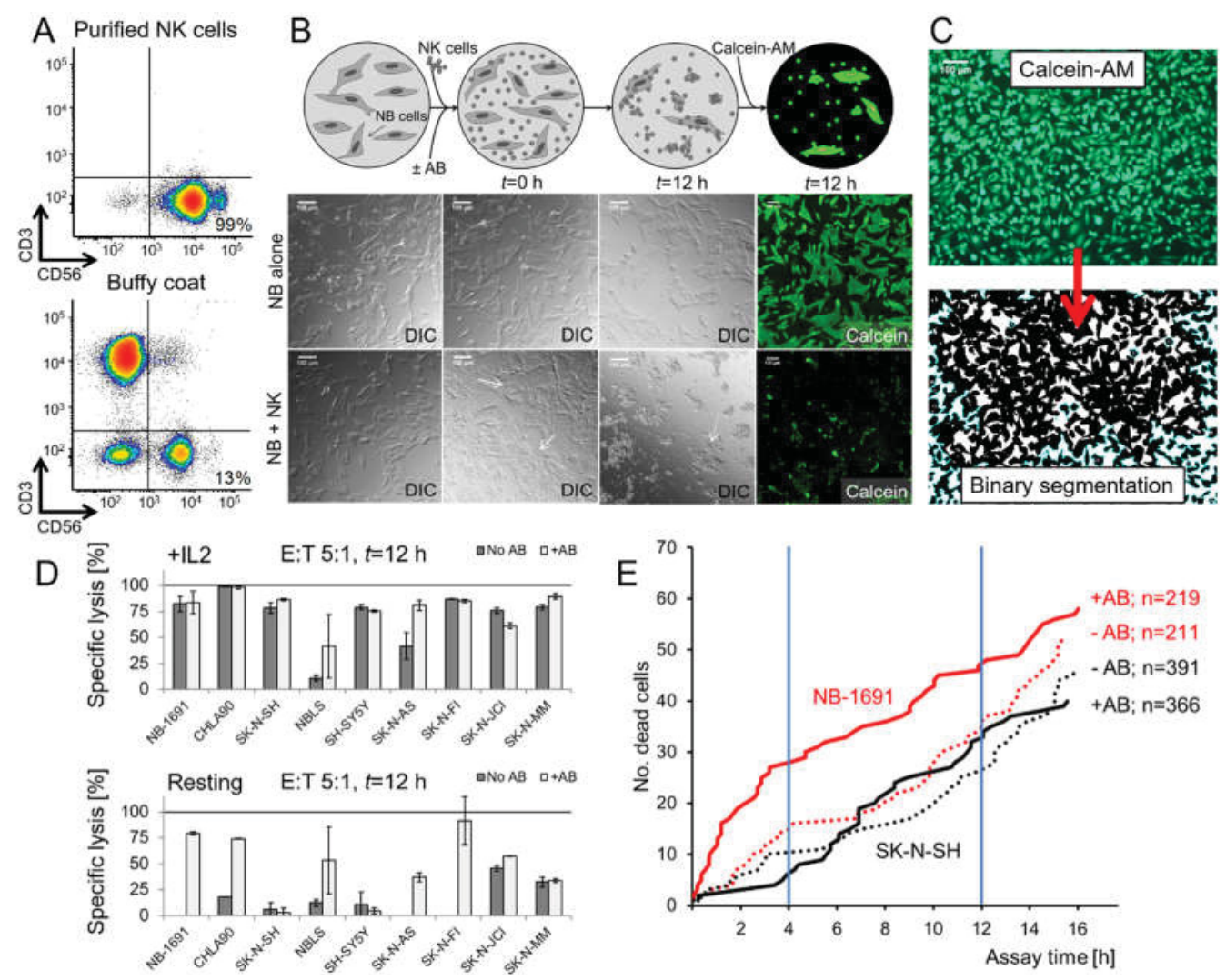

Figure 3-1. Development and validation of an assay to quantify NK cell-mediated cytotoxicity and ADCC in neuroblastoma cells

Notes: (A) Isolation of NK cells from peripheral whole blood yielded a highly purified CD56+/CD3 - population (98\%) compared with that of the buffy coat (13\%). (B) Images produced in DIC microscopy are shown. For the ADCC assay, neuroblastoma cells were plated into a 96-well plate and incubated with NK cells (solid arrow) \pm Hu14.18K322A ( $t$ $=0 \mathrm{~h}$ ). The number of neuroblastoma cells per well was counted to calculate the E:T ratio. After 12 hours $(t=12 \mathrm{~h})$, most NK cells were washed off with a small amount aggregated on neuroblastoma cells (dashed arrow). Viable cells were stained with calcein-AM and imaged by semiautomated microscopy. (C) Binary segmentation of the original image was performed with Fiji to determine cell confluence. (D) The effect of NK cell-mediated ablation (dark gray bars) and ADCC with Hu14.18K322A (light gray bars) was compared in resting and IL-2-activated NK cells (50 IU/mL) across nine neuroblastoma cell lines. The E:T ratio was 5:1 with a 12 hour incubation time. (E) Two neuroblastoma cell lines were studied in co-culture by live-cell imaging. The E:T ratio was 5:1. The number of dead cells with ADCC and NK cell-mediated cytotoxicity was determined over time for NB-1691 (red line) and SK-N-SH (black line). Most significant differences between cell lines were seen with a 12-hour incubation time. AB, antibody; DIC, differential interference contrast; E:T, effector-to-tumor. 
neuroblastoma cell lines with low (SK-N-SH) and high GD2 expression (NB-1691) was quantitated. Specific lysis induced by Hu14.18K322A was significantly higher in NB1691 than in SK-N-SH at 12 hours (both $P<0.001$ ). In absence of Hu14.18K322A, this difference was statistically greater at 12 hours $(P<0.001)$ than at four hours $(P=0.042)$. These conditions were used for all subsequent experiments.

Because dissociated cells from PDXs tend to grow in aggregates, confluence analysis by quantitative microscopy was not ideal to assess cell viability. Instead, the CellTiter-Glo luminescent cell viability assay was used to determine viability relative to the confluence of cells growing in two dimensions (Figure 3-2).

\section{IL-2 improves ADCC and NK cell-mediated cytotoxicity}

Previous studies used PBMCs to demonstrate the enhancing effect of IL-2 on ADCC induced by anti-GD2 antibody against neuroblastoma [130]. Although it is known that IL-2 specifically augments NK-cell function [131, 132], the reported ADCC effect in these studies cannot unambiguously be attributed to NK cells. Purified human NK cells were prestimulated with physiologic and supraphysiologic doses of recombinant IL-2 for 24 hours before conducting ADCC assays. Stimulated NK cells expressed higher levels of NKp30 $(P=0.037), \mathrm{NKp} 44(P<0.001)$, and NKG2D $(P<0.001)$, relative to that of resting NK cells. The cell line SK-N-MM has normal expression of HLA and inhibitory KIR ligands of KIR2DL1, KIR2DL2/3, and KIR3DL1, and has high levels of GD2. This cell line should be least susceptible to KIR-HLA mismatch and therefore most suitable to assess specific lysis due to ADCC by resting and IL-2 activated NK cells at different time points. IL-2 increased ADCC in a dose-dependent manner, and the cytotoxic effect of Hu14.18K322A was most apparent when NK cells were preactivated with $50 \mathrm{IU} / \mathrm{mL}$ IL-2. To quantify ADCC with Hu14.18K322A under these conditions, NK cells from three donors were tested with the nine neuroblastoma cell lines by three serial 1000 -fold dilutions of $\mathrm{Hu} 14.18 \mathrm{~K} 322 \mathrm{~A}$. In resting NK cells, the highest antibody concentration of Hu14.18K322A $(10 \mu \mathrm{g} / \mathrm{mL})$ improved ADCC, compared with that of lower concentrations. The specific lysis was highest with IL-2 IL-2-preactivated NK cells and antibody doses of Hu14.18K322A of $>10 \mathrm{ng} / \mathrm{mL}$. As expected, specific lysis in the GD2-deficient cell line SH-SY5Y did not improve with the addition of Hu14.18K322A. All results are shown in Figure 3-3.

\section{ADCC is more effective than "Missing-self" for NK cell degranulation}

The role of HLA-KIR interactions was examined ex vivo by using two neuroblastoma cell lines that were matched by HLA genotype and GD2 expression but had normal (SK-N-JCI) and low/absent levels of HLA expression (SK-N-SH) compared to white blood cells from buffy coat (normal) and K562 (absent). The ADCC assay was conducted with two concentrations of Hu14.18K322A $(10 \mu \mathrm{g} / \mathrm{mL}$ and $10 \mathrm{ng} / \mathrm{mL})$ and NK cells from 15 allogeneic donors that were prestimulated with $50 \mathrm{IU} / \mathrm{mL}$ of IL-2. As 

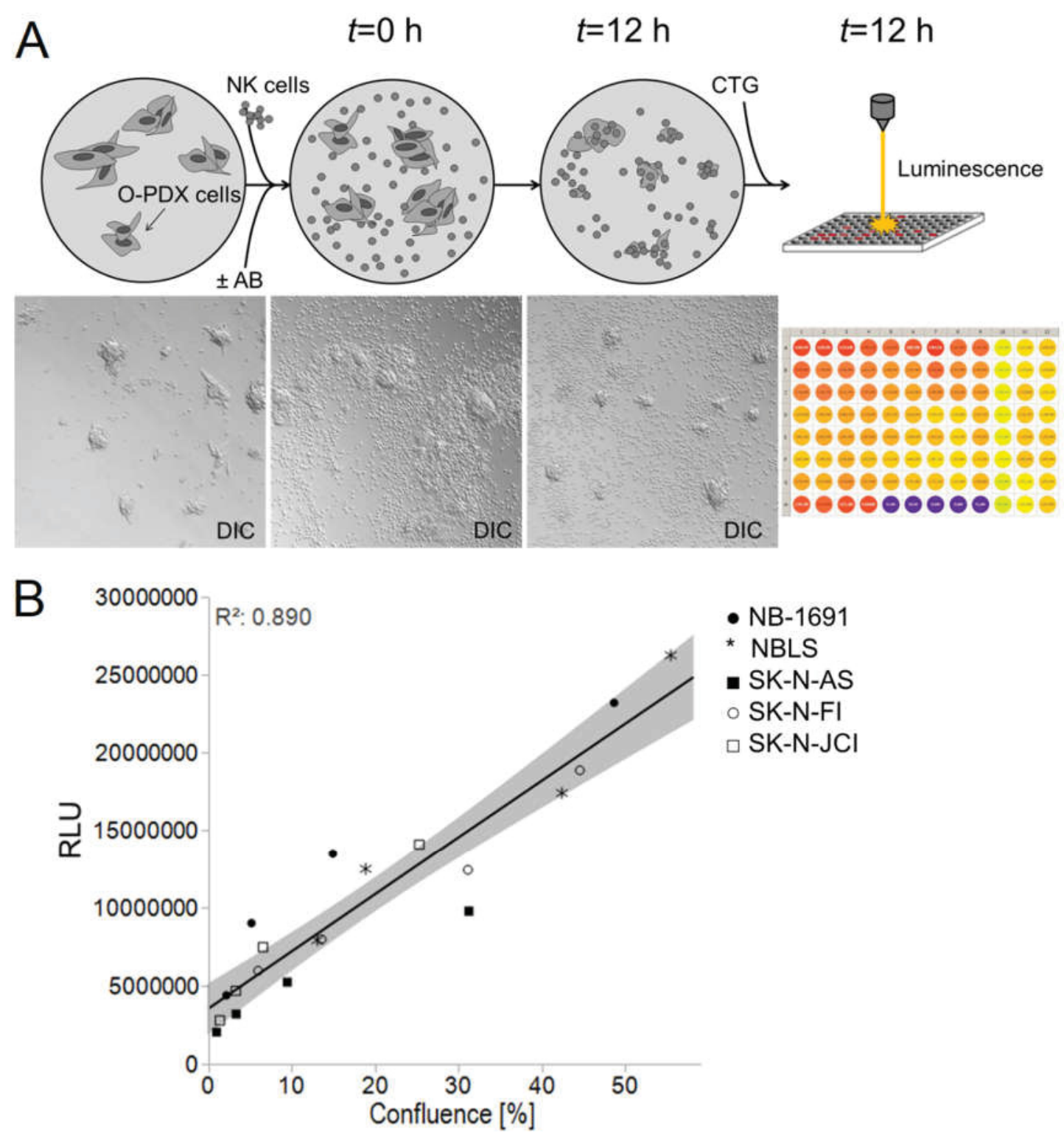

Figure 3-2. Development of an assay to quantify NK cell-mediated cytotoxicity and ADCC in PDXs

Notes: (A) Dissociated tumor cells from PDXs were plated for the ADCC assay. IL-2activated or resting NK cells were added $\pm \operatorname{Hu} 14.18 \mathrm{~K} 322 \mathrm{~A}(t=0 \mathrm{~h})$ and incubated for 12 hours. The CellTiter-Glo luminescent cell viability assay was used to quantify ADCC. (B) ADCC results are plotted as relative light units (RLU) correlate with confluence in cells that are growing in two dimensions. DIC, differential interference contrast; RLU, relative light unit. 

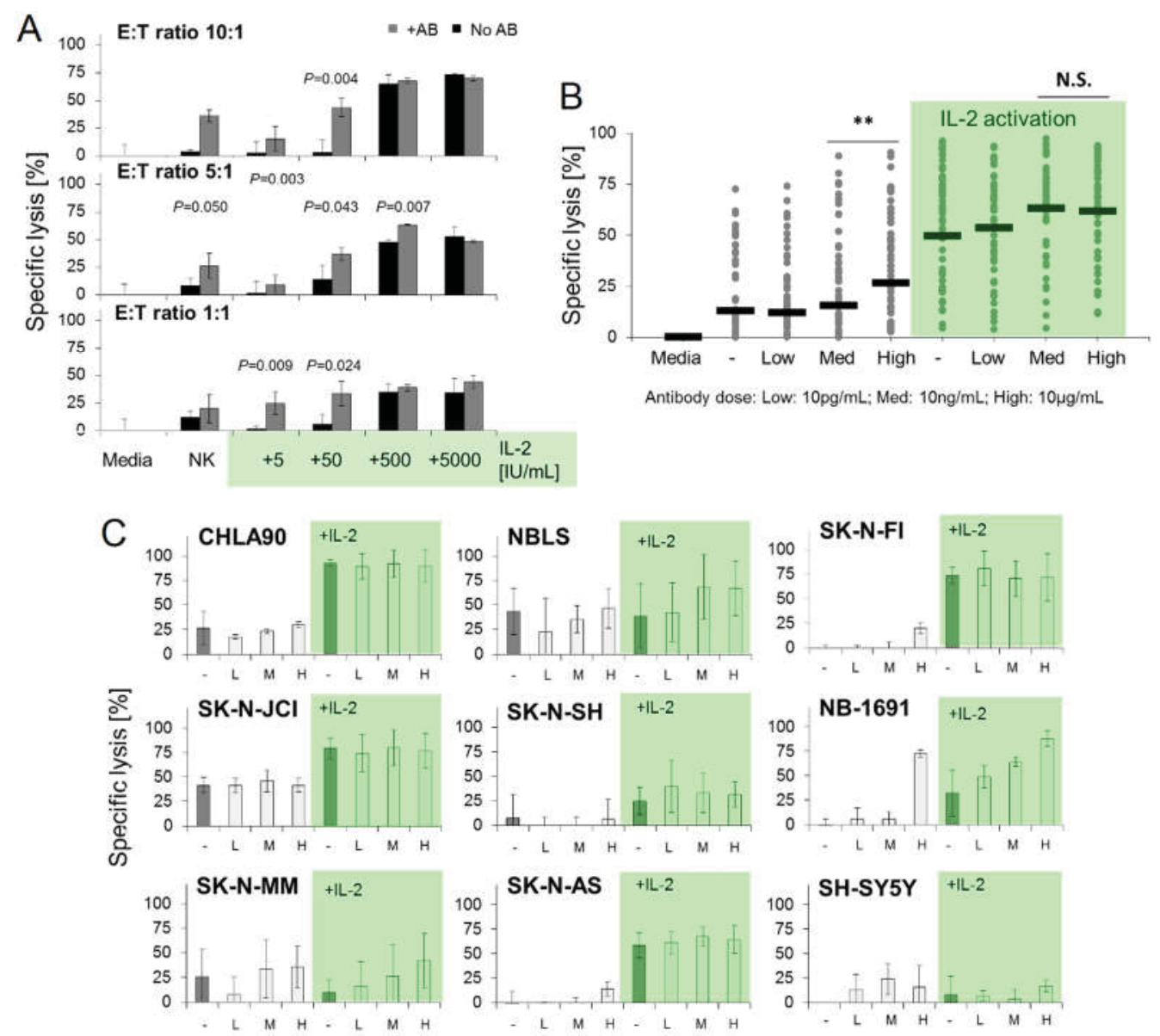

Figure 3-3. IL-2 enhances NK cell-mediated ADCC in neuroblastoma cells

Notes: (A) NK cells were prestimulated with IL-2 concentrations ranging from 0 to 5000 $\mathrm{IU} / \mathrm{mL}$ for 24 hours and tested with the SK-N-MM neuroblastoma cell line at different $\mathrm{E}: \mathrm{T}$ ratios. The assays were conducted with (gray bar) and without Hu14.18K322A (black bar). The cytotoxic effect of Hu14.18K322A was statistically significant across multiple E:T ratios when NK cells were preactivated with $50 \mathrm{IU} / \mathrm{mL}$ IL-2. (B) NK cells from three allogeneic donors were tested in nine neuroblastoma cell lines. Three serial dilutions of Hu14.18K322A were used [High (H): $10 \mu \mathrm{g} / \mathrm{mL}$, Med (M): $10 \mathrm{ng} / \mathrm{mL}$, and Low (L): $10 \mathrm{pg} / \mathrm{mL}$ ] to induce ADCC. The highest antibody concentration yielded better median cytolysis (black bar) in resting NK cells, but a reduced concentration $(10 \mathrm{ng} / \mathrm{mL})$ was equally efficacious when administered with IL-2. (C) Results for individual neuroblastoma cell lines are shown in which IL-2-preactivated NK cells showed equivalent cytotoxicity with lower antibody doses. The highest antibody concentration yielded better median cytolysis in NB-1691 with resting and IL-2-preactivated NK cells. ADCC was comparable to NK cell-mediated cytotoxicity in the GD2-deficient cell line SH-SY5Y. AB, antibody; E:T, effector-to-tumor; IL-2, interleukin-2; NK, natural killer. 
expected, specific lysis by IL-2-activated NK cells was higher in both cell lines, compared with the specific lysis mediated by resting NK cells $(P<0.001$ for both cell lines). When the effect of ADCC was directly compared between the neuroblastoma cell lines, IL-2-stimulated NK cells induced lysis in more of the HLA-expressing neuroblastoma cells (SK-N-JCI) than in the HLA low-expressing cells (SK-N-SH). The mechanisms leading to higher specific lysis in the presence of IL-2 are unclear. It is possible that other receptor-ligand interactions not measured in this study accounted for these differences. To control for these variables, future experiments could use one cell line with conditional HLA knock-out to assess the effect of HLA-KIR interactions on ADCC. The results are presented in Figure 3-4.

Because the ADCC assay only quantitates tumor cell death, CD107a expression was measured in NK cells from three donors to study the impact of HLA-KIR interaction on NK cell degranulation. Using the KIR and HLA genotype information from each donor, the licensed (expressing KIR that corresponds to donor HLA) and unlicensed (expressing KIR which the donor did not express HLA for) NK cell populations were determined. Subsequently, IL-2-activated NK cells were co-incubated with neuroblastoma cells that genotypically lacked the HLA class I molecules corresponding to CD158a (SK-N-AS), CD158b (NBLS), or CD158e1 (NB-1691), and HLA-KIR ligand mismatch was recreated this way. HLA expression was induced by IFN- $\gamma$ treatment for 72 hours. A blocking anti-HLA antibody was used to recreate the state of missing self (lack of HLA-KIR interaction), and Hu14.18K322A was used to induce ADCC. The highest levels of CD107a expression was present in NK cells expressing CD158b $(P$ $<0.001)$, harboring KIRs that were mismatched against HLA expressed by the respective neuroblastoma cells $(P=0.016)$, in the presence of antibody $(P<0.001)$, and in licensed NK cells $(P<0.05)$. KIR expression and KIR-HLA mismatch status remain statistically significant in a multiple regression analysis. Collectively, these data suggest that higher NK cell-mediated activity may be achieved via recruitment of the licensed NK cell population and in the setting of anti-GD2 antibody and KIR-HLA mismatch aside from other signals as shown in Figure 3-4.

\section{Neuronal differentiation enhances ADCC}

Because neuroblastoma tumors comprise neuroblastic cells (N-type), GD2negative Schwann cell-like stroma cells (S-type), or both cell types (I-type), the expression of the neuronal marker GD2 can vary between and within tumors [133]. To determine what effect neuronal differentiation may have on susceptibility to ADCC, neuroblastoma cells were cultured in ATRA for 72 hours before being subjected to the ADCC assay. GD2 expression was determined by flow cytometry, and gene expression of differentiated neuroblastoma cells were compared with that of untreated cells. All cells were assigned a cell type designation (N-, S-, or I-type) according to their morphology.

N-type and I-type cells increased neurite outgrowth, which did not occur in S-type cells. This morphologic change was associated with an up-regulation of genes involved in 
Figure 3-4. Comparison of ADCC and missing-self recognition for neuroblastoma cell ablation

Notes: (A) The SK-N-SH neuroblastoma cell line demonstrated downregulation of HLA (red) surface expression, whereas the SK-N-JCI cell line expressed characteristic HLA levels. Isotype is shown in gray. K562 served as negative control and white blood cells from buffy coat as positive control. (B) Head-to-head comparison of susceptibility to NK cell-mediated cytotoxicity and ADCC. Activated NK cells induced more tumor cell death in SK-N-JCI than in SK-N-SH cells. Resting NK cells did not affect tumor cell death levels. (C) HLA expression was induced by IFN- $\gamma$ treatment for 72 hours and comparable to buffy coat. Degranulation of NK cells from three donors was assessed by quantifying CD107a expression in single KIR-positive NK cell sub-populations after co-incubation with neuroblastoma cells that genotypically lacked the HLA class I molecules corresponding to CD158a (SK-NAS), CD158b (NBLS), or CD158e1 (NB-1691). When stratified by KIR sub-population (D), presence of HLA-KIR mismatch between NK cells and tumor cells (E), use of antibody Hu14.18K322A (F), or licensing status and HLAKIR mismatch $(G)$, the greatest level of NK cell degranulation was noted in CD158bexpressing NK cells (D), in NK cells harboring KIRs that were mismatched against HLA expressed by the respective neuroblastoma cells (E), in the presence of antibody (F), and in licensed NK cells $(\mathrm{G})$. KIR expression and HLA-KIR mismatch status remain statistically significant in a multiple regression analysis. AB, antibody; HLA, human leukocyte antigen; IL-2, interleukin-2; IFN- $\gamma$, interferon gamma; KIR, killer-cell immunoglobulin-like receptor. 


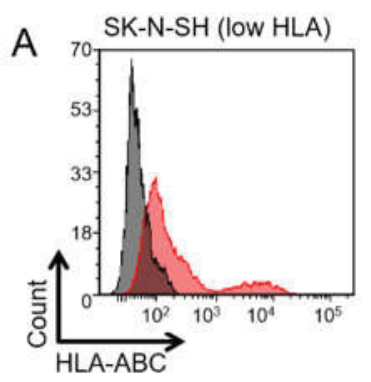

B

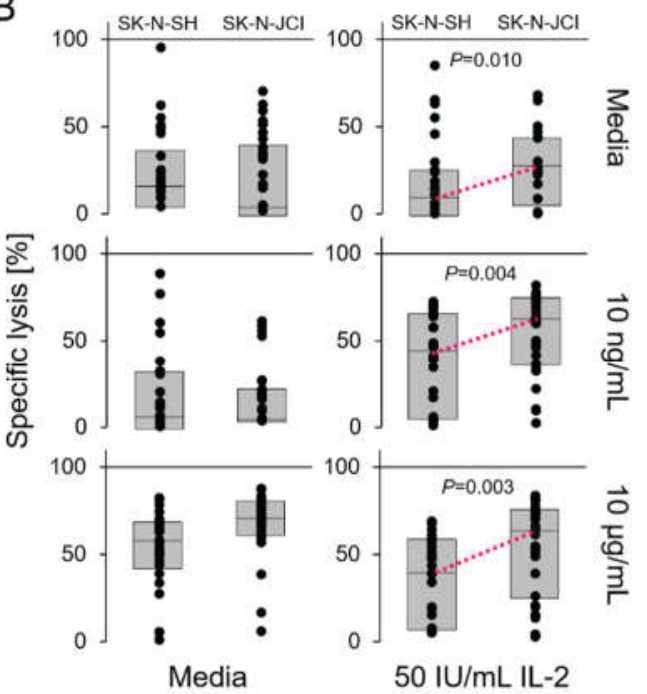

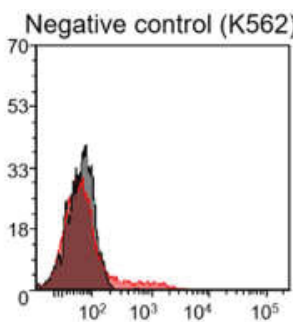

C
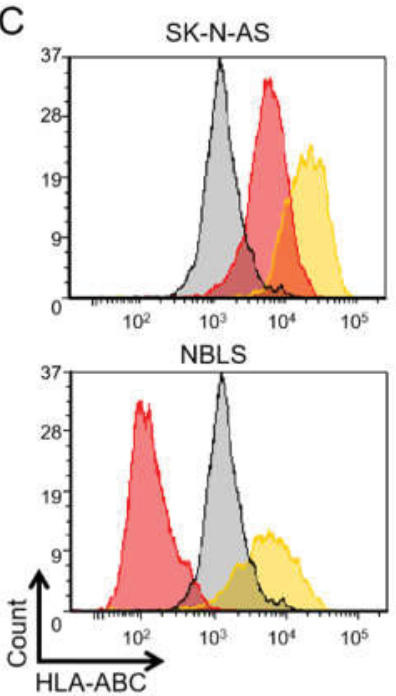

Positive control (Buffy coat)
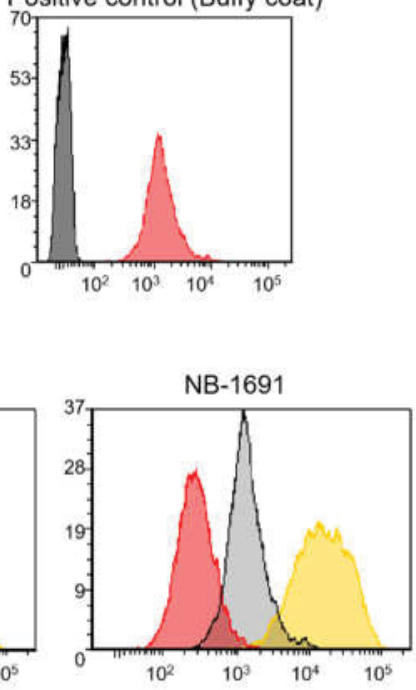

Media, Buffy coat, INFY
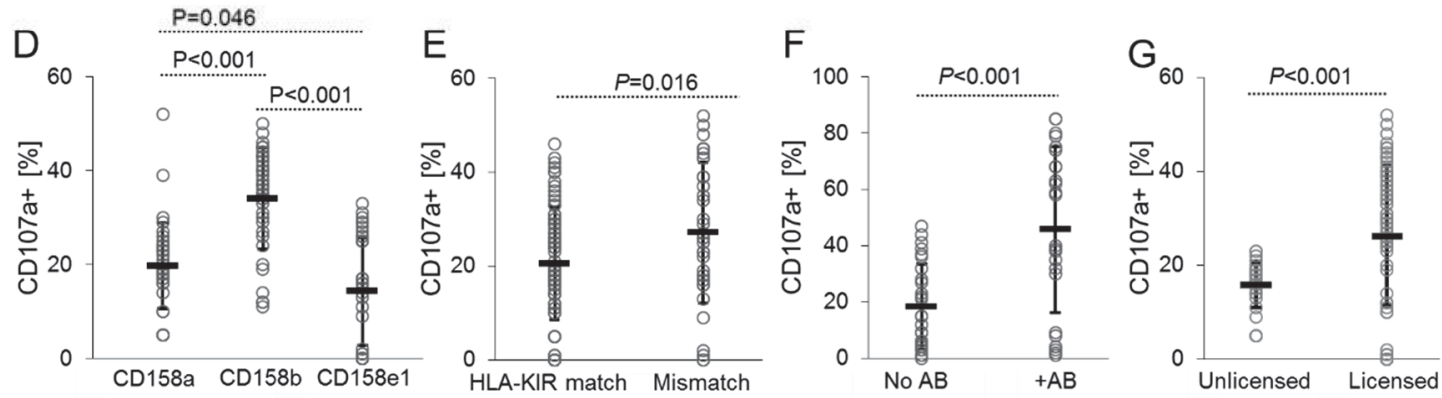
ATRA metabolism (CYP26A1/B1, DHRS3; Fig. 5b) and binding (CRABP1/2, RARB, RBP1; Fig. 5c). Other genes that were found to be differentially expressed in ATRAtreated cells encode for proteins involved in cell structure, the MAPK and NOTCH1 signaling pathway, neuronal differentiation, and angiogenesis.

A high percentage of GD2-positive cells were present among the neuroblastoma cell lines (median, 83.9\%; range, 44.0\%-98.9\%) with exception of SH-SY5Y cells (mean, 2.9\% \pm 0.7 ; Fig. 5d). The percentage of GD2-positive cells did not change after ATRA treatment (median, 78.5\%; range, 1.3\%-98.5\%). The mean fluorescence intensity for GD2 ranged broadly (median, 1001; range, 189-32,461) but did not significantly change after neuronal differentiation (median, 1332; range, 203-16,402; $P>0.05$ ). ADCC was higher in differentiated neuroblastoma cells when induced by resting NK cells $(P=0.004)$. For all other conditions, specific lysis of ATRA-treated cells was not different than that in untreated cells. The results from these experiments are shown in Figure 3-5.

\section{Individualized testing of ADCC is possible with PDXs and matched NK cells from individual patients with neuroblastoma}

To test ADCC of individual patients in the laboratory, ADCC assays were conducted with PDXs and NK cells matched from the same donor. The PDX SJNBL108_X expressed high levels of GD2, as detected by flow cytometry, and a second PDX SJNBL013761_X1 was deficient in GD2 as shown in Figure 3-6. CD158bexpressing NK cells were the predominant KIR-expressing NK-cell population in the peripheral blood of the SJNBL108_X donor, and CD158a-expressing NK cells were predominant for the SJNBL013761_X1 donor. Both PDXs were susceptible to ADCC when induced with Hu14.18K322A, which was unexpected for SJNBL013761_X1 because it did not express any appreciable levels of GD2. This could be due to expression of other compensatory surface molecules, such as HLA class I, although this was not tested. The SJNBL013761_X1 PDX was resistant to NK cell-mediated cytotoxicity without antibody, even when the NK cells were stimulated with IL-2. Live-cell imaging supported this observation (data not shown).

\section{IL-15 enhances natural cytotoxicity and ADCC in neuroblastoma}

Previous studies and these investigations [134] have highlighted the supporting role of IL-2 in increasing NK cell survival and cytotoxicity in neuroblastoma. However, the therapeutic administration of IL-2 has been limited in the clinic because of considerable toxicities, especially at high doses [135]. Previous studies have established the positive effects of IL-15 on NK cell function and development [136-138]. Therefore, IL-15 may be as effective as or more effective than IL-2 in enhancing ADCC against neuroblastoma and has the added benefit of reduced toxicity. This is because GM-CSF which is administered in the existing immunotherapy regimen for neuroblastoma can 
A
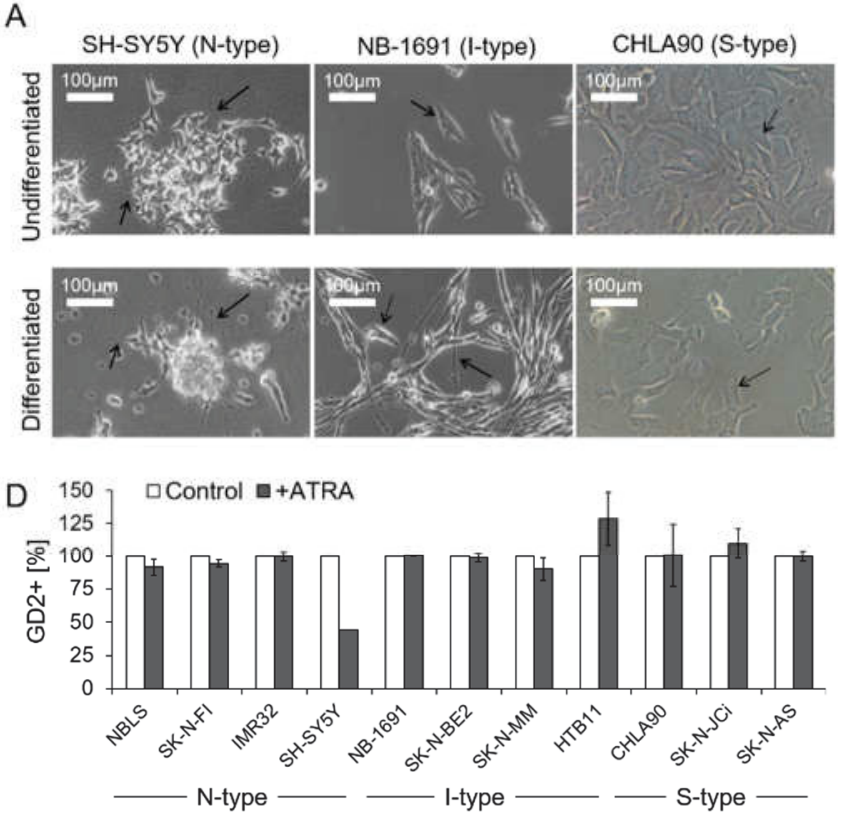

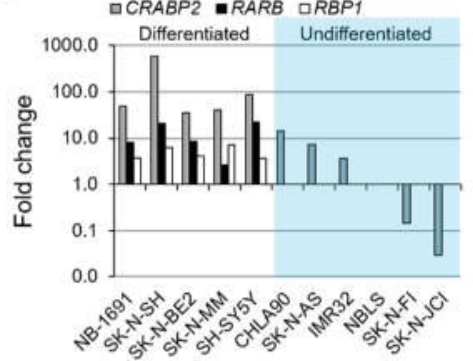

Controls

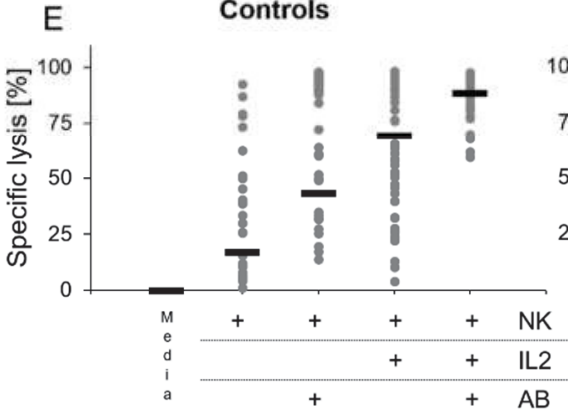

C ICYP26A1 —CYP26B IDHRS3

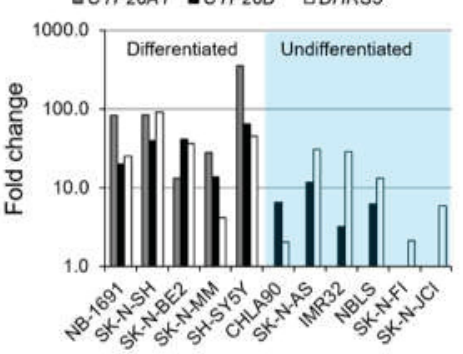

ATRA-treated

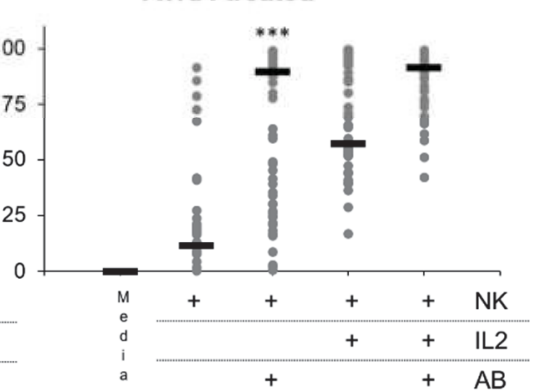

\section{Figure 3-5. Effect of neuroblastoma differentiation on NK cell-mediated ADCC}

Notes: (A) After exposure to ATRA for 72 hours, neurite outgrowth was present in N-type and I-type cells (solid arrows). No changes in morphology were noted in some I-type cells and most S-type cells (dashed arrows). (B) Differential gene expression of ATRAbinding proteins (B) and enzymes involved in ATRA metabolism (C) after differentiation of neuroblastoma cells. (D) For all but two cell lines (SK-NSH and SH-SY5Y), the percentage of GD2-expressing cells after ATRA-induced differentiation was unchanged, compared with that in untreated cells. (E) ADCC assays using two allogeneic donors against 10 neuroblastoma cell lines were conducted. Each dot refers to the average specific lysis per cell line for the respective experimental condition. Significantly $(* * *)$ higher median specific lysis (black bar) by resting NK cells and Hu14.18K322A was noted after differentiation with ATRA. AB, antibody; ATRA, all-trans retinoic acid; IL-2, interleukin-2. 

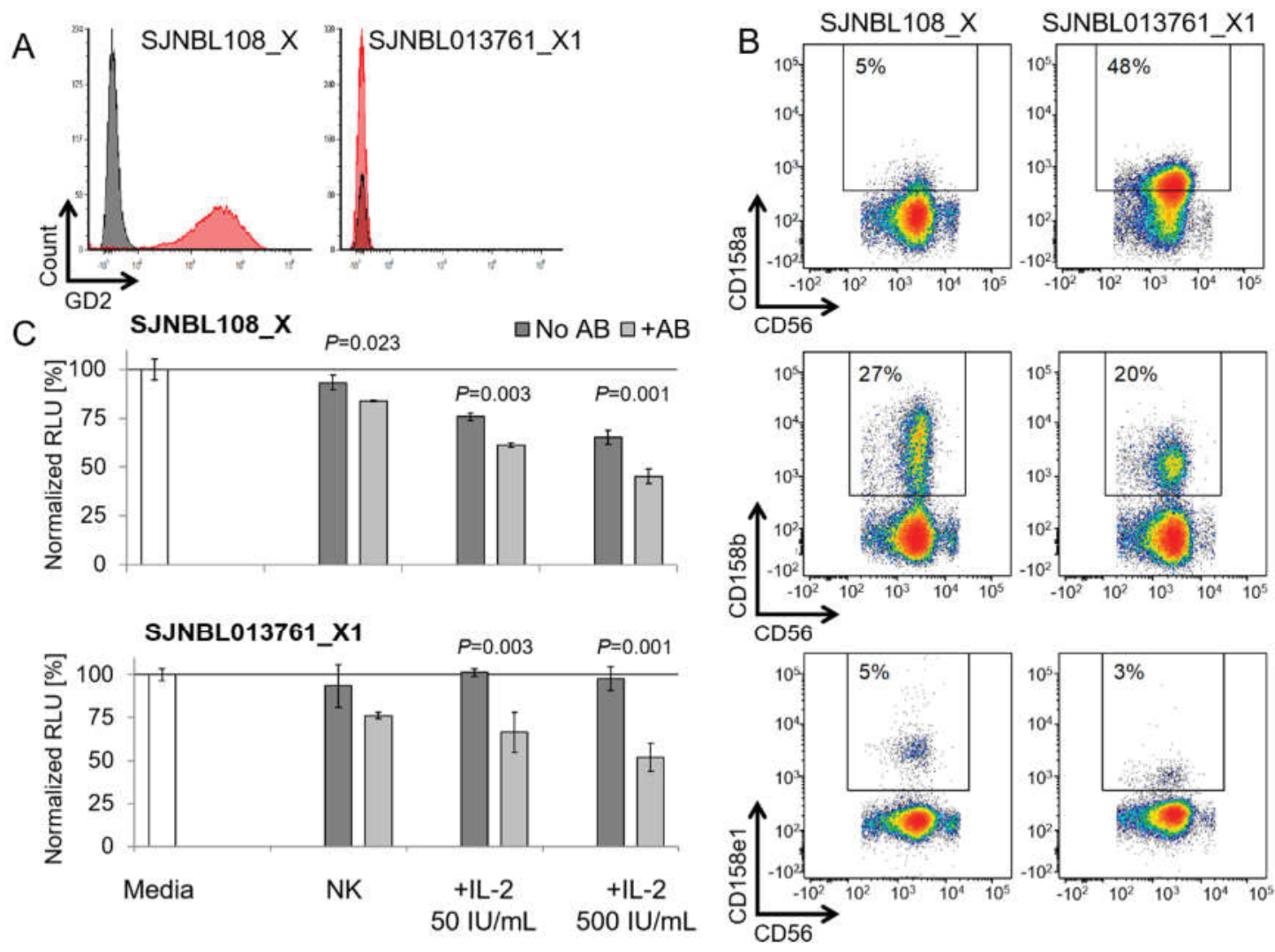

Figure 3-6. ADCC assays with matched O-PDXs and patient NK cells

Notes: Flow cytometry plots are shown depicting GD2 expression (A) and KIR phenotype (B) in the SJNBL108_X and SJNBL013761_X1 PDXs. (C) ADCC assay results for SJNBL108_X and SJNBL013761_X1 are plotted as RLU. AB, antibody; GD2, disialoganglioside; $\mathrm{NK}$, natural killer; RLU, relative light unit. 
induce the mobilization of IL-15 to the plasma membrane of monocytes (transpresentation) [139], thereby increasing its bioavailability.

To compare the differential effect of IL-2 with IL-15 on NK cell-mediated lysis of neuroblastoma cells in culture, NK cells were preactivated with decreasing concentrations of IL-2 ranging from 1 to $50 \mathrm{IU} / \mathrm{mL}$ and IL-15 ranging from 1 to 10 $\mathrm{ng} / \mathrm{mL}$ for 24 hours. These concentrations were chosen because they correspond approximately to steady-state levels in humans treated with less than or equivalent to the maximum tolerated dose of IL-2 [140] and IL-15 [141]. Preactivated NK cells were tested with the PDX SJNBL046_X at multiple E:T ratios and generally yielded higher tumor cell death than resting NK cells at similar E:T ratios. Preactivation with IL-15 increased natural cytotoxicity in a dose-dependent manner that was superior to IL-2 at the highest concentration across all E:T ratios. This effect was maintained with the next lower dose level at an E:T ratio of 5 to 1 . In the presence of Hu14.18K322A, ADCC was equivalent between IL-15 and IL-2-activated NK cells. Results are shown in Figure 3-7.

To confirm these findings, testing was extended to include four donors and two PDX lines, SJNBL046_X and ATRX-mutant SJNBL047443_X. NK cells were stimulated with intermediate doses of both cytokines (i.e., $10 \mathrm{IU} / \mathrm{mL}$ of IL-2 and $5 \mathrm{ng} / \mathrm{mL}$ of IL-15), and an E:T ratio of 5 to 1 was used in the assay. Resting NK cells showed the least cytotoxic activity, whereas NK cell-mediated tumor ablation appeared highest with Hu14.18K322A (10 $\mu \mathrm{g} / \mathrm{mL})$ and IL-15. IL-15 is a chemoattractant for NK cells and augments the binding of NK cells to endothelial cells and fibroblast [142]. Whether IL-15 alters the contact between neuroblastoma and NK cells is unknown. However, to examine the possibility that IL-15 increases NK cell mobility and tumor cell contact, thereby increasing tumor lysis, live-cell imaging was applied to visualize the interaction of NK cells with tumor. Two donors were tested with the neuroblastoma cell lines, CHLA-90 and SK-N-AS. The number of contacts between tumor and effector cells as well as cell death were determined over time and found to be highly correlated with each other (Spearman $\mathrm{r}=0.6 ; P=0.005)$. Although time $(P<0.001)$, donor $(P<0.001)$, and tumor cell line $(P<0.001)$ were significantly associated with tumor and effector cell contact, the type of cytokine stimulation was not $(P=0.248)$. Collectively, these results suggest that the enhanced cytotoxicity induced by IL-15 compared to IL-2-preactivated NK cells against neuroblastoma is not due to differences in cell-to-cell contact but other biologic factors that warrant further investigations.

\section{IL-15 increases the number of tumor-infiltrating NK cells in neuroblastoma spheres}

Neuroblastoma is associated with repressed immunity. This is consistent with the low tumor mutational burden and number of tumor-infiltrating $\mathrm{T}$ lymphocytes (TILs) reported in the literature [143-145]. The lack of immune infiltration in the tumor can be a major obstacle when using immunotherapeutic approaches but may be overcome with tumor-specific interventions, like monoclonal antibodies or CAR-T cells targeting the tumor directly. Alternatively, agents that promote generalized immune activation and improved chemotaxis, such as cytokines, may be useful. To test whether IL-15 and IL-2 


\section{Figure 3-7. Effects of IL-2 and IL-15 on natural cytotoxicity against neuroblastoma and tumor-NK cell contact}

Notes: (A) NK cells were prestimulated with several concentrations of IL-2 and IL-15 for 24 hours and subsequently tested with the PDX SJNBL046_X at different E:T ratios. The assays were conducted with and without Hu14.18K322A. (B) NK cells from four donors were tested in two PDX lines using an E:T ratio of 5 to 1 and intermediate concentrations of IL-2 (10 IU/mL) and IL-15 (5 ng/mL). In all comparisons by donor, IL-15preactivated NK cells with antibody induced the highest cytolysis than resting and IL-2activated NK cells with or without antibody. No difference to IL-15 alone was found in three comparisons and marked accordingly (NS). (C) In this tumor-effector cell contact assay, the contact between neuroblastoma cells and NK cells was determined and

correlated with tumor lysis. The assay was conducted with two allogeneic donors and two neuroblastoma cell lines (CHLA-90 and SK-N-AS). IL, interleukin; NK, natural killer; $\mathrm{NB}$, neuroblastoma; NS, not significant. 

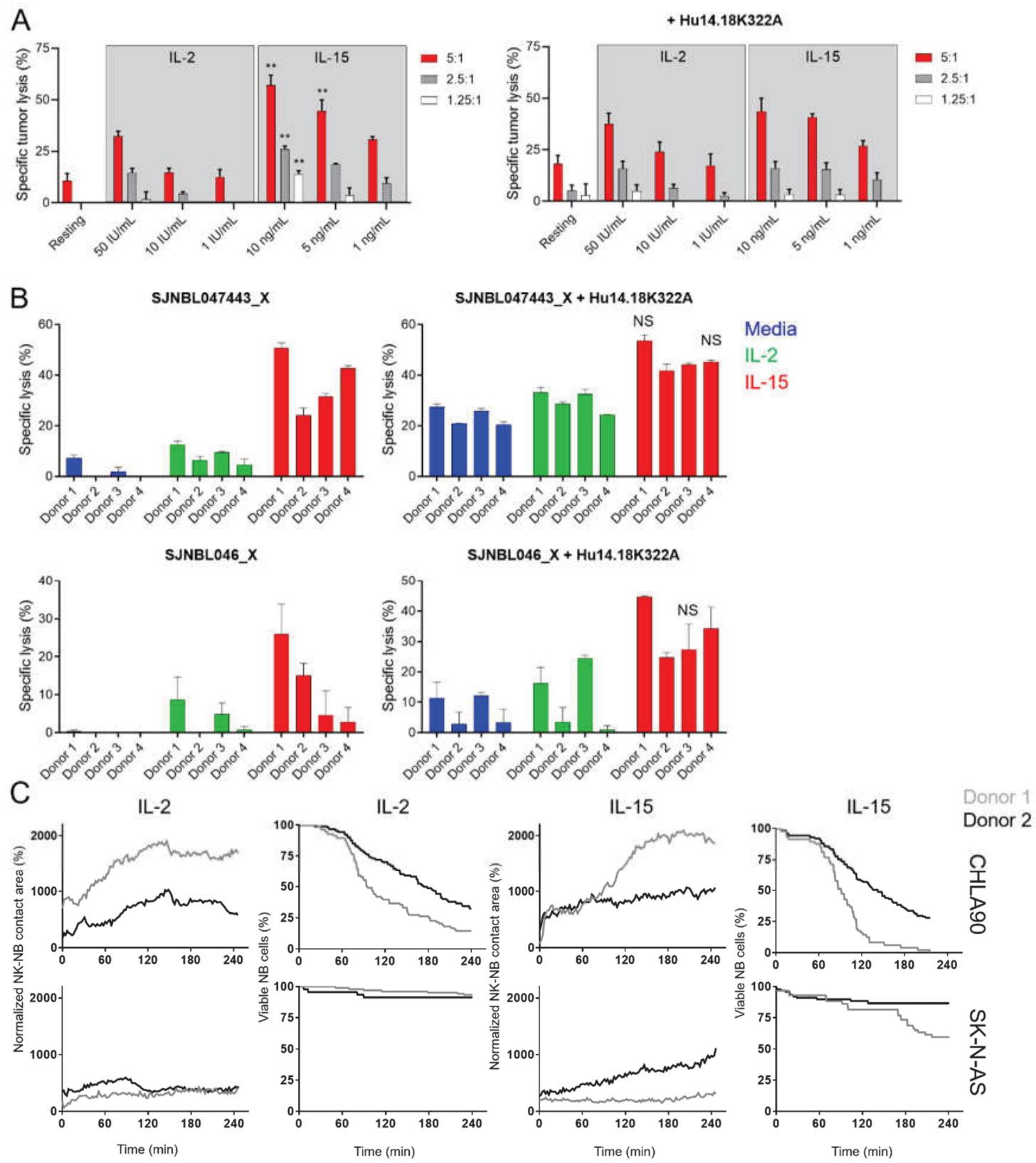
can enhance tumor invasion by NK cells in neuroblastoma, a PDX sphere model was applied. The following experiments were conducted with the immune "cold" PDX line, SJNBL046_X, that, despite visible splenic infiltration, lacks the presence of TILs and tumor-infiltrating NK cells when subjected to multiple different allogeneic effector cells in vivo. Purified NK cells and an estimated E:T ratio of 5:1 were used in the assay. When preactivated with cytokines, sphere-infiltrating NK cells (SPHINKs) were detectable within four hours of culture. Higher numbers of SPHINKs were noted after prestimulation with IL-15 compared to media alone or supplemented with IL-2.

SPHINKs expressed lower levels of NKG2D and TIGIT and higher levels of CD69 than non-infiltrating NK cells. Despite an increased proportion of $\mathrm{CD} 69^{+}$cells, SPHINKs had attenuated cytotoxic activity. Altogether, these findings support that the neuroblastoma microenvironment has an immune-suppressive effect on NK cells, compromising tumor invasion and proper cytotoxic function in culture. Tumor cell-mediated immune suppression can be partially reversed with IL-15 which increases NK cell invasion into the tumor. These results are summarized in Figure 3-8.

\section{The IL-15/IL-15R $\alpha$-complex suppresses growth of neuroblastoma PDX in vivo}

IL-2 and IL-15 mediate through the same IL-2/IL-15 $\beta \gamma$ receptor and have overlapping biologic effects [146]. But unlike IL-2 that can exist as a soluble and unbound molecule in the blood, IL-15 requires IL-15R $\alpha$ to form a complex for transpresentation on the cell surface of activated monocytes [147]. This interaction supports the development and homeostasis of $\mathrm{CD}^{+} \mathrm{T}$ cells and NK cells through STAT5 signaling $[148,149]$. Stimulation with GM-CSF can induce mobilization of IL-15 to the plasma membrane of monocytes [150], and this becomes clinically relevant because GMCSF is currently used with anti-GD2 antibody and IL-2 to treat high-risk neuroblastoma [151]. Although some pre-clinical studies have demonstrated enhanced ADCC in the presence of IL-15 [152-154], the supportive role of IL-15 in neuroblastoma therapy is unclear. To test if IL-15 can substitute IL-2 in immunotherapy for neuroblastoma, an in vivo efficacy experiment was conducted that evaluated the anti-tumor effect in four experimental groups with PDX-bearing CD1-Foxn $1^{\text {nu }}$ mice. Mice either received chemotherapy alone (group 1), or chemotherapy followed by 14G2a antibody and GMCSF (group 2), and IL-2 (group 3) or IL-15/IL-15R $\alpha$-complex (group 4). Although CD1Foxn $1^{\text {nu }}$ mice are immunocompromised, they have decreased but functional levels of endogenous monocytes/macrophages and NK cells.

Animals who received chemoimmunotherapy with IL-15/IL-15R $\alpha$ had the most suppressed tumor response among all 4 therapy groups (Figure 3-9). In direct comparison, mice in group 4 had smaller tumors than those treated with chemotherapy alone $(P=0.01)$, chemotherapy followed by $14 \mathrm{G} 2 \mathrm{a}$ antibody and GM-CSF $(P=0.02)$, and chemotherapy followed by $14 \mathrm{G} 2 \mathrm{a}$ antibody, GM-CSF, and IL-2 $(P=0.007)$. Analysis of spleen and tumor tissue by flow cytometry revealed that the percentage of immature murine NK cells was marginally lower in the group 4 mice compared to all other therapy groups $(P=0.064)$ and untreated animals $(P=0.039)$. No differences were found in the profile of tumor or spleen-infiltrating monocytes and macrophages. 

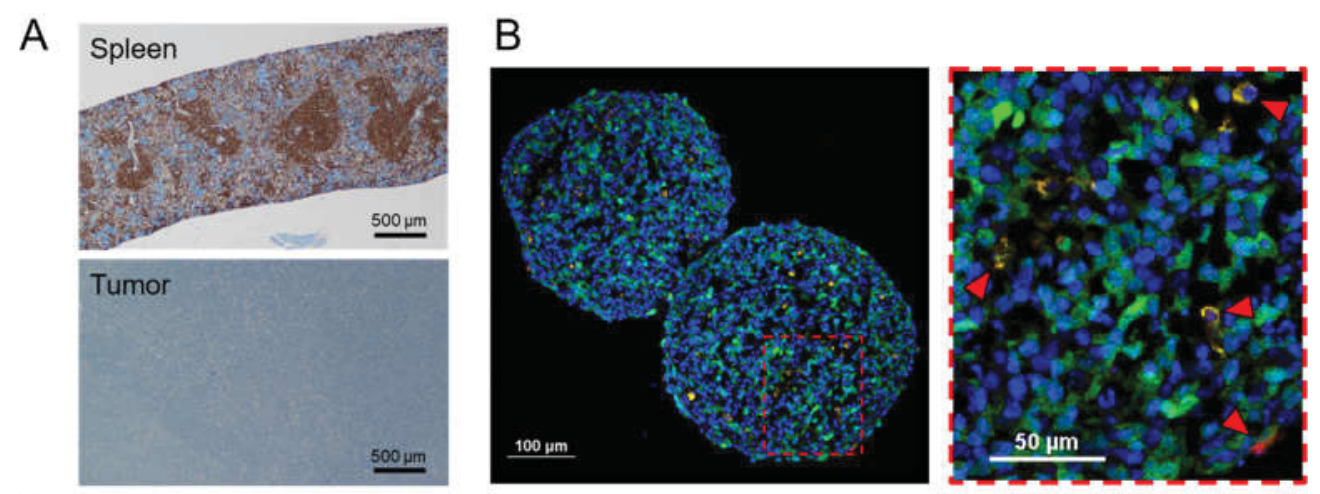

C
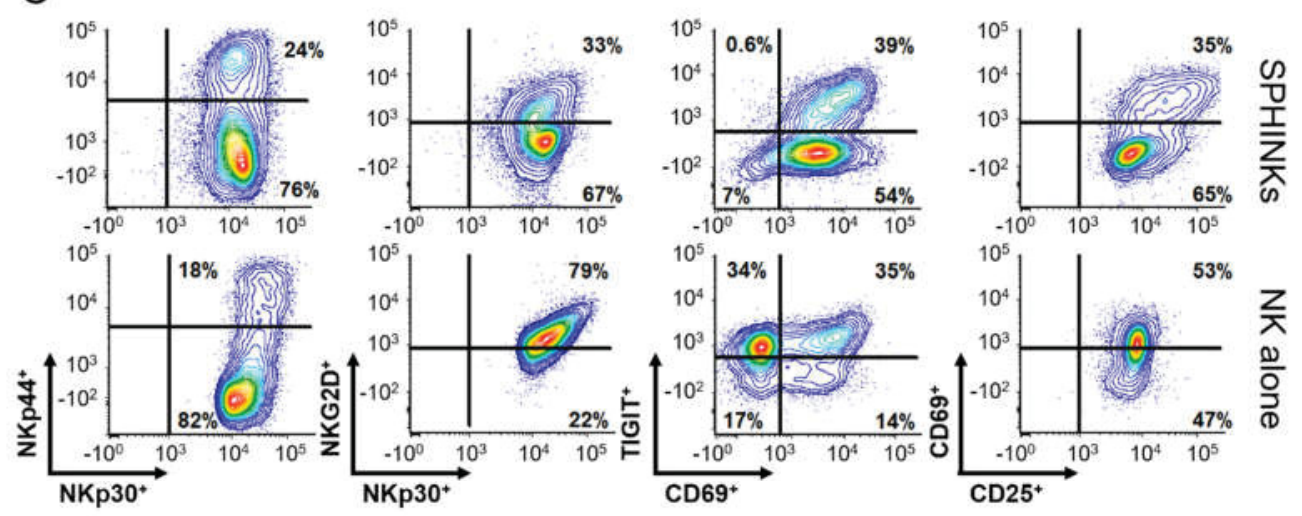

\section{$\frac{2}{\lambda}$}

D

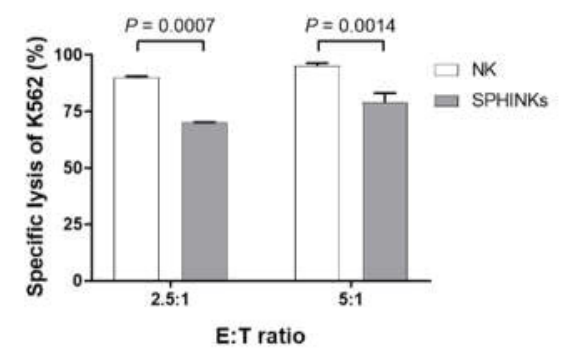

$E$

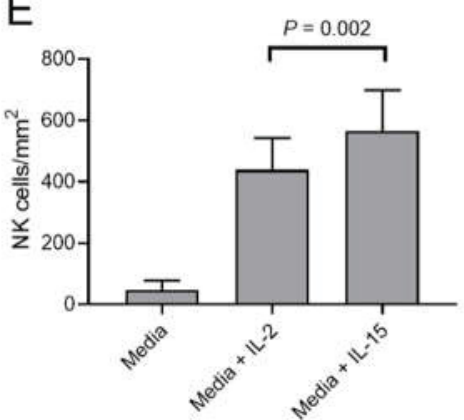

Figure 3-8. Phenotype and function of tumor-sphere-infiltrating NK cells (SPHINKs)

Notes: (A) Yellow fluorescent protein-expressing PDX cells (green) were used to form tumor spheres. NK cells (orange) are capable of infiltrating tumor spheres (red arrows). DAPI stain was performed for nuclear discrimination. (B) Phenotypic characterization of SPHINKs show a decreased expression of NKG2D and TIGIT and higher proportions of CD69-expressing cells compared to non-infiltrating NK cells. (C) Isolated SPHINKs have decreased cytotoxicity against K562 compared to non-infiltrating NK cells. (D) The number of SPHINKs is higher when NK cells are activated with IL-15 than IL-2 or media alone. E:T, effector-to-tumor; NK, natural killer; SPHINKs, tumor-sphere-infiltrating NK cells. 
Figure 3-9. Comparison of IL-15/IL-15Ra-complex with IL-2 as immunoadjuvant agent in immunotherapy for neuroblastoma

Notes: (A) Tumor growth over time in all 4 therapy groups. (B) Gating strategy to identify CD $11 b^{+}$Ly6G $^{-} \mathrm{Ly}_{6 \mathrm{C}^{+}} \mathrm{MHC}^{-} \mathrm{II}^{-}$and $\mathrm{CD} 11 \mathrm{~b}^{+} \mathrm{Ly}_{6 \mathrm{G}}^{-} \mathrm{Ly}^{-} \mathrm{C}^{-} \mathrm{MHC} \mathrm{II}^{+}$monocytes and macrophages. (C) Percentage of $\mathrm{CD}^{11 \mathrm{~b}^{+} \text {Ly6G- }}{ }^{-}$cells of all $\mathrm{CD}^{-} 5^{+}$cells in spleen (blue) and tumor (red). Percentages of CD11 b ${ }^{+} \mathrm{Ly}_{6-} \mathrm{Ly}^{-} \mathrm{C}^{+} \mathrm{MHC} \mathrm{II}^{-}$and $\mathrm{CD}^{11 b^{+}}$ $\mathrm{Ly}_{6 \mathrm{G}^{-} \mathrm{Ly}^{-} \mathrm{C}^{-} \mathrm{MHC} \mathrm{II}^{+} \text {in spleen (D) and tumor (E). (F) Gating strategy to identify Ly6C }}$ $\mathrm{DX}^{+} \mathrm{CD} 27^{+} \mathrm{CD} 11 \mathrm{~b}^{-}$immature NK cells and Ly6C $\mathrm{C}^{-} \mathrm{DX} 5^{+} \mathrm{CD} 27^{-} \mathrm{CD} 11 \mathrm{~b}^{+}$mature NK cells. (G) Percentage Ly6C $\mathrm{C}^{-} \mathrm{DX} 5^{+} \mathrm{NK}$ cells of all $\mathrm{CD}^{-} 5^{+}$cells in spleen (blue) and tumor (red). Percentages of immature and mature NK cells in spleen $(\mathrm{H})$ and tumor (I). 

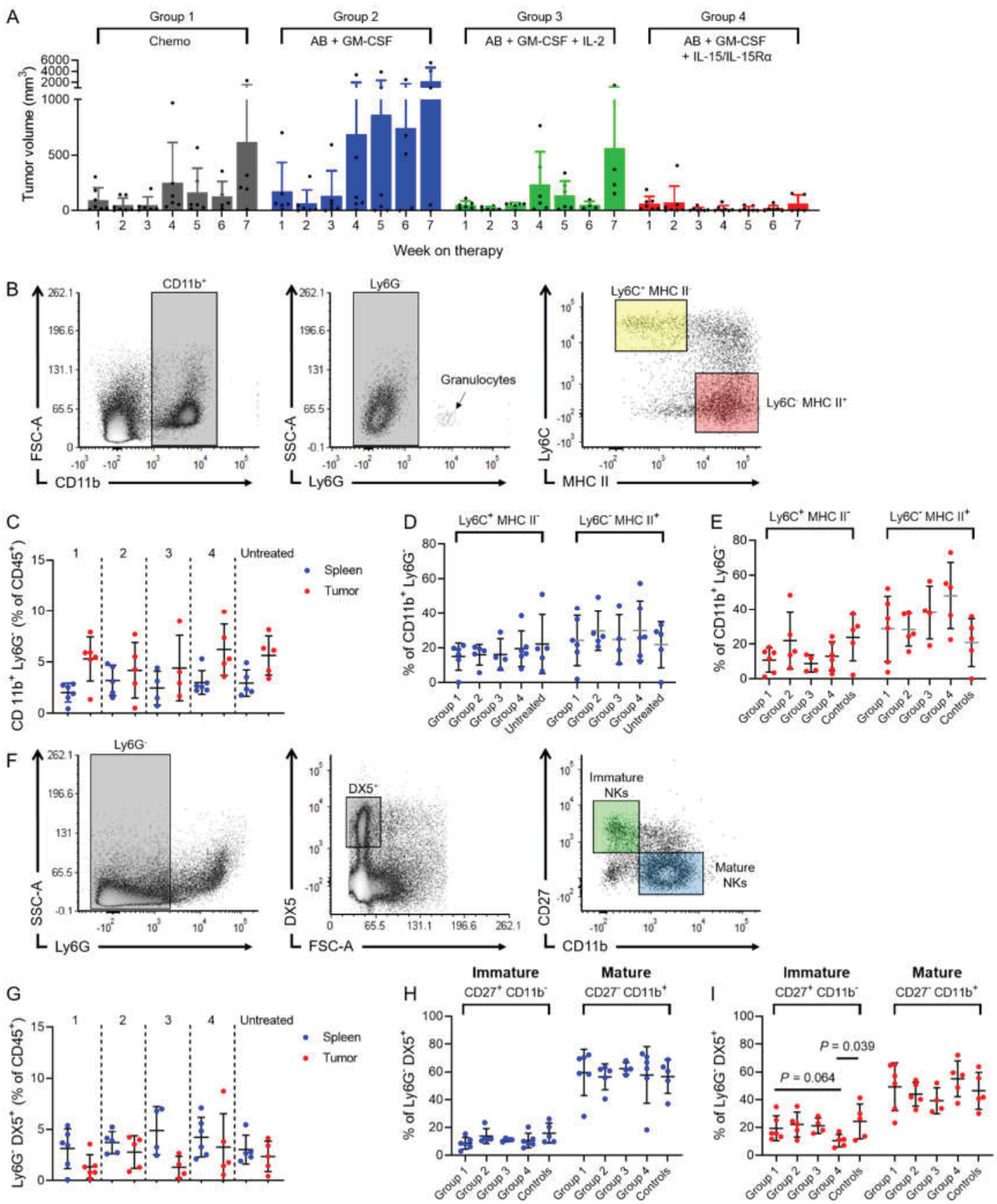


\section{Objective 2}

\section{Engraftment and function of MISTRG-derived NK cells}

In the original report of MISTRG mice, transplantation procedures were conducted in newborn pups [115]. Accordingly, to study the engraftment and function of MISTRG-derived NK cells, newborn MISTRG mice received sublethal doses of radiation within 48 hours after birth and hepatic injections of human peripheral blood-derived $\mathrm{CD} 34^{+}$hematopoietic progenitor cells from G-CSF-mobilized human donors [111] (Figure 3-10). Littermates lacking the SIRPA transgene (i.e., MITRG and NSG mice) served as control strains. I performed the experiment with four allogeneic donors. I detected human hematopoiesis in the peripheral blood at 6 weeks and increased hematopoiesis at 9 weeks after transplantation. At 9 weeks, blood engraftment of human CD $45^{+}$cells was higher in MISTRG mice than in MITRG $(P=0.001)$ and NSG mice $(P$ $=0.003)$. The percentage of human $\mathrm{CD} 6^{+} \mathrm{CD} 3^{-} \mathrm{NK}$ cells, as a fraction of all CD $45^{+}$ cells, was also higher in MISTRG mice than in MITRG $(P=0.003)$ and NSG $(P=0.042)$ mice at that time. The NK cells that developed in these animals expressed mature NK cell surface markers, such as CD16 and NKp46.

To test the cytotoxic activity of human NK cells from neuroblastoma-bearing MISTRG mice, FACS-isolated NK cells from spleens were prestimulated with IL-2 (50 $\mathrm{IU} / \mathrm{mL}$ ) and tested with K562 cells ex vivo. Animals were euthanized 4 to 8 weeks post transplantation when they appeared pale and tumor status was noted. Specific lysis of K562 cells ranged from 2\% to 91\%. Marked variability in cytotoxicity was seen among donors, as well as within littermates reconstituted with $\mathrm{CD} 34^{+}$cells from the same donor. Cytotoxicity was similar in animals with and without tumor $(P>0.05)$. Therefore, MISTRG mice provide a human cytokine environment that is permissive for efficient reconstitution with human $\mathrm{CD} 45^{+}$cells after bone marrow transplantation. Although this observation validates the findings of the original report [115], I also provide evidence that cytokine levels may be insufficient to render intact cytotoxic function in NK cells in the presence of neuroblastoma.

\section{Double-transplantation protocol in adult MISTRG mice}

Although MISTRG mice tolerated hematopoietic progenitor cell transplantation as pups and tumor injections as adults, their longevity was limited by the development of severe anemia at approximately 10 weeks, as previously reported [115]. In my experience, some animals succumbed to anemia before appreciable tumor growth, and of those that were tumor-bearing, the anemia was exacerbated by experimental manipulations, such as by administration of cytotoxic chemotherapy. To test extend the life span of mice in this model, I developed a modified transplantation protocol, in which I performed consecutive hematopoietic transplantation and tumor injections in 4-week adult mice (Figure 3-11). This schedule yielded engraftment with a human immune system and human tumors by approximately 8 to 10 weeks. To compare early 

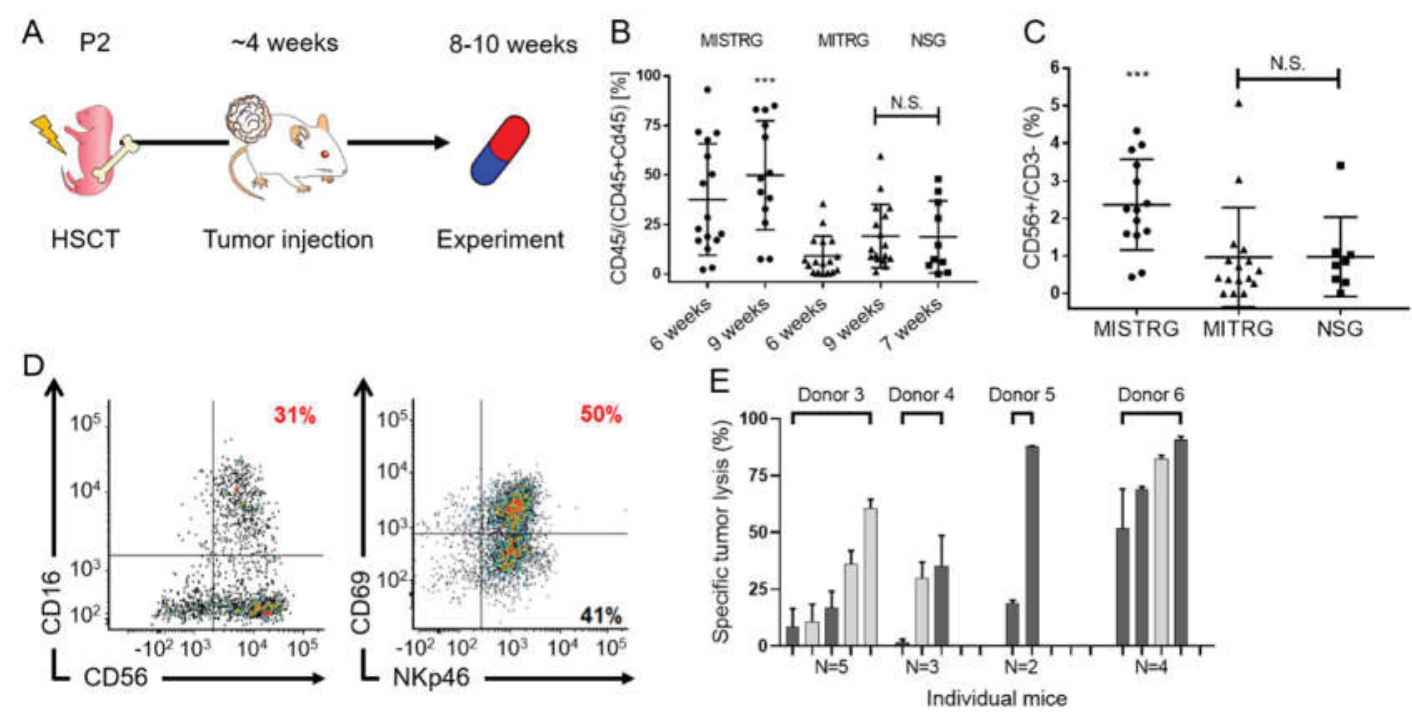

Figure 3-10. NK cell engraftment and function in MISTRG mice after perinatal transplantation

Notes: (A) For the perinatal transplantation protocol, newborn pups were sublethally irradiated within 48 hours after birth and subjected to hepatic injection with CD34+ hematopoietic progenitor cells. At 4 weeks, PDX cells were orthotopically injected into the para-adrenal space. At 8 to 10 weeks, double-transplanted animals developed human hematopoiesis, and the tumors can be used for short-term experimental procedures. (B) Human engraftment levels in the peripheral blood are shown for MISTRG, MITRG, and NSG mice after perinatal transplantation with four donors. At 9 weeks, blood engraftment of human CD45+ cells was higher in MISTRG mice than in MITRG $(\mathrm{P}=0.001)$ and NSG mice at 7 weeks ( $\mathrm{P}=0.003$; Kruskal-Wallis and Dunn test $).(\mathrm{C})$ The proportions of CD56+ CD3 - NK cells of all human CD45+ cells are plotted by mouse strain and are higher in MISTRG mice at 9 weeks post transplantation than in MITRG $(P=0.003)$ and NSG $(\mathrm{P}=0.042$; Kruskal-Wallis and Dunn test). (D) Human NK cells derived from MISTRG mice have a mature phenotype and express CD16, NKp46, and the nonspecific activation marker CD69. (E) FACS-isolated NK cells from the spleens of transplanted MISTRG mice were stimulated with IL-2 $(50 \mathrm{IU} / \mathrm{mL})$ for 12 to 18 hours and tested in a natural cytotoxicity assay against K562 cells. Each bar represents one animal. Tumor bearing mice are marked by dark grey and tumor-free animals by light grey bars. Variability of cytotoxic activity is observed between donors and among NK cells from littermates that were reconstituted with the same donor 


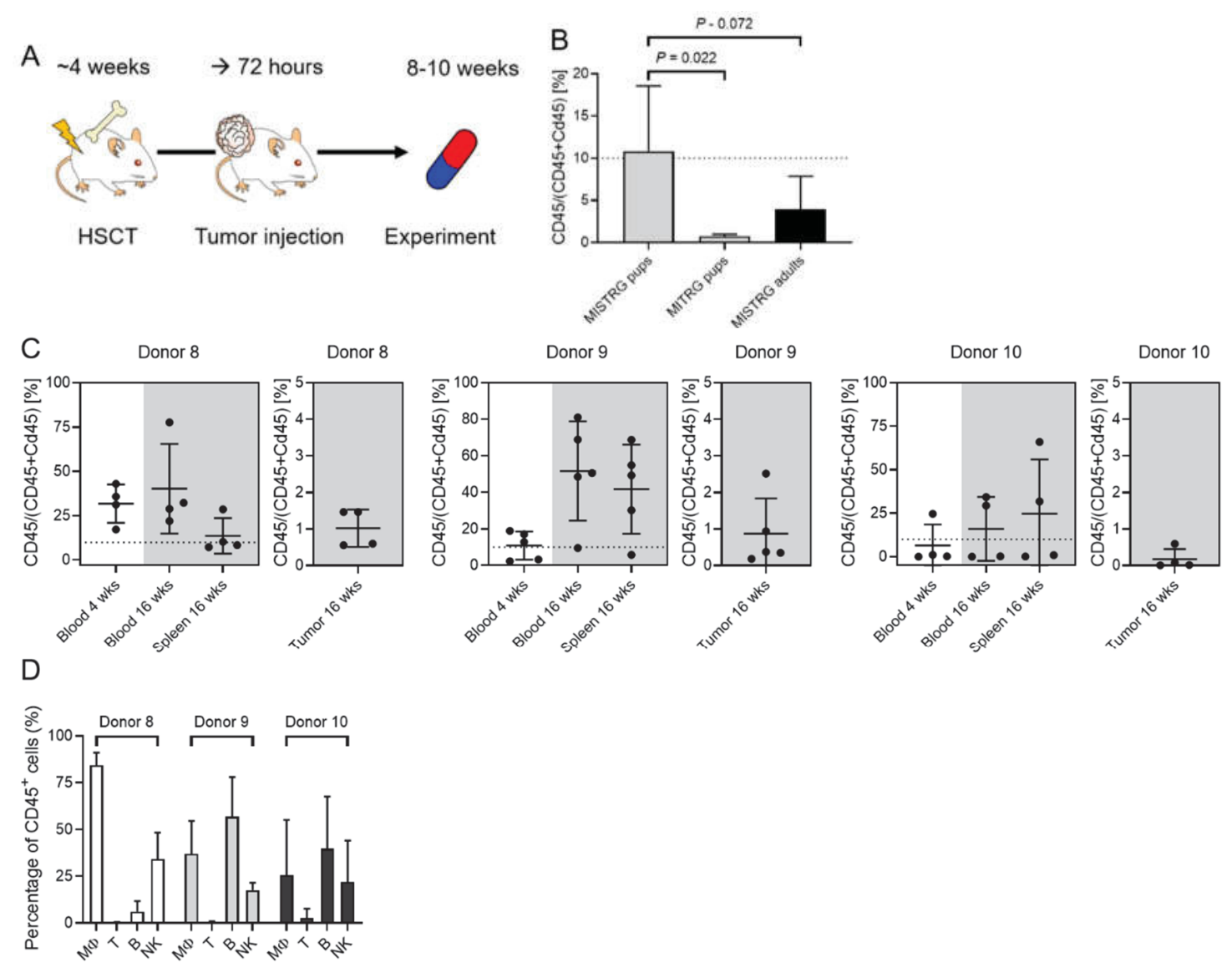

Figure 3-11. Modified transplantation protocol to induce double-engraftment with human hematopoiesis and tumor in adult MISTRG mice

Notes: (A) As per the adult mouse transplantation protocol, animals aged 4 weeks were sublethally irradiated and injected with mobilized peripheral blood CD $34^{+}$hematopoietic progenitor cells intraperitoneally. Para-adrenal PDX cell injection occurred 72 hours after transplantation. (B) Engraftment levels are exemplarily shown for MISTRG and MITRG mice who underwent perinatal (pups) and adult transplantation with $\mathrm{CD} 34^{+}$cells derived from the same donor. Early engraftment levels at four weeks after transplantation were significantly higher in MISTRG compared to MITRG mice. This difference was marginally higher when compared to adult MISTRG animals. (C) Three groups of animals were reconstituted with $\mathrm{CD} 34^{+}$cells from three different donors and reconstituted with the same PDX SJNBL046 X as per the adult transplantation protocol. Early engraftment levels at four weeks in blood and late levels at 16 weeks in blood, spleen, and tumor are shown. These results demonstrate that adult MISTRG mice can sustain long-term engraftment after double-transplantation. (D) Peripheral blood CD45 ${ }^{+}$cells at 16 weeks post transplantation were comprised of monocytes, B cells, and NK cells. MФ, monocytes/macrophages, NK, natural killer; wks, weeks. 
engraftment efficiency at 4 weeks in both transplantation protocols, a cohort of mice received $\mathrm{CD}_{3} 4^{+}$cells from the same donor either as pups (MISTRG and MITRG mice) or as adults (MISTRG mice). The percentage of peripheral blood human CD45 ${ }^{+}$cells reached significantly higher levels after perinatal transplantation in MISTRG mice than in MITRG mice $(P=0.022)$. Marginally higher engraftment levels occurred in perinatal transplantations than in the adult transplantations $(P=0.072)$. However, mice that were transplanted as adults exhibited increased and sustained peripheral blood and organspecific engraftment at 20 weeks. The human $\mathrm{CD} 45^{+}$cells comprised monocytes, NK cells, and B cells but lacked substantial numbers of $\mathrm{T}$ cells because of the absence of a thymus or secondary human lymphoid tissues in these animals that are sites for $\mathrm{T}$ cell development.

Tumor-infiltrating $\mathrm{CD} 45^{+}$cells were barely detectable, but I expected levels of these cells to be low in this PDX line because immune cells from several donors are incapable of infiltrating this PDX tumor in vivo (data not shown). Although reconstituted animals were hemodynamically stable and tolerated therapeutic interventions, a higher mortality rate occurred during procedures requiring anesthesia or blood sampling, which was most likely due to subclinical anemia. Taken together, the humanized MISTRG neuroblastoma model with modified transplantation time line permits long-term human hematopoiesis and concomitant human tumor engraftment.

\section{Molecular and functional analyses of NK cells derived from MISTRG mice with neuroblastoma}

To validate the MISTRG neuroblastoma model, I performed detailed molecular and functional analyses of human NK cells from MISTRG mice that received transplantation as adults and as pups. The four mice received $\mathrm{CD} 34^{+}$cells from two donors (MISTRG 1 and MISTRG 2A, B, C) as adults (MISTRG 1, 2A, and 2C) and pup (MISTRG 2B). MISTRG 2B and 2C received hematopoietic progenitor cell injections from the same cell aliquot. Neuroblastoma tumor-bearing mice with hematopoietic progenitor cell transplantation and tumor injections as adults were euthanized when their tumors measured $10 \mathrm{~mm}^{3}$ to $200 \mathrm{~mm}^{3}$ and splenomegaly was evident via ultrasound, indicative of human hematopoiesis. I removed the spleens and nonenzymatically processed them through a $40-\mu \mathrm{m}$ cell strainer to yield a single-cell suspension (Figure 3-12). $\mathrm{CD} 45^{+} \mathrm{CD} 56^{+} \mathrm{CD} 3^{-} \mathrm{NK}$ cells were isolated via FACS. Targeting 10,000 NK cells per sample, I analyzed a total of 17,801 spleen NK cells from MISTRG mice.

I aligned the single-cell RNA seq data from all mice and found that population density and distribution overlapped between MISTRG 2B and 2C, whereas MISTRG 1 and 2A were separated into distinct clusters (Figure 3-13). Integrated analysis of the aligned data identified the CD56 $6^{\mathrm{dim}} \mathrm{NK} 1$ and CD56 ${ }^{\text {bright }} \mathrm{NK} 2$ cluster, of which the latter one accounted for $50 \%$ to $80 \%$ of the NK cell population in the individual mice. The proportions of NK1 and NK2 cluster corresponded with the expression of GZMB to define the NK1 subset and GZMK for the NK2 subset. When compared to the NK2 population, the NK1 cluster was enriched for transcripts that encoded proteins involved 


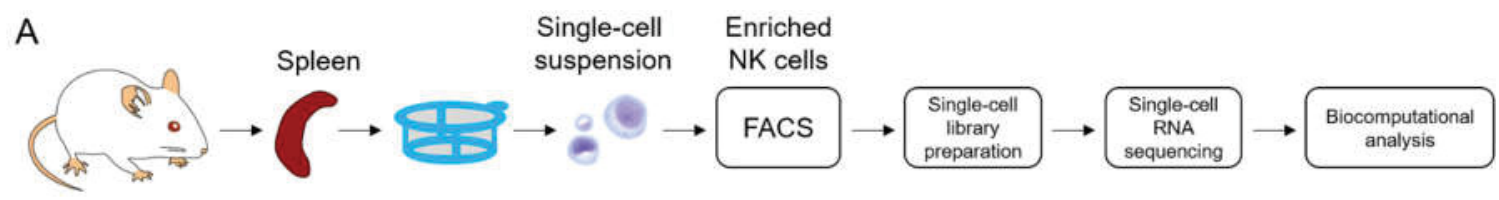

B

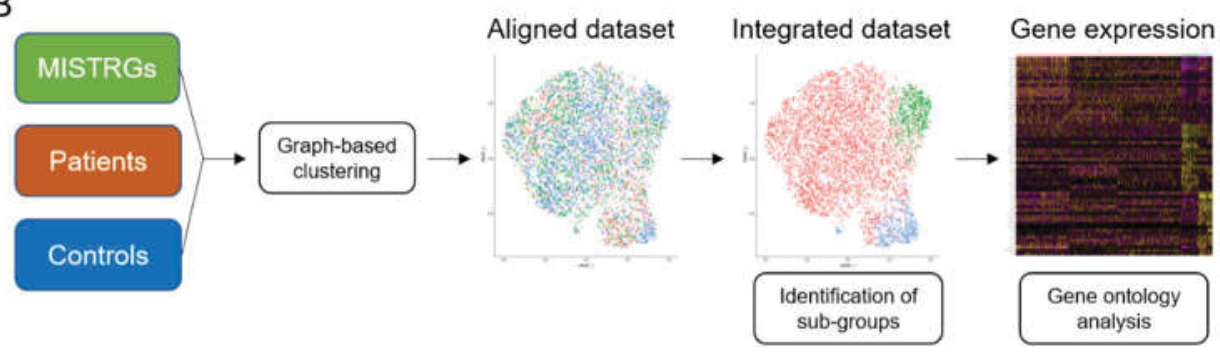

Figure 3-12. Work flow of single-cell RNA sequencing

Notes: (A) Overview of single-cell isolation and NK cell enrichment for single-cell RNA sequencing. (B) Analysis work flow of single-cell TNA sequencing data. FACS, fluorescence-activated cell sorting; NK, natural killer. 
Figure 3-13. Single-cell transcriptome analysis of NK cells from humanized MISTRG mice with neuroblastoma

Notes: (A) Aligned single-cell RNA-seq data sets that combine splenic NK cells from four MISTRG mice using t-distributed stochastic neighbor embedding. The four mice received $\mathrm{CD}_{3} 4^{+}$cells from two donors (MISTRG 1 and MISTRG 2A, B, C) as adults (MISTRG 1, 2A, and 2C) or pup (MISTRG 2B). (B) Visualization of CD56 ${ }^{\mathrm{dim}} \mathrm{NK} 1$ and CD56 ${ }^{\text {bright }}$ NK2 cluster of combined splenic NK cells from four MISTRG mice. (C) Relative percentages of CD56 $6^{\mathrm{dim}} \mathrm{NK} 1$ and CD $56^{\text {bright }} \mathrm{NK} 2$ cells per mouse. The absolute numbers of cells are plotted for each cluster. (D) Color-scaled expression of GZMB and GZMK. (E) Top genes that are differentially expressed between the NK1 and NK2 subset and listed by function. (F) Lysis of K562 cells ex vivo by mouse. (G) Aligned single-cell RNA-seq data sets that combine splenic NK cells from MISTRG 2B (pup transplantation) and $2 \mathrm{C}$ (adult transplantation). (H) Top 10 genes that are differentially expressed between the NK1 and NK2 subset and listed by function (striped bar: cytotoxicity; black bar: cytokine production; white bar: NK cell regulation; grey bar: other). Analysis was performed with NK cells from MISTRG mouse transplanted as pup and adult. 

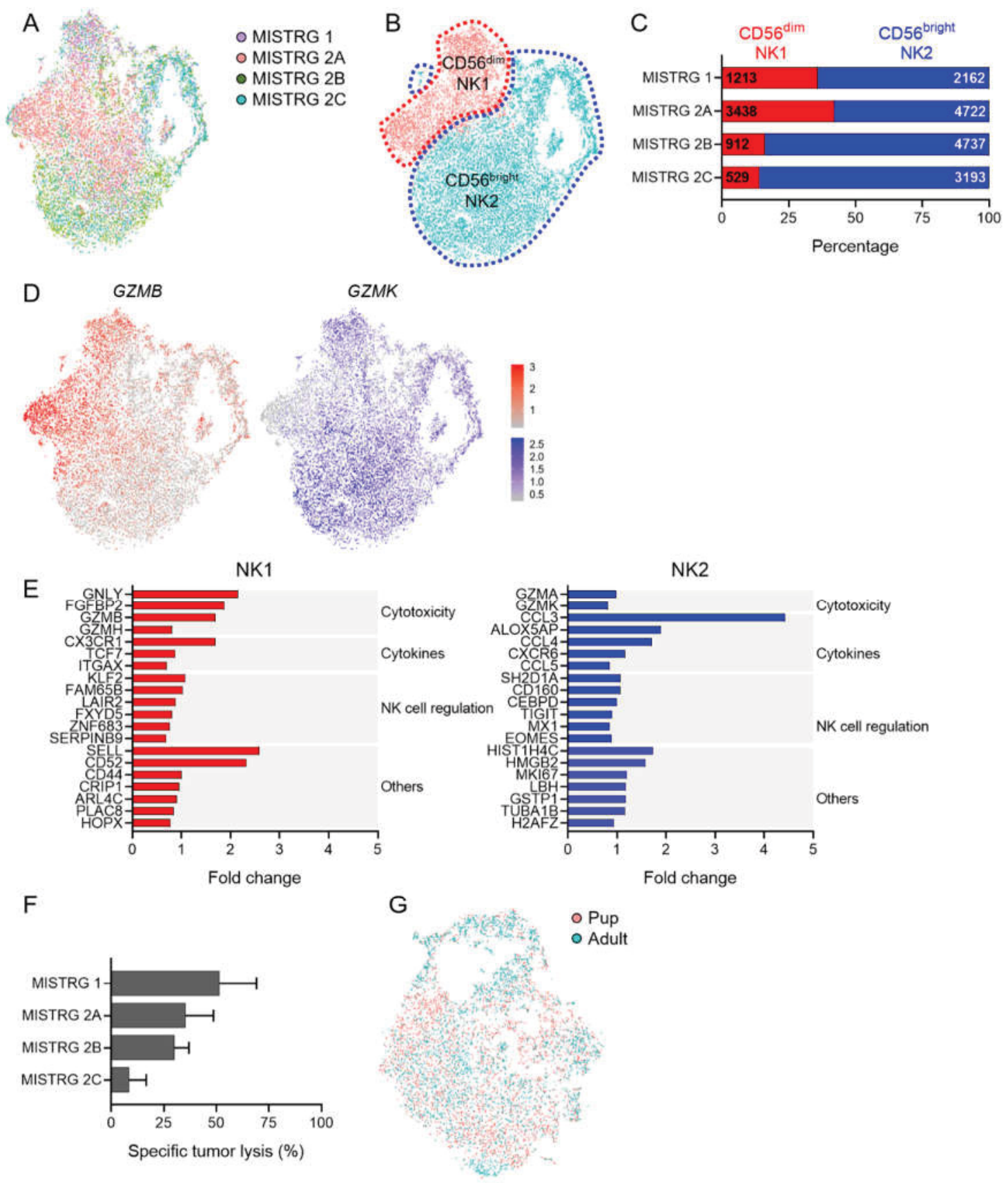

H Pup transplantation

Adult transplantation
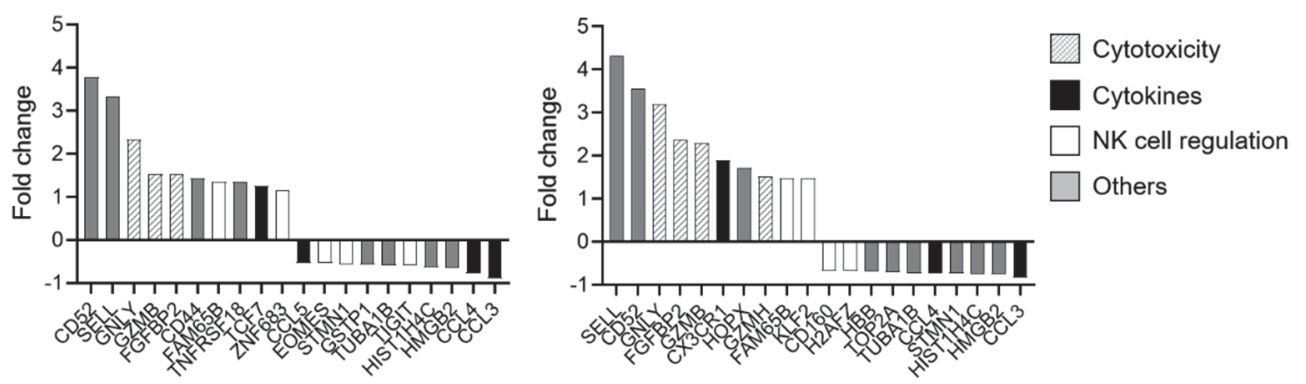
in cytotoxicity (e.g., $G N L Y, F G F B P 2, G Z M B$, and $G Z M H$ ), as well as proteins that are involved in cytokine-mediated processes (e.g., $C X 3 C R 1$, and TCF7) and NK cell regulation (e.g., KLF2, LAIR2, and FAM65B). The most upregulated gene was SELL that is important for recruitment of NK cells to tumors in vivo [155]. Enriched transcripts in the NK2 cluster were associated with cytotoxicity (e.g., GZMA, GZMK, and KLRB1), and NK cell regulation (e.g., $C E B P D, C D 160, S H 2 D 1 A$, and TIGIT). The most upregulated genes encode for cytokines and cytokine receptors or proteins involved in cytokine production (e.g., CCL3, ALOX5AP, CCL4, and CXCRO) that aligns with the biologic function of CD56 $6^{\text {bright }} \mathrm{NK}$ cells. Among the differentially expressed genes in the MISTRG NK1 subset, eight genes were shared by mice and patients (FGFBP2, GZMB, GZMH, CX3CR1, GPR183, TCF7, SELL, and CD44). Among the genes in the MISTRG NK2 cluster, seven genes were shared (GZMK, GZMA, KLRB1, CCL3, CCL4, CLL5, and $A L O X 5 A P)$. Unlike patients whose NK cell function correlated with the proportions of NK1 and NK2 subsets, I observed no molecular-functional correlation in the MISTRG mice.

To determine if transplantation in adult versus newborn mice alters the transcriptome of MISTRG-derived human NK cells, I compared the gene expression profile of NK cells from MISTRG 2B and 2C. These two animals were transplanted as adult and pup using the same $\mathrm{CD} 34^{+}$cell source, and therefore thought to be adequate for such a comparison. When both data sets were combined, I saw that NK cell population density and distribution overlapped between MISTRG 2B and 2C. The proportions of the CD56 ${ }^{\text {dim }} \mathrm{NK} 1$ and CD56 ${ }^{\text {bright }} \mathrm{NK} 2$ cluster were comparable in both animals and accounted for about $20 \%$ and $80 \%$, respectively. I then examined the differential gene expression between the NK1 and NK 2 subpopulation in both data sets. I found that 12 of the 20 most differentially expressed genes involved in cytotoxicity, chemotaxis-related processes, and NK cell regulation were shared between MISTRG 2B and 2C.

Collectively, the transcriptomic analysis revealed that the NK cell gene expression profile in MISTRG neuroblastoma mice is like that in patients with neuroblastoma. Although I have shown that the functional status of patient NK cells correlates with the relative proportion of NK1 and NK2 clusters, the molecular and functional characteristics are unrelated in the humanized MISTRG mice. Therefore, next, I performed a functional assessment of NK cells in vivo.

\section{Comparison of adoptive NK cells with endogenously produced human NK cells in MISTRG mice}

To assess the cytotoxic function of human NK cells in transplanted MISTRG mice in vivo, a cohort of animals first received $\mathrm{CD} 34^{+}$hematopoietic progenitor cells from one donor according to the adult transplantation protocol. Transplanted MISTRG mice then received ultrasound-guided orthotopic injections with the PDX line SJNBL108_X, which is deficient in HLA and expresses high-levels of GD2 (data not shown). Because previous humanized animal models commonly use adoptively transferred human NK cells to target neuroblastoma in NSG, I decided to compare the antitumor immunity of MISTRG-derived NK cells to adoptive human NK cells in 
MISTRG and NSG mice. Therefore, a second cohort of MISTRG mice did not undergo hematopoietic transplantation but received orthotopic tumor injections.

Because tumor engraftment rates are approximately $80 \%$ with this PDX line, I enrolled mice with established tumor volumes measuring $\geq 10 \mathrm{~mm}^{3}$ by ultrasound and randomized the respective animals to receive either chemotherapy or chemoimmunotherapy. Chemotherapy, consisting of daily irinotecan and temozolomide for 5 days, was administered alone or in combination with immunotherapy, which comprised daily hu14.18K322A and IL-2 for 5 days of the following week. With each course of immunotherapy, I injected animals who did not undergo hematopoietic transplantation with $5 \times 10^{6}$ human NK cells from one healthy donor that had intact cytotoxicity prior to cryopreservation and after the thawing process (data not shown).

Because preclinical pharmacokinetic studies have not been conducted with hu14.18K322A in vivo, I performed a flow cytometry-based antibody saturation assay to quantify antibody binding in tumor tissues (Figure 3-14). I found that hu14.18K322A bound to nearly $100 \%$ of the tumor cells by 2 days after antibody injection and gradually decreased over a course of 12 days, which is similar to previously published hu14.18K322A pharmacokinetic findings in humans [92]. The mean fluorescence intensity followed this trend. Thus, I confirmed that hu14.18K322A distributed properly in tumor tissue and excluded poor pharmacokinetics as a possible reason for therapy failure.

All animals injected with human $\mathrm{CD} 34^{+}$or mature NK cells had low but detectable residual levels of human $\mathrm{CD}_{4} 5^{+}$cells in their tumors at the end of therapy. Human $\mathrm{CD} 45^{+}$cell levels were higher in MISTRG mice that received transplantation than those that received adoptive NK cell transfer $(P=0.01)$. I confirmed the presence of $\mathrm{CD}_{56}{ }^{+} \mathrm{CD}^{-} \mathrm{NK}$ cells in these animals.

I performed baseline tumor measurements before therapy start and after two therapy courses lasting 3 weeks each. All mice had macroscopic tumors at the end of therapy, irrespective of the type of intervention or source of effector cells. However, MISTRG mice with endogenous human hematopoiesis treated with chemoimmunotherapy exhibited significantly lower residual tumor burdens than did animals that did not receive bone marrow transplantation but received adoptively transferred NK cells with every course of immunotherapy $(P=0.003)$. Among the animals given chemotherapy alone, no statistically significant difference in tumor burden occurred between the two groups at the end of treatment.

To determine whether the lack of antitumor efficacy with adoptive NK cells was due to strain-intrinsic factors, I repeated the experiment in NSG mice. I treated PDXbearing animals with chemotherapy alone or a combination with immunotherapy and adoptive NK cell injections. I did not observe a statistically significant difference in tumor burden at the end of therapy between the two treatment groups $(P>0.05)$, suggesting that the lack of antitumor effect in adoptively transferred NK cells was not due to strain-specific factors. 
Figure 3-14. Comparison of adoptive NK cells with endogenously produced human NK cells in MISTRG mice

Notes: (A) Antibody distribution and kinetics are examined in tumor-bearing NSG mice that received one dose of $4 \mathrm{mg} / \mathrm{kg}$ of hu14.18K322A. Tumors were analyzed at selected time points by flow cytometry for the presence of antibody. Nearly $100 \%$ of hu14.18K322A-bound tumor cells were detected 2 days after injection. The percentages of antibody-bound tumor cells and mean fluorescence intensities (B) gradually decreased over the following 12 days. (C) Residual tumor-infiltrating human CD45 ${ }^{+}$cells at the end of therapy are shown for mice with endogenous human hematopoiesis and contrasted to mice that received adoptive NK cell injections. Transplanted animals had significantly more infiltrating cells ( $P=0.01$; Mann-Whitney test). (D) Associated flow cytometry plots and gating strategy to quantitate human $\mathrm{CD} 45^{+}$cells and human NK cells from tumor samples. (E) Experimental cohorts were grouped by intervention (chemoimmunotherapy and chemotherapy) and separated by the source of effector cells into transplanted mice (red) and mice that received adoptive transfer of mature human NK cells (blue). Tumor volumes at the start of therapy (on therapy) and after completion of therapy (off therapy) are plotted by group. Among mice receiving chemoimmunotherapy, transplanted MISTRG mice $(N=8)$ had more significant tumor growth suppression than did animals that received adoptive NK cells with therapy $(N=5$; $P=0.003$; Mann-Whitney test). No significant differences occurred between these two groups treated with chemotherapy (each $N=6$ ). (F) Longitudinal tumor volumes in NSG mice treated with chemoimmunotherapy $(N=5)$ and adoptive NK cell injections $(N=5$; left) or chemotherapy alone (right). No statistically significant differences were present in longitudinal tumor volumes between the treatment groups (Mann-Whitney test).

Antitumor immunity in a humanized MISTRG neuroblastoma model. (G) Cytotoxic activity of NK cells from two allogeneic donors were tested against K562 cells. Media were supplemented with MISTRG, NSG, human $\mathrm{AB}^{+}$serum, or matched heparinized plasma. Tumor lysis is significantly worse with the addition of MISTRG serum in resting (both) and IL-2-preactivated NK cells (donor 12). NK cells from donor 11 also have attenuated cytotoxicity with NSG serum. AB, antibody; IL, interleukin; log, logarithmic; NK, natural killer. 
A

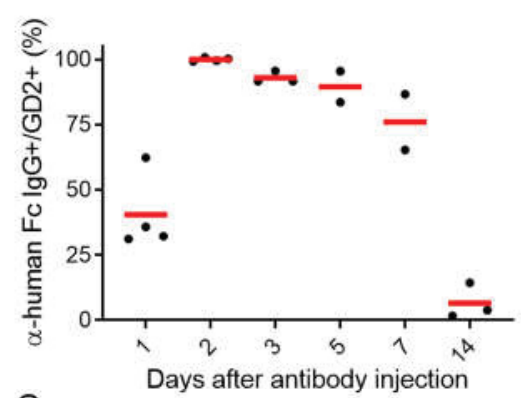

C

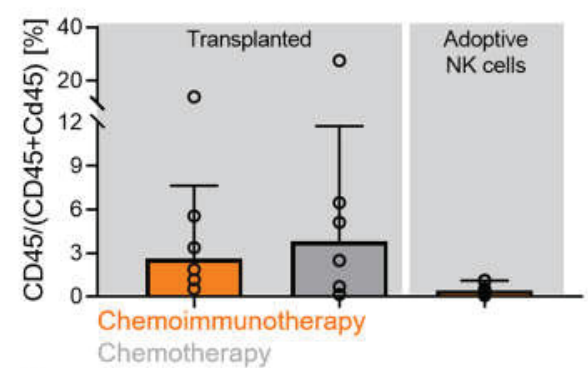

Chemotherapy

E
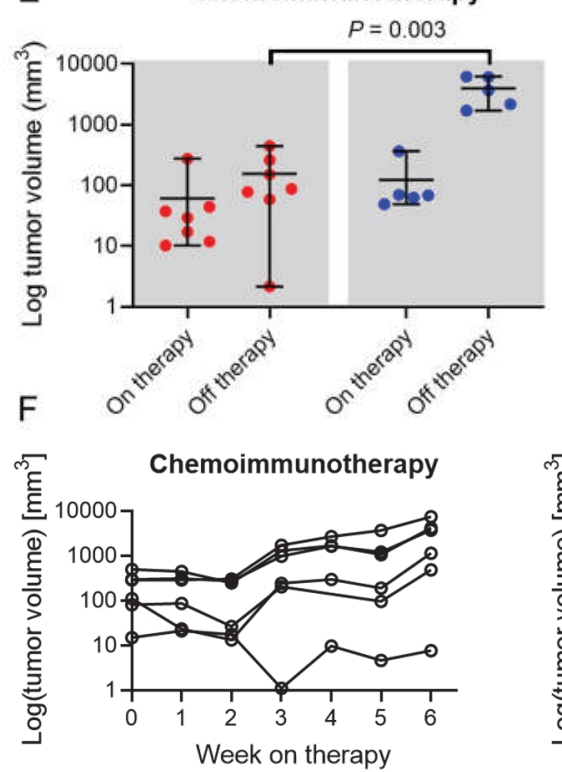

G

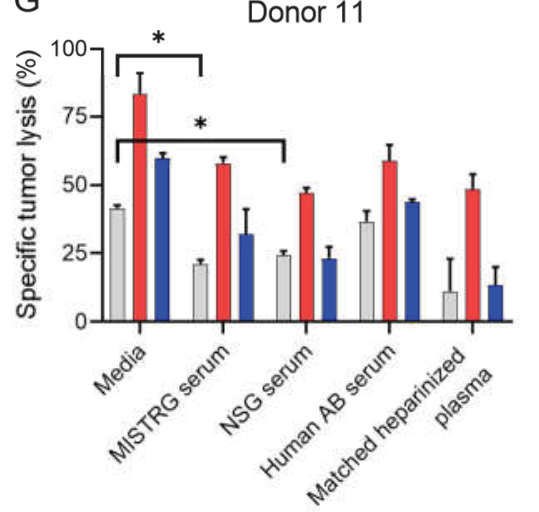

B

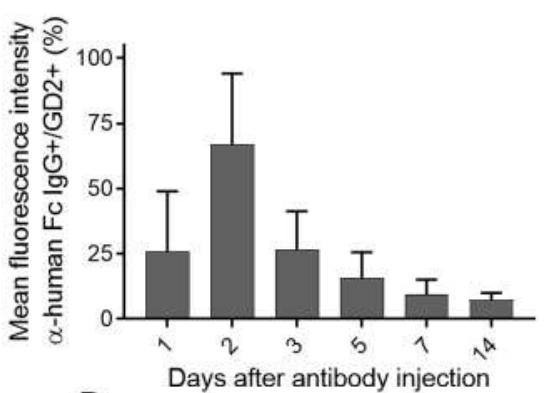

D

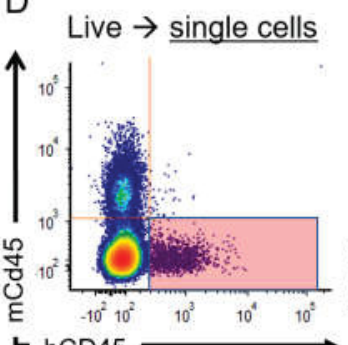

hCD45

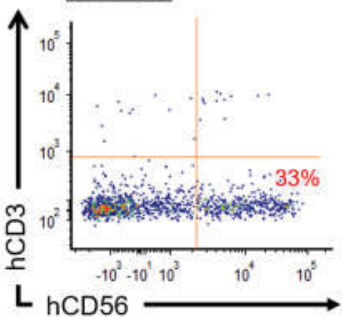

Chemotherapy
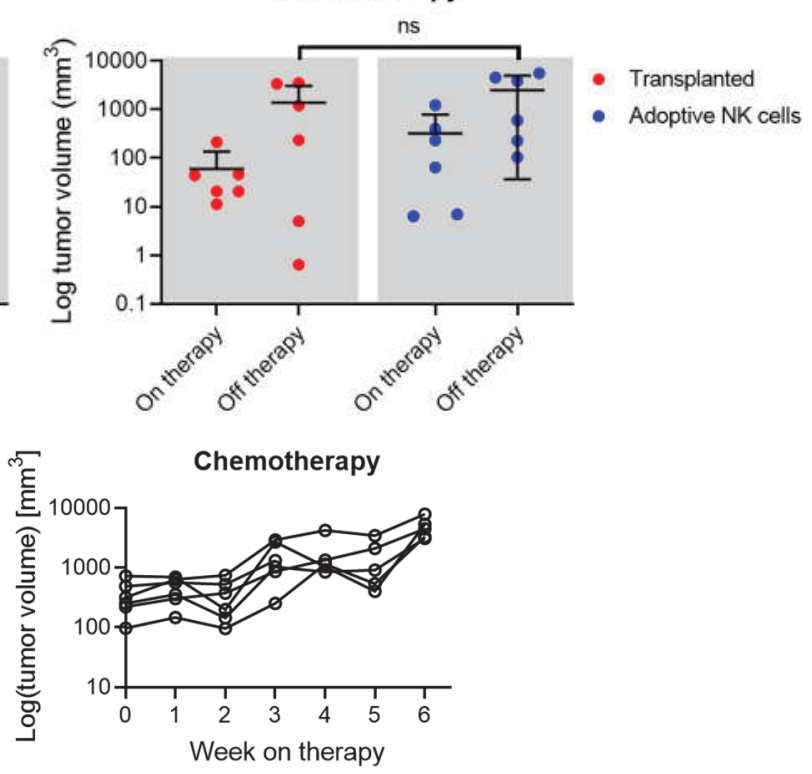

Donor 12

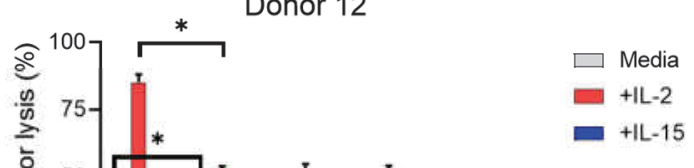


Despite the presence of anti-GD2 antibodies and IL-2, it is unclear what environmental pressures NK cells are exposed to within the mouse and in the tumor microenvironment. To assess whether these cells are generally suppressed by soluble factors in mouse serum, I tested the cytotoxic activity of two NK cell donors with K562 cells ex vivo. I supplemented the culture media with $33 \%$ of serum from $\mathrm{AB}^{+}$humans and NSG or MISTRG mice. Donor-matched heparinized plasma was used as positive control for suppressed cytotoxicity [156]. Surprisingly, resting NK cells eliminated significantly less K562 tumor cells when exposed to MISTRG $(P=0.04$ [donor 8]; $P=$ 0.02 [donor 12]) and NSG serum $(P=0.034$ [donor 11]). This suppressive effect was not overcome by IL-2-preactivated NK cells in one donor $(P=0.023$ [donor 11]) and implies that mouse serum exert suppressive effects on NK cells that develop in a human environment and render them ineffective after adoptive transfer into mice. Therefore, these findings may account for the lack of a robust antitumor effect with adoptively transferred NK cells in my in vivo experiments and warrant further investigation.

\section{Objective 3}

\section{A phase II clinical trial of adoptive transfer of haploidentical natural killer cells for consolidation therapy of pediatric AML}

Consolidation therapies for children with intermediate- or high-risk AML are urgently needed to achieve higher cure rates while limiting therapy-related toxicities. In this study, it was determined if adoptive transfer of NK cells from haploidentical KIRHLA-mismatched donors may prolong event-free survival in children with intermediaterisk AML who were in first complete remission after chemotherapy. Patients received cyclophosphamide (Day -7), fludarabine (Days -6 through -2), and subcutaneous IL-2 (Days $-1,1,3,5,7$, and 9). Purified, unmanipulated NK cells were infused on Day 0, and NK cell chimerism and phenotyping from peripheral blood were performed on Days 7 , 14, 21, and 28. The therapy schema is outlined in Figure 3-15. As primary endpoint, the event-free survival was compared to a cohort of 55 patients who completed chemotherapy on this trial and were in first complete remission but did not receive NK cells. Donor NK cell kinetics were determined as secondary endpoints.

Clinical characteristics of study cohort. Twenty-one patients (11 males [52\%]) with a median age of 6.0 years (range, $0.1-15.3$ years) at diagnosis received NK cell therapy. Sixteen (76\%) patients completed four courses and $5(24 \%)$ completed five courses of chemotherapy. All patients were negative for minimal residual disease (defined by $<0.1 \%$ leukemia cells) at the time of NK cell infusion. Patient demographic, hematologic, and engraftment characteristics are summarized in Table 3-1. The median duration of time from day 1 of the last cycle of chemotherapy to infusion of NK cells was 53 days (range, 31-111 days) and 10 days (range, 3-57 days) from the previous bone marrow aspirate obtained after completion of chemotherapy. The patients received a median of $12.5 \times 10^{6} \mathrm{NK}$ cells $/ \mathrm{kg}$ (range, 3.6-62.2 $\times 10^{6}$ cells $/ \mathrm{kg}$ ). Every patient tolerated and completed the preparative regimen and NK cell infusion with only 1 nonhematologic grade 3 adverse event (extravasation skin injury from cyclophosphamide). Of the 21 


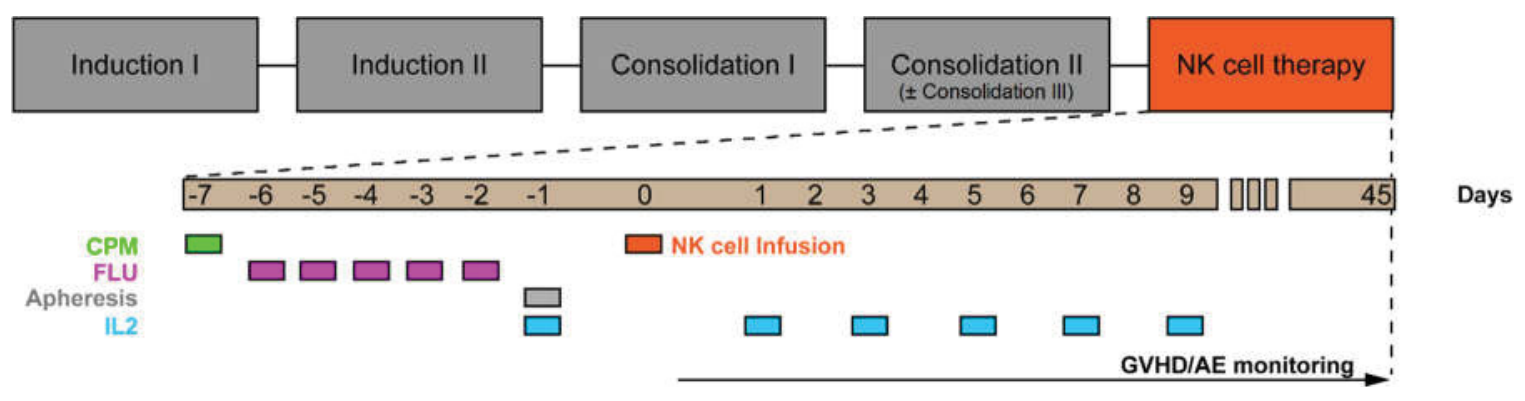

Figure 3-15. Therapy regimen of patients with AML

Notes: Therapy schema and time line of AML trial. AE, adverse events; CPM, cyclophosphamide; FLU, fludarabine. 
Table 3-1. Clinical characteristics of patients who received NK cell therapy

\begin{tabular}{|c|c|c|c|c|c|c|c|c|c|}
\hline UPN & $\begin{array}{c}\text { Age } \\
\text { (years) }\end{array}$ & Sex & $\mathrm{Bw}$ & $\mathrm{C}$ & KIR-HLA mismatch & $\begin{array}{c}\text { Peak } \\
\text { chimerism \% }\end{array}$ & Day & $\begin{array}{c}\text { NK cells } \\
\text { dose } \\
\left(10^{6} / \mathrm{kg}\right)\end{array}$ & $\begin{array}{c}\text { Days from NK } \\
\text { cell transplant } \\
\text { to relapse }\end{array}$ \\
\hline 1 & 1.1 & $\mathrm{M}$ & $6 / 6$ & $\mathrm{C} 1 / \mathrm{C} 1$ & CD158a, CD158e1 & 0 & 7 & 16.5 & - \\
\hline 2 & 1.6 & $\mathrm{~F}$ & $4 / 6$ & $\mathrm{C} 2 / \mathrm{C} 2$ & CD158b & 1 & 14 & 11.5 & 629 \\
\hline 3 & 2.1 & M & $4 / 6$ & $\mathrm{C} 2 / \mathrm{C} 2$ & CD158b & 2 & 6 & 12.4 & - \\
\hline 4 & 10.2 & $\mathrm{~F}$ & $4 / 6$ & $\mathrm{C} 2 / \mathrm{C} 2$ & CD158b & 4 & 12 & 8.2 & 527 \\
\hline 5 & 1.2 & $\mathrm{~F}$ & $4 / 6$ & $\mathrm{C} 1 / \mathrm{C} 1$ & CD158a & 23 & 7 & 46.9 & 617 \\
\hline 6 & 15.4 & $\mathrm{~F}$ & $6 / 6$ & $\mathrm{C} 1 / \mathrm{C} 2$ & CD158e1 & 8 & 13 & 3.6 & - \\
\hline 7 & 1.2 & $\mathrm{~F}$ & $4 / 6$ & $\mathrm{C} 2 / \mathrm{C} 2$ & CD158b & 2 & 8 & 36.9 & - \\
\hline 8 & 0.1 & $\mathrm{~F}$ & $6 / 6$ & $\mathrm{C} 1 / \mathrm{C} 2$ & CD158e1 & 43 & 14 & 44.3 & - \\
\hline 9 & 0.5 & $\mathrm{~F}$ & $4 / 6$ & $\mathrm{C} 1 / \mathrm{C} 1$ & CD158a & 15 & 7 & 26.8 & - \\
\hline 10 & 4.5 & M & $6 / 6$ & $\mathrm{C} 1 / \mathrm{C} 1$ & CD158a, CD158e 1 & 6 & 7 & 14.8 & $300^{\dagger}$ \\
\hline 11 & 2.1 & M & $6 / 6$ & $\mathrm{C} 1 / \mathrm{C} 1$ & CD158a, CD158e 1 & 2 & 7 & 25.7 & - \\
\hline 12 & 13.2 & $\mathrm{~F}$ & $6 / 6$ & $\mathrm{C} 1 / \mathrm{C} 1$ & CD158a, CD158e 1 & 15 & 7 & 6.7 & 228 \\
\hline 13 & 15.3 & $\mathrm{~F}$ & $4 / 6$ & $\mathrm{C} 1 / \mathrm{C} 1$ & CD158a & 14 & 12 & 5.2 & - \\
\hline 14 & 12.4 & M & $4 / 6$ & $\mathrm{C} 1 / \mathrm{C} 1$ & CD158a & 3 & 7 & 8.8 & $186^{\dagger}$ \\
\hline 15 & 1.6 & M & $4 / 6$ & $\mathrm{C} 2 / \mathrm{C} 2$ & CD158b & 0 & 7 & 12.5 & - \\
\hline 16 & 14.3 & $\mathrm{~F}$ & $4 / 6$ & $\mathrm{C} 1 / \mathrm{C} 1$ & CD158a & 22 & 7 & 5.7 & $231^{\dagger}$ \\
\hline 17 & 0.7 & M & $4 / 6$ & $\mathrm{C} 2 / \mathrm{C} 2$ & CD158b & 32 & 21 & 42.4 & - \\
\hline 18 & 3.0 & M & $4 / 6$ & $\mathrm{C} 1 / \mathrm{C} 1$ & CD158a & 0 & 7 & 62.6 & - \\
\hline 19 & 4.0 & M & $4 / 6$ & $\mathrm{C} 1 / \mathrm{C} 1$ & CD158a & 4 & 7 & 12.4 & - \\
\hline 20 & 12.2 & M & $6 / 6$ & $\mathrm{C} 1 / \mathrm{C} 2$ & CD158e1 & 1 & 7 & 9.0 & 367 \\
\hline 21 & 9.2 & M & $6 / 6$ & $\mathrm{C} 1 / \mathrm{C} 1$ & CD158a, CD158e1 & 10 & 7 & 19.3 & - \\
\hline
\end{tabular}

Notes: CNS, central nervous system; HLA, human leukocyte antigen; KIR, killer immunoglobulin-like receptor; N/A, not available; NK, natural killer; UPN, unique patient number. $\dagger$ Deceased. 
patients treated, 20 (95\%) developed $\geq$ grade 3 neutropenia, and 5 (24\%) developed grade $\geq 3$ thrombocytopenia. All patients but one had absolute neutrophil (median time to recovery, 13 days [7-45]) and platelet count recovery (median time to recovery, 33 days [9-52]) within 4 five days after NK cell infusion and none had opportunistic infections or notable bleeding. Graft-versus-host disease did not occur in any patients.

NK cell kinetics after adoptive transfer. On Day 7 after NK cell infusion, the median absolute blood NK cell count was 112 cells/ $\mu \mathrm{L}$ (range, 57-454 cells $/ \mu \mathrm{L}$ ), which remained stable with a median of 148 cells $/ \mu \mathrm{L}$ (range, $47-382$ cells $/ \mu \mathrm{L}$ ) on Day 14,113 cells $/ \mu \mathrm{L}$ on Day 21 (range, $53-382$ cells $/ \mu \mathrm{L}$ ), and 120 cells $/ \mu \mathrm{L}$ on Day 28 (range, $61-562$ cells $/ \mu \mathrm{L})$. Although white blood cell counts continuously increased after NK cell infusion $(P=.038)$, the NK cell numbers remained stable during that period $(P>.05)$. The average number of alloreactive NK cells increased from 41 cells $/ \mu \mathrm{L}$ (range, $0-180$ cells $/ \mu \mathrm{L}$ ) on Day 7 to 53 cells $/ \mu \mathrm{L}$ (range, $2-274$ cells $/ \mu \mathrm{L}$ ) on Day 14,57 cells $/ \mu \mathrm{L}$ (range, $5-246$ cells $/ \mu \mathrm{L}$ ) on Day 21 , and 79 cells $/ \mu \mathrm{L}$ (range, $0-306$ cells $/ \mu \mathrm{L} ; P=.032$ ) on Day 28. The fraction of KIR-HLA-mismatched, alloreactive NK cells expanded in 17 patients and contracted in four patients. Donor NK cell chimerism was detected in a total of 18 patients, four of whom were noted to have rising NK cell levels over time. The median peak donor NK cell chimerism was 4\% (range, $0 \%-43 \%$ ). Eleven of 18 patients $(61 \%)$ had persistent levels above the threshold of $1 \%$ at four weeks after NK cell infusion (median, 2\%; range, 1\%-30\%). The level of alloreactive NK cells in the peripheral blood or donor NK cell chimerism was not correlated with the infused NK cell dose $(P>.05)$. These results are shown in Figure 3-16.

Survival analysis. Basic demographic and hematologic characteristics of the study cohort and controls, such as age, gender, CNS status, and French-British-American (FAB) classification, were not significantly different $(P>0.05)$. Of the eight patients $(38 \%)$ who experienced relapse after adoptive transfer of NK cells, $3(14 \%)$ died of the disease. The median follow-up time for the entire cohort was 1,698 days, and relapse occurred between 186-629 days after NK cell infusion. NK cell infusion did not improve the cumulative incidence of relapse $(0.393$ [95\% confidence interval: $0.182-0.599$ ] vs. 0.35 [0.209-0.495]; $P=.556)$, event-free survival $(60.7 \pm 10.9 \%$ vs. $69.1 \pm 6.8 \% ; P=.553)$ or overall survival $(84.2 \pm 8.5 \%$ vs. $79.1 \pm 6.6 \% ; P=.663)$ compared to chemotherapy alone as demonstrated in Figure 3-17. Calculations indicated that survival distributions would have been statistically significantly different between the treated group and the control group if all patients in the treated group had been free of an event (100\% vs 69.1\%). Event-free survival but not overall survival was associated with the expression of CD158a $(P=.028), \operatorname{CD} 158 b(P=.021)$, and NKG2D $(P=.017)$, as well as with the NK cell count $(P=.007)$ on Day 7 . The number of alloreactive NK cells was not associated with event-free or overall survival at any time point. Most children with disease relapse received less than the average phase II NK cell dose which is shown in Figure 3-17. Therefore, a lower cell dose could be responsible for negative outcomes in this study. In this figure, six patients from the phase I cohort with intermediate-risk AML are included [69]. They received an average of $29 \times 10^{6} \mathrm{NK}$ cells/kg and only one patient with $+21 \mathrm{M} 1$ AML received $7.3 \mathrm{NK}$ cells $/ \mathrm{kg}$ which is below the average phase II cell dose. 

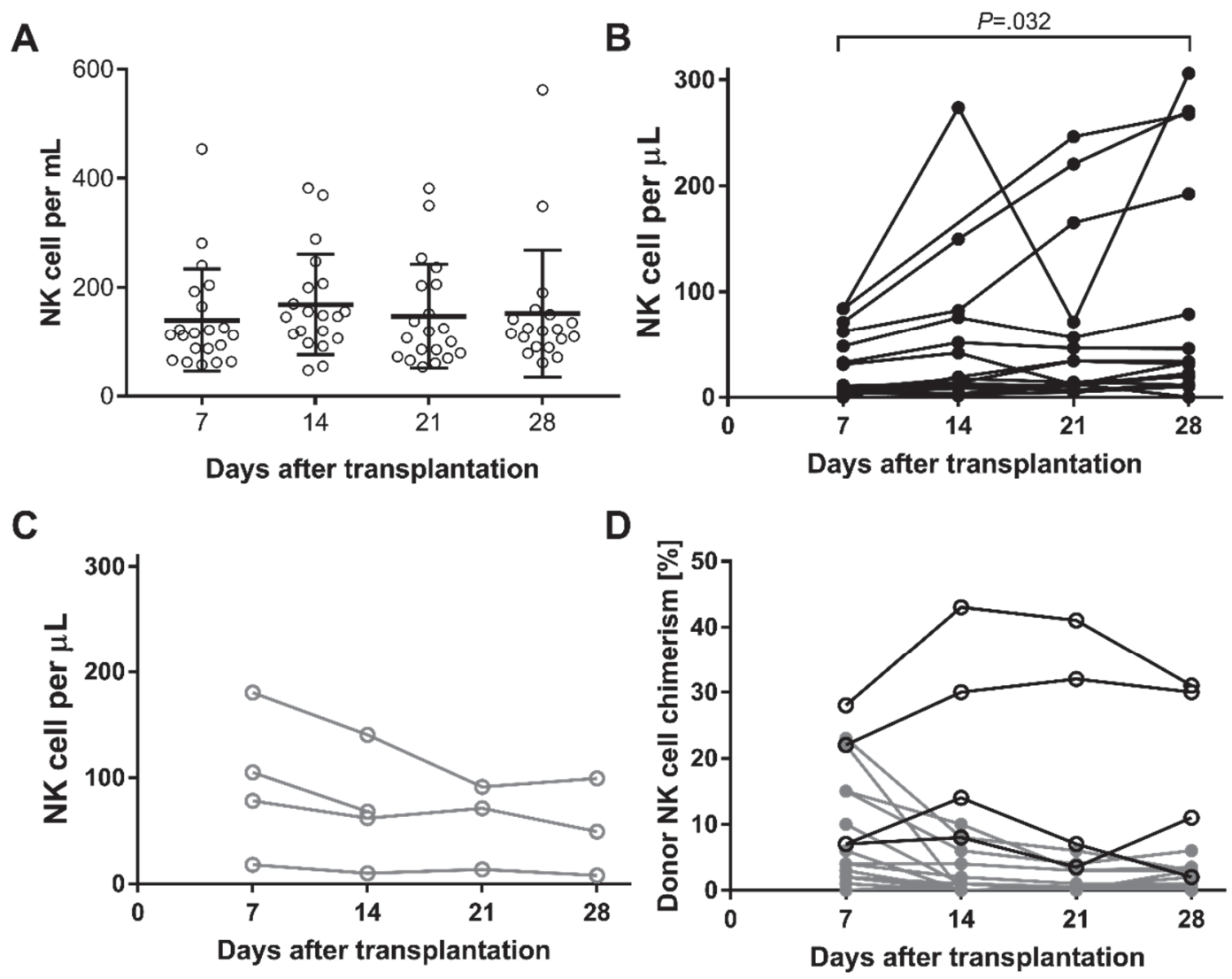

Figure 3-16. NK cell kinetics in patients after adoptive transfer of haploidentical KIR-HLA-mismatched NK cells

Notes: Average NK cell engraftment (A) in peripheral blood is plotted over time. KIRHLA-mismatched NK cells expanded over time in 17 patients (B) and contracted in four patients (C). Donor NK cell chimerism was detected in 18 patients (D, gray line), of which 4 were noted to have rising levels over time (black line). 
A

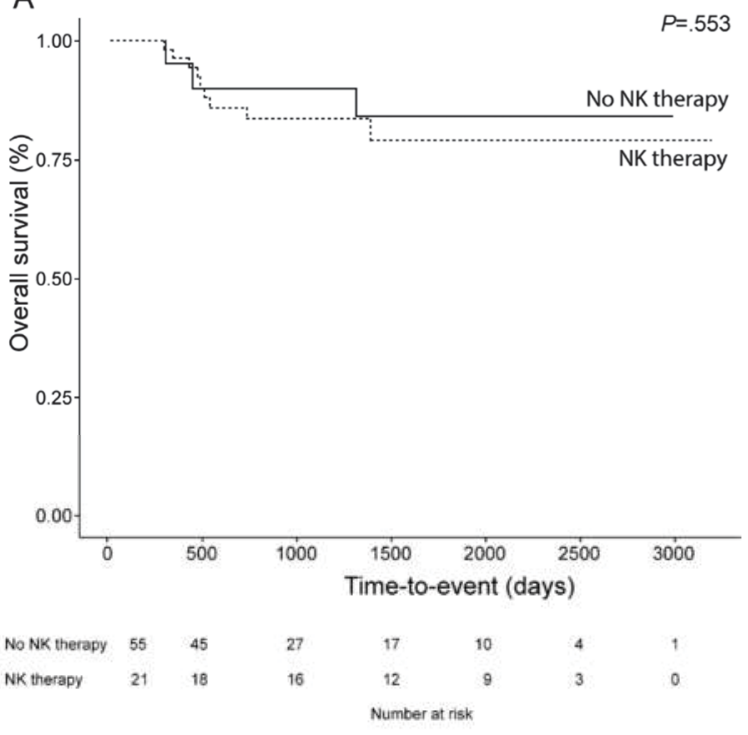

B

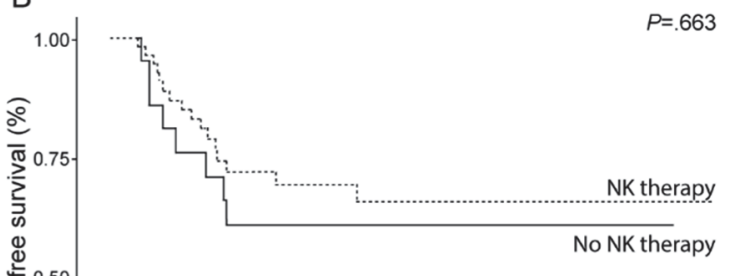

$\begin{array}{lrrrrrrr}\text { No NK therapy } & 55 & 40 & 22 & 16 & 9 & 4 & 1 \\ \text { NK therapy } & 21 & 15 & 11 & 9 & 6 & 2 & 0\end{array}$

Number at risk

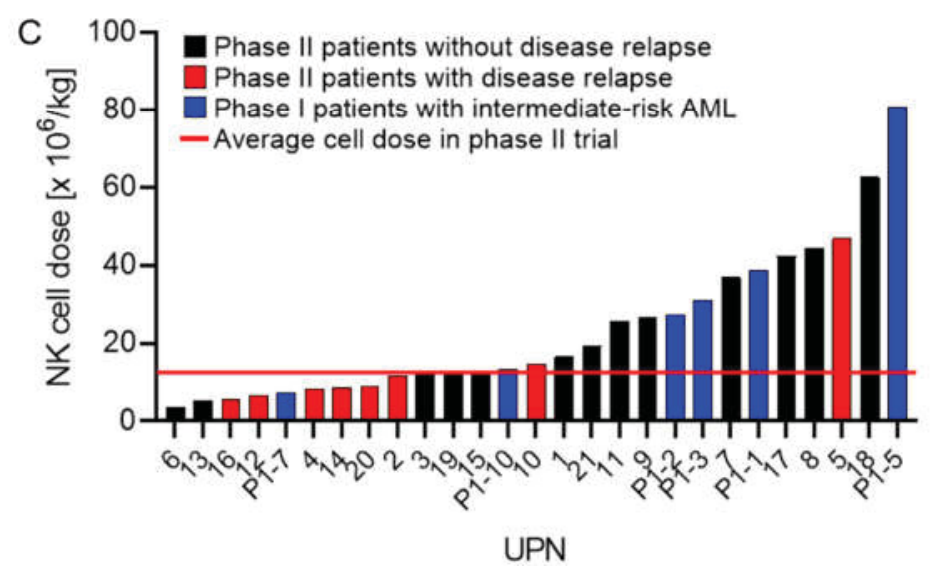

Figure 3-17. Survival curves and NK cell dose distribution

Notes: Kaplan-Meier survival curves comparing the overall survival (A) and event-free survival (B) of 21 patients with NK cell infusion (dashed line) with that of 53 patients who completed four or five courses of chemotherapy but did not receive NK cell infusion (solid line). (C) Patients are plotted by ascending order of infused NK cell dose per kg. Six of $12(50 \%)$ patients who received NK cells at a lower than the average phase II NK cell dose (red line) experienced disease relapse (red bar). 


\section{Analysis of NK cell phenotype and reconstitution in children treated with combined chemoimmunotherapy for neuroblastoma}

NK cells are the primary effector cells in ADCC in neuroblastoma. Specifically, the abundant population of CD56 ${ }^{\mathrm{dim}} \mathrm{NK}$ cells highly expresses the low-affinity receptor $\mathrm{CD} 16$ that recognizes the $\mathrm{F}_{\mathrm{c}}$ fragment of $\operatorname{IgG}$ and is required for $\mathrm{ADCC}[2,3]$. However, NK cells are also able to mediate antitumor effects through complex interactions between activating and inhibitory NK cell surface receptors and their cognate ligands expressed on target cells [26]. Ongoing research aims to better understand the quantitative and qualitative differences in NK cells that mitigate the clinical efficacy of monoclonal antibody therapy and may explain the variability of clinical responses to immunotherapy. The presence of certain Fc $\gamma \mathrm{R}$ polymorphisms $[157,158]$ that affect the affinity between CD16 and the $F_{c}$ fragment or specific KIR-HLA associations [159, 160] modulates clinical outcomes in patients with neuroblastoma undergoing immunotherapy. Therefore, a detailed longitudinal characterization of endogenous NK cells isolated from affected patients may broaden our current knowledge of NK cell biology in the context of neuroblastoma.

In this study, NK cells were analyzed from patients with newly-diagnosed, highrisk neuroblastoma who enrolled in the phase II trial (NCT01857934) at St. Jude Children's Research Hospital. Children received induction chemotherapy identical to that described in the ANBL0532 trial [91] combined with intravenously administered hu14.18K322A (days 2-5), subcutaneously administered low-dose IL-2 (days 6, 8, 10, 12,14 , and 16), and daily subcutaneous GM-CSF (day 7 until neutrophil count $\geq 2,000$ $\mathrm{mm} 3$ ). The consolidation regimen is reported elsewhere [93] and consists of myeloablative busulfan and melphalan, followed by autologous hematopoietic progenitor cell infusion. During consolidation, consenting patients received haploidentical CD56 ${ }^{+}$ $\mathrm{CD}^{-}$NK cells purified in a two-step procedure with the CliniMACS system (Miltenyi Biotec) [69]. NK cells were infused any time between day 2-5 after autologous hematopoietic progenitor cell infusion and four additional daily doses of hu14.18K $322 \mathrm{~A}$ and IL-2 and daily intravenous GM-CSF (beginning day 10 after NK cell infusion until neutrophil count $\geq 2,000 \mathrm{~mm} 3$ ) were given. Maintenance therapy was administered as described in the ANBL0032 trial with hu14.18K322A substituting ch14.18 [80]. The therapy schema is outlined in Figure 3-18.

Clinical characteristics of study cohort. Forty-six patients (25 [54\%] males) with a median age of 2.8 years $(0.5-15.2$ years) were included. Patient demographic, disease-specific, and therapy-related information are summarized in Table 3-2. Forty-two (91\%) patients received autologous bone marrow transplantation. Of which, 30 (71\%) received haploidentical NK cells (median cell dose, $22.6[3.0-114.0] \times 10^{6} / \mathrm{kg}$ ). Four patients did not undergo autologous bone marrow transplantation due to persistent infection $(\mathrm{N}=2)$ or parental refusal $(\mathrm{N}=2)$. At the time of analysis, five patients $(11 \%)$ had died of their disease.

NK cell kinetics during chemoimmunotherapy. The kinetics of $\mathrm{CD} 16^{+}$and total NK cell counts in the peripheral blood followed similar trends during induction 


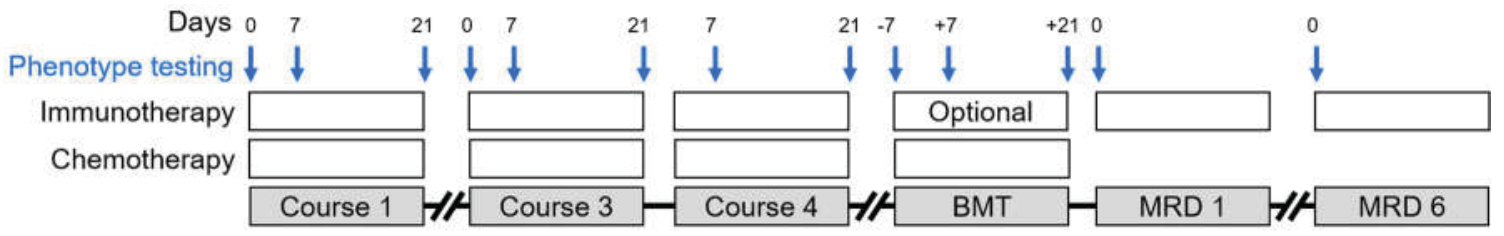

Figure 3-18. Therapy regimen of patients with high-risk neuroblastoma and time points of phenotype testing

Notes: Therapy schema of neuroblastoma trial and time points when peripheral blood samples were obtained for NK cell phenotyping. BMT: bone marrow transplantation; MRD: minimal residual disease therapy phase. 
Table 3-2. Clinical characteristics of studied patient cohort

\begin{tabular}{|c|c|}
\hline Demographic and clinical summary & Patient rates $(N=46)$ \\
\hline \multicolumn{2}{|l|}{ Age at diagnosis, $\mathrm{y}$} \\
\hline Median & 2.8 \\
\hline Range & $0.5-15.2$ \\
\hline \multicolumn{2}{|l|}{$\operatorname{Sex}(\%)$} \\
\hline Female & $21(45.7)$ \\
\hline Male & $25(54.3)$ \\
\hline \multicolumn{2}{|l|}{ Race (\%) } \\
\hline White & $31(67.4)$ \\
\hline African American & $12(26.1)$ \\
\hline Other & $3(6.5)$ \\
\hline \multicolumn{2}{|l|}{ INSS stage $(\%)$} \\
\hline $2 \mathrm{~B}$ & $1(2.2)$ \\
\hline 3 & $7(15.2)$ \\
\hline 4 & $38(82.6)$ \\
\hline \multicolumn{2}{|l|}{$M Y C N$ status (\%) } \\
\hline Not amplified & $26(56.5)$ \\
\hline Amplified & $19(41.3)$ \\
\hline Unknown & $1(2.2)$ \\
\hline \multicolumn{2}{|l|}{ Shimada histology (\%) } \\
\hline Favorable & $2(4.3)$ \\
\hline Unfavorable & $32(69.6)$ \\
\hline Unknown & $12(26.1)$ \\
\hline \multicolumn{2}{|l|}{ Survival status (\%) } \\
\hline Alive & $42(91.3)$ \\
\hline Deceased & $4(8.7)$ \\
\hline \multicolumn{2}{|l|}{ Autologous transplant (\%) } \\
\hline Yes & $42(91.3)$ \\
\hline No & $4(8.7)$ \\
\hline \multicolumn{2}{|c|}{$\begin{array}{l}\text { Haploidentical NK cell therapy as part of autologous transplant } \\
(\%)\end{array}$} \\
\hline Yes & $30(65.2)$ \\
\hline No & $16(34.8)$ \\
\hline \multicolumn{2}{|l|}{ Donor } \\
\hline Father & $16(53.3)$ \\
\hline Mother & $14(46.7)$ \\
\hline \multicolumn{2}{|c|}{ Infused NK cell dose, $\times 10^{6} / \mathrm{kg}$ median (range) } \\
\hline Median & 22.6 \\
\hline Range & $3.0-114.0$ \\
\hline \multicolumn{2}{|l|}{ Infused NKT cell dose, $\times 10^{6} / \mathrm{kg}$} \\
\hline Median & 0.01 \\
\hline Range & $0.00-0.10$ \\
\hline
\end{tabular}


Table 3-2. Continued

\begin{tabular}{ll}
\hline Demographic and clinical summary & Patient rates $(N=46)$ \\
\hline Infused T cell dose, $\times 10^{6} / \mathrm{kg}$ & 0.00 \\
Median & $0.00-0.01$ \\
Range & $16(53.3)$ \\
Donor HLA-patient KIR mismatch $(\%)$ & $14(46.7)$ \\
Yes & $12(40.0)$ \\
No & $1(3.3)$ \\
KIR2DL1 & $7(23.3)$ \\
KIR2DL2/3 & \\
KIR3DL1 & $15(50.0)$ \\
Autologous HLA-KIR mismatch $(\%)$ & $15(50.0)$ \\
Yes & \\
No & 21 \\
NK cell chimerism on day 7 after infusion & 5.9 \\
No. patients & $0.0-81.0$ \\
Median $(\%$ donor $)$ & \\
Range & 25 \\
NK cell chimerism on day 21 after infusion & 0.0 \\
No. patients & $0.0-35.0$ \\
Median (\% donor) &
\end{tabular}

Notes: HLA, human leukocyte antigen; KIR, killer-cell immunoglobulin-like receptor; INSS, International Neuroblastoma Staging System. 
therapy, with profound NK cell deficiency on day 7 of each course. Compared to pretreatment levels, the median NK cell counts decreased from $91(5-1,635)$ cells $/ \mu \mathrm{L}$ at baseline to $1(0-8)$ cell $/ \mu \mathrm{L}(\mathrm{P}<0.001)$ on day 7 of course $1,14(3-246)$ cells $/ \mu \mathrm{L}$ $(\mathrm{P}<0.001)$ on day 7 of course 3 , and $1(0-4)$ cel1 $/ \mu \mathrm{L}(\mathrm{P}<0.001)$ on day 7 of course 4 , respectively. By comparing the NK cell counts on day 7 among the three induction courses, NK cytopenia was less severe during course 3 (i.e., cisplatin and etoposide) than in the other two courses (i.e., cyclophosphamide/topotecan and cyclophosphamide/doxorubicin/vincristine $)(\mathrm{P}<0.001)$. By day 21 , the median NK cell counts fully reconstituted to equivalent (course 1 and 4 ) or higher levels than that at baseline (164 [32-845] cells $/ \mu \mathrm{L}$ on day 21 of course $4 ; \mathrm{P}=0.012)$. These results are shown in Figure 3-19. All NK cell receptors, including CD16, KIRs, NKG2D, and NKp46, shared similar longitudinal expression patterns during chemoimmunotherapy. The percentage of CD158a+ cells at baseline (12.2\% [6.1-31.0] vs. 7.7\% [0.8-31.3]; $\mathrm{P}=0.057)$ and NKp44+ cells on day 21 of course $1(12.1 \%[2.2-50.5]$ vs. $6.7 \%[0.4-$ 28.2]; $\mathrm{P}=0.098$ ) was marginally higher in those with stable disease than in responders.

\section{NK cell reconstitution during consolidation with haploidentical NK cell}

infusion. During consolidation, patients who received haploidentical NK cells had higher mean NK cell counts at day 7 after NK cell infusion than did those who did not receive NK cells $(11 \pm 10$ cells $/ \mu \mathrm{L}$ vs. $8 \pm 5$ cells $/ \mu \mathrm{L})$. On day 21 post transplant, this difference persisted $(182 \pm 138$ cells $/ \mu \mathrm{L}$ vs. $144 \pm 25$ cells $/ \mu \mathrm{L})$. The small sample size of patients with complete data set precluded statistical analysis. The total NK cell count on day 7 post transplant $(\mathrm{r}=0.39, \mathrm{P}=0.021)$ but not on day $21(\mathrm{r}=0.33, \mathrm{P}=0.087)$ correlated with the infused number of total nucleated cells. Median donor NK cell chimerisms were $6 \%$ $(0-81)$ on day 7 and $0 \%(0-35)$ on day 21 . Notably, all patients receiving more than 25 $\times 10^{6} / \mathrm{kg}$ NK cells had donor NK cells detectable in the peripheral blood seven to 21 days after infusion; however, all four patients with undetectable donor NK cells at any timepoint after NK cell infusion received less NK cells. All 11 of 30 (37\%) patients with evaluable alloreactive NK cell counts at days 7 and 21 post transplant had an expansion of this subpopulation, albeit at various degrees. When compared with patients who had less residual disease (i.e., responders after induction therapy), expansion of alloreactive NK cells was more notable in patients with more residual disease after induction therapy (i.e., stable disease) (median change, 8 [0.03-75] cells $/ \mu \mathrm{L}$ vs 73 [39-106] cells $/ \mu \mathrm{L}$ ). All seven patients who had received NK cells from CD158e1 (KIR3DL1)-mismatched donor are survivors, whereas 4 of those without CD158e1 (KIR3DL1)-mismatch had died. These results are shown in Figure 3-20.

NK cell reconstitution during maintenance therapy. During maintenance therapy, the median NK cell counts peaked at $143(23-511)$ cells $/ \mu \mathrm{L}(\mathrm{P}<0.001)$ prior to course 3 and returned to values comparable with that of pretherapy levels prior to course $6(\mathrm{P}>0.05)$. 


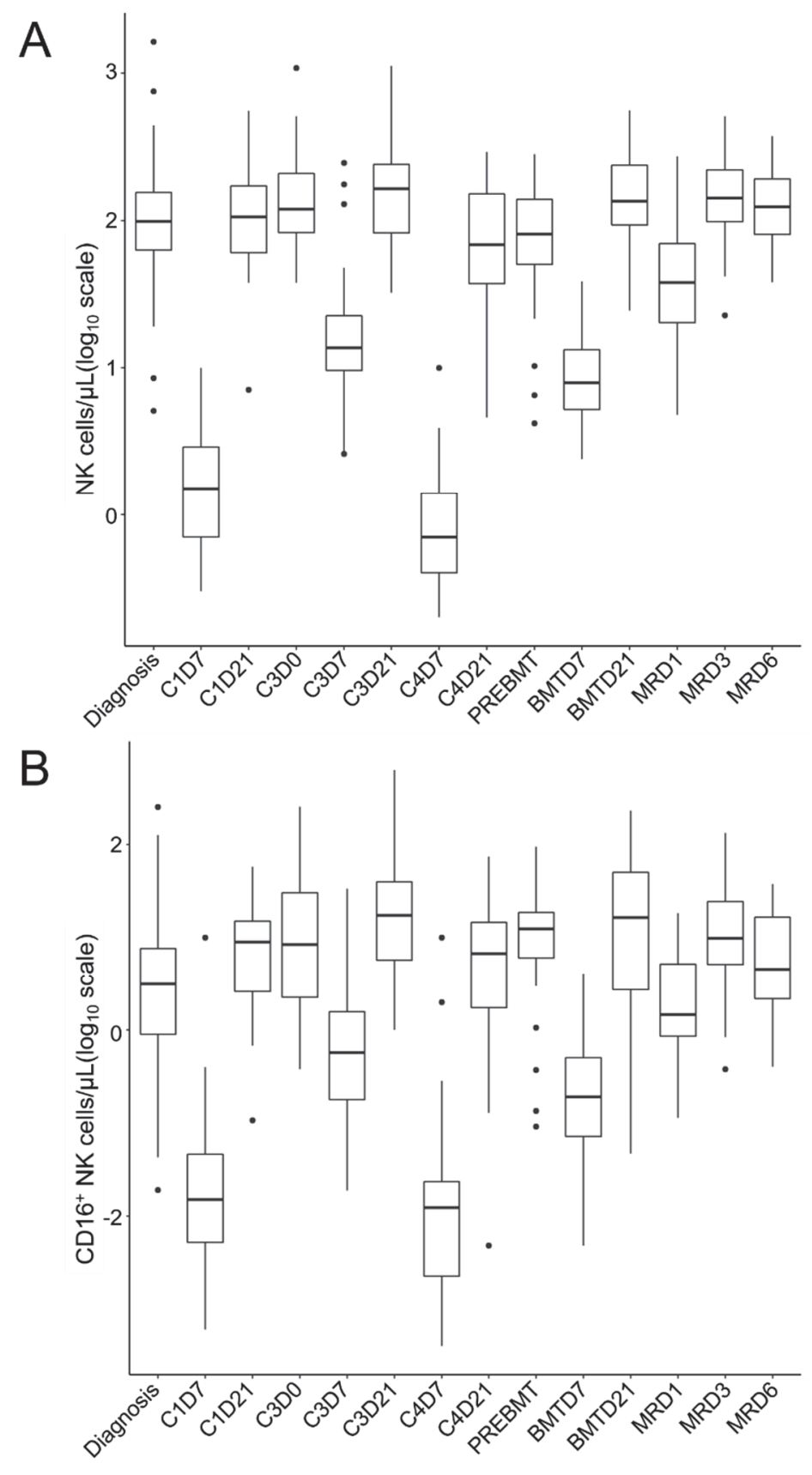

Figure 3-19. Box plot of total and $\mathrm{CD}^{+}{ }^{+} \mathrm{NK}$ cells (logarithmic scale) by time point

Notes: (A) Boxplots of longitudinal logarithmic NK cell count and (B) CD16 ${ }^{+} \mathrm{NK}$ cells per $\mu \mathrm{L}$ of peripheral blood in children with neuroblastoma undergoing chemoimmunotherapy. C1D7, course 1 day 7; C1D21, course 1 day 21; C3D0, course 3 day 0; C3D7, course 3 day 7; C3D21; course 3 day 21; C4D7, course 4 day 7; C4D21, course 4 day 21; PREBMT, prior to transplantation; BMTD, days after stem cell infusion; $\mathrm{MRD}$, minimal residual disease; NK, natural killer. 


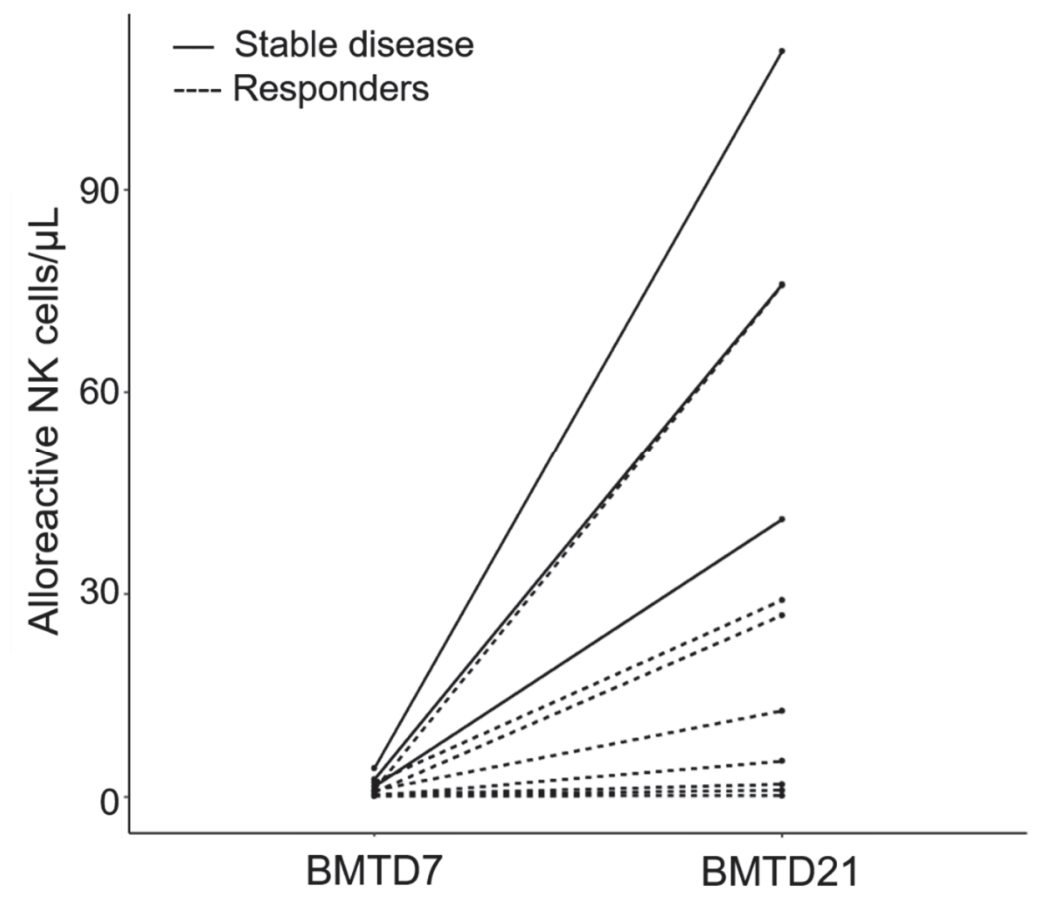

Figure 3-20. Individual alloreactive NK cell counts on days 7 and 21 after adoptive transfer of haploidentical and KIR-HLA-mismatched NK cells

Notes: Responders (complete, very good partial, partial response; $\mathrm{n}=8$ ) are shown as solid lines and those with stable disease $(n=3)$ as a dashed line. BMTD, Days after stem cell infusion; NK, natural killer. 


\section{NK cells from patients with neuroblastoma are dysfunctional}

NK cells are the primary effector cells in anti-GD2 antibody-induced ADCC in neuroblastoma. To characterize the cytotoxic activity of NK cells in children with newly diagnosed neuroblastoma, I purified NK cells from blood of 19 patients enrolled on our institutional phase II trial NB2012 (NCT01857934). NK cells were purified by negative selection and cultured for 12 to 18 hours in RPMI-based full medium in the presence or absence of cytokines (IL-2 or IL-15). I used those cells to perform a flow cytometrybased cytotoxicity assays with K562 cells. The effector-to-target (E:T) ratio was 5:1 with a 4-hour incubation period. Unstimulated patient-derived NK cells had significantly less cytotoxic activity against K562 cells than did NK cells from healthy adult controls $(P<$ 0.001; Figure 3-21); however, activation with IL-2 and IL-15 significantly increased the NK cell-mediated specific lysis of K562. NK cells are divided by the expression of the cell surface marker CD56 into cytokine producing CD56 $6^{\text {bright }}$ and cytotoxic CD56 ${ }^{\mathrm{dim}} \mathrm{NK}$ cells. Patients with neuroblastoma had a higher percentage population of resting CD56 ${ }^{\text {bright }} \mathrm{NK}$ cells than did adult controls. This percentage did not correlate with the age of the patient $(P>0.05)$. However, in patients and controls, the cytotoxic capacity ex vivo was inversely correlated with the percentage of CD56 bright $\mathrm{NK}$ cells $(\rho=-0.5 ; P=$ $0.003)$ and positively correlated with the NKp46 expression $(r=0.49 ; P=0.33)$, predominantly found in CD56 ${ }^{\mathrm{dim}} \mathrm{NK}$ cells. Early clinical tumor responses in the patients were defined as tumor volume reduction after two courses of induction chemoimmunotherapy that correlated with the ex vivo NK cell cytotoxicity measured at the time of diagnosis $(r=0.5 ; P=0.057)$.

To compare the tumor lysis by CD $56^{\text {bright }}$ to CD $56^{\mathrm{dim}} \mathrm{NK}$ cells, I purified NK cells from two donors and sorted the CD56 $6^{\mathrm{dim}}$ and CD56 $6^{\text {bright }}$ sub-populations. CD $56^{\mathrm{dim}} \mathrm{NK}$ cells eliminated $56 \pm 4 \%$ (donor 1 ) and $42 \pm 6 \%$ (donor 2 ) of K562 cells, and no lysis occurred with $\mathrm{CD} 56^{\text {bright }} \mathrm{NK}$ cells. Collectively, these findings indicate that NK cells from patients with neuroblastoma have inferior cytotoxicity against K562 than do those from healthy controls. Cytotoxicity is correlated with early clinical tumor response rates and may be determined by the amount of CD56 $6^{\text {bright }} \mathrm{NK}$ cells in the blood.

\section{Molecular analyses of NK cells derived from patients with neuroblastoma}

To determine the molecular profiles of the NK cells from patients with neuroblastoma, I applied a drop-seq single-cell RNA-seq approach. NK cells from patient samples were isolated via negative magnetic selection and used for the generation of gel bead-in-emulsion reaction vesicles and a single-cell barcoded library. Following sequencing, the data were analyzed. Targeting $10,000 \mathrm{NK}$ cells per sample, I analyzed a total of 33,359 peripheral blood NK cells from five patients with untreated neuroblastoma.

Unsupervised hierarchical clustering of combined patient NK cells identified two major NK cell clusters: CD56 ${ }^{\mathrm{dim}} \mathrm{NK} 1$ (larger cluster) and CD56 ${ }^{\text {bright }} \mathrm{NK} 2$ (smaller cluster) (Figure 3-22). The relative proportion of NK2 in patients 1, 2, and 3 accounted 

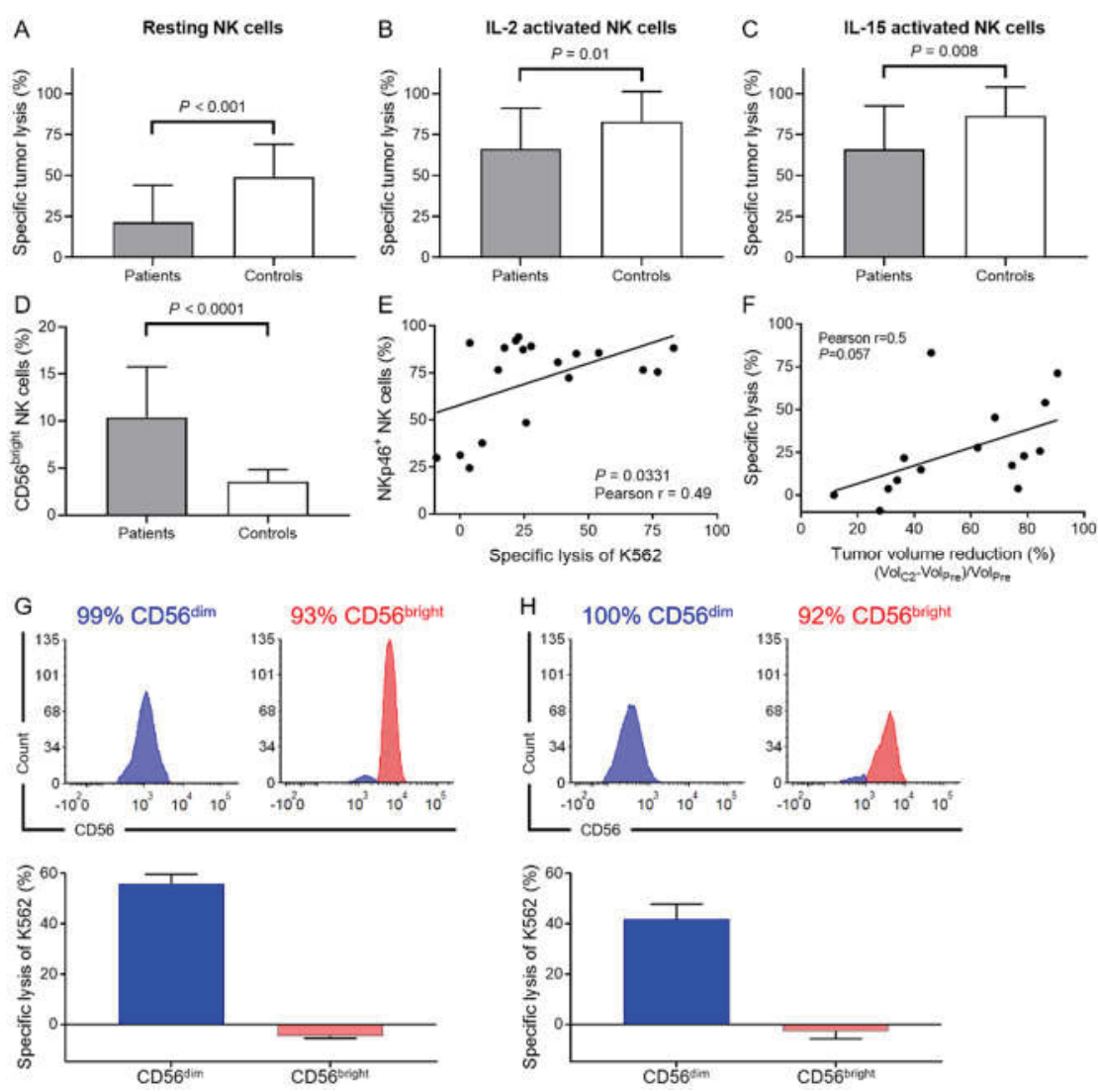

Figure 3-21. Ex vivo cytotoxic activity of NK cells from patients with neuroblastoma and healthy controls.

Notes: Ex vivo lysis of K562 cells by peripheral blood NK cells from 19 patients with newly diagnosed and untreated neuroblastoma (grey bar) is compared with those of 19 healthy adult controls (white bar). NK cells were either cultured for 12 to 18 hours in RPMI-based full medium in the (A) absence or presence of (B) IL-2 or (C) IL-15. NK cells from patients with neuroblastoma displayed decreased cytotoxicity against K562 cells and contained higher proportions of CD56 $6^{\text {bright }} \mathrm{NK}$ cells than did (D) NK cells from controls (unpaired t test or Mann-Whitney test). (E) Specific lysis correlates with NKp46 expression and (F) tumor volume reduction after two courses of therapy (early tumor response; Pearson correlation coefficient). CD56 ${ }^{\text {dim }}$ and $\mathrm{CD} 56^{\text {bright }} \mathrm{NK}$ cells were purified from $(\mathrm{G})$ donor 1 and $(\mathrm{H})$ donor 2. Histogram plots demonstrate the proportions of CD56 $6^{\text {dim }}$ (blue) or CD56 ${ }^{\text {bright }} \mathrm{NK}$ cells (red) that were used in the cytotoxicity assays with K562 cells. CD56 ${ }^{\mathrm{dim}} \mathrm{NK}$ cells eliminated $56 \pm 4 \%$ (donor 1 ) and $42 \pm 6 \%$ (donor 2 ) of K562 cells, and no lysis occurred with CD56 $6^{\text {bright }} \mathrm{NK}$ cells. 

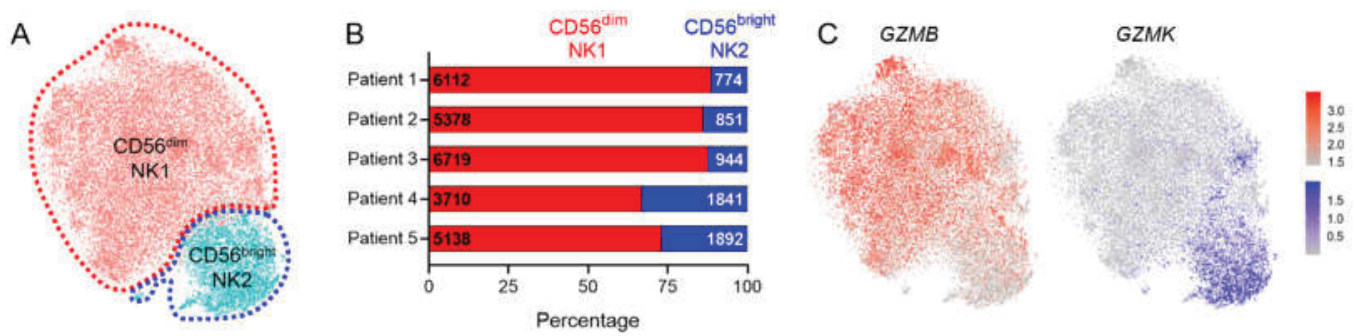

D
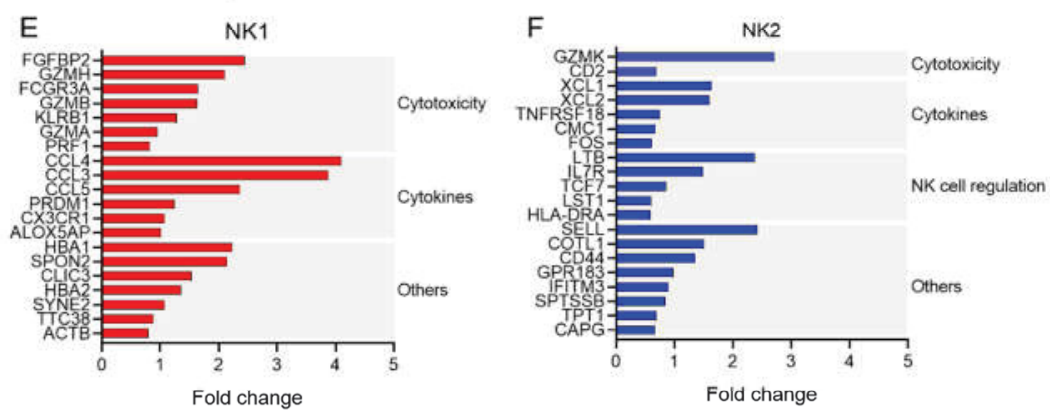

G
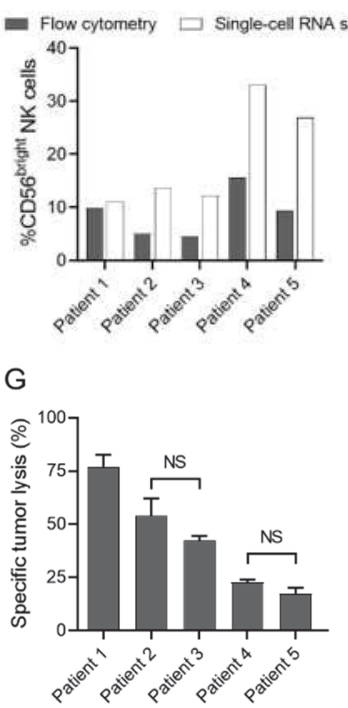

Figure 3-22. Single-cell transcriptome analysis of NK cells from patients with neuroblastoma

Notes: (A) Visualization of CD56dim NK1 and CD56bright NK2 cluster of combined peripheral blood NK cells from 5 patients with neuroblastoma using t-distributed stochastic neighbor embedding. (B) Relative percentages of CD56dim NK1 and CD56bright NK2 cells per patient. The absolute numbers of cells are plotted for each cluster. (C) Color-scaled expression of GZMB and GZMK. (D) Percentage of CD56bright NK cells identified by flow cytometry (grey bar) or single-cell RNA-seq (white bar). Top ten genes that are differentially expressed between the (E) NK1 and (F) NK2 subset and listed by function. (G) Lysis of K562 cells ex vivo by patient. 
for approximately $10 \%$ of all NK cells and was smaller than in patients 4 and 5, whose NK 2 cluster comprised more than $25 \%$ of all NK cells. To segregate the NK1 and NK2 cluster, I found $G Z M B$ to define the NK1 subset and GZMK for the NK2 subset. Identification of CD56 ${ }^{\text {bright }} \mathrm{NK} 2$ cells by flow cytometry and single-cell RNA-seq yielded similar results. When compared to the NK2 population, the NK1 cluster differentially expressed transcripts that encoded membrane-bound proteins (e.g., KLRBI and FCGR3A) and secreted proteins (e.g., $F G F B P 2, G Z M H$, and $G Z M B$ ) involved in cytotoxicity and pathogen defense, as well as proteins involved in chemotaxis (e.g., CCL3, CCL4, and $C C L 5$ ). Enriched transcripts in the NK2 cluster were associated with cytotoxicity (e.g., GZMK, CD2), lymphocyte chemotaxis and migration (e.g., XCL1 and XCL2) but also contained genes that regulate NK cell function (e.g., $L T B, I L 7 R$, and $T C F 7$ ). I found that the ex vivo cytotoxic capacity of each donor's NK cells correlated with the proportion of NK1 and inversely correlated with the proportion of NK2.

I repeated single-cell RNA-seq for four of the five patients after completion of two courses of chemoimmunotherapy and surgery, when tumor volumes were markedly decreased $\left(148 \pm 102 \mathrm{~mm}^{3}\right.$ versus $\left.14 \pm 14 \mathrm{~mm}^{3}\right)$. I aligned the patient single-cell RNAseq data obtained at $t_{2}$ (i.e., after two courses of treatment) and $t_{1}$ (i.e., before therapy) and found that the population density and distribution overlapped between both data sets (Figure 3-23). The relative proportion of NK1 to NK2 clusters remained stable at both time points in each patient, except in patient 2 who had an expansion of NK2 from 14\% at $t_{1}$ to $41 \%$ at $t_{2}$. This was also reflected by a shift in expression of GZMB and GZMK. I compared the differential gene expression at $t_{2}$ with $t_{1}$ for each patient individually. In patients 1,4 , and 5, most differentially expressed genes were downregulated and involved in cytokine production and NK cell regulation. The NK cells from patient 2 exhibited upregulation of the NK2-defining gene $G Z M K$, and concordantly, tumor lysis was diminished at $t_{2}$ than at $t_{1}$ for this patient. In the remaining three patients, no association was noted between the differential expression of the NK1- and NK2-defining genes or ex vivo cytotoxicity at $t_{2}$. 
Figure 3-23. Single-cell transcriptome analysis of NK cells from patients with neuroblastoma after therapy

Notes: (A) Aligned single-cell RNA-seq data sets of NK cells from patients with neuroblastoma before therapy $\left(t_{1}\right)$ and after two courses of treatment $\left(t_{2}\right)$. (B) Relative percentages of CD56 $6^{\text {dim }} \mathrm{NK} 1$ and CD $56^{\text {bright }} \mathrm{NK} 2$ cells per patient at $t_{1}$ and $t_{2}$. The absolute numbers of cells are plotted for each cluster. (C) Color-scaled expression of GZMB (red) and GZMK (blue) by patient and time point. The blue circle marks the NK2 population in patient 2 that expands after therapy. (D) Differential gene expression in NK cells of 4 patients at $t_{2}$ compared to $t_{1}$. Bars indicate average log fold change and are color coded to reflect association with gene function (striped bar: cytotoxicity [Tox]; black bar: cytokine production [Ck]; white bar: NK cell regulation [Reg]; grey bar: other [Oth]). (E) Lysis of K562 cells ex vivo by patient and time point. Light grey bars represent $t_{1}$ and dark grey bars represent $t_{2}$. 


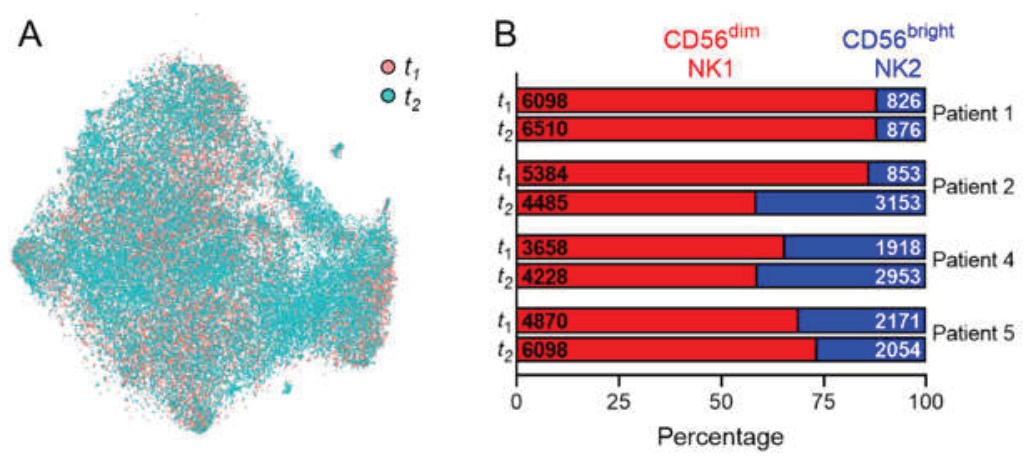

C Patient 1

Patient 2

Patient 4

Patient 5
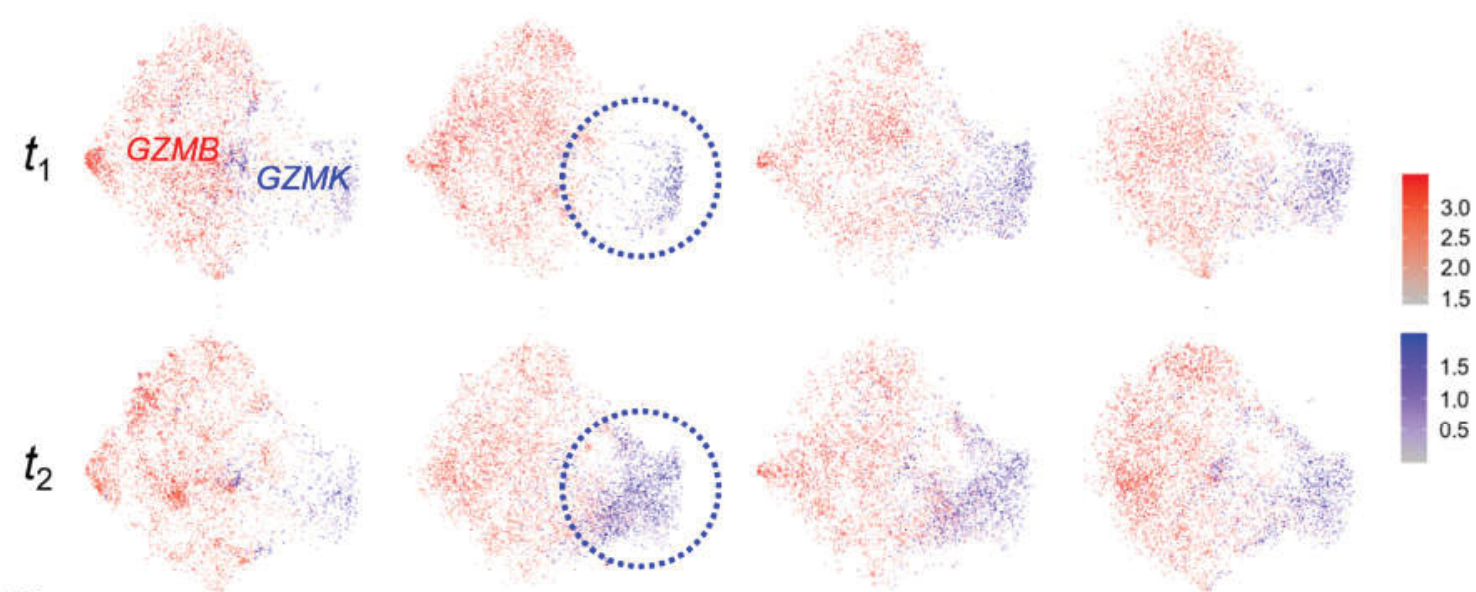

D

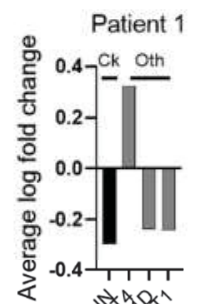

vinge
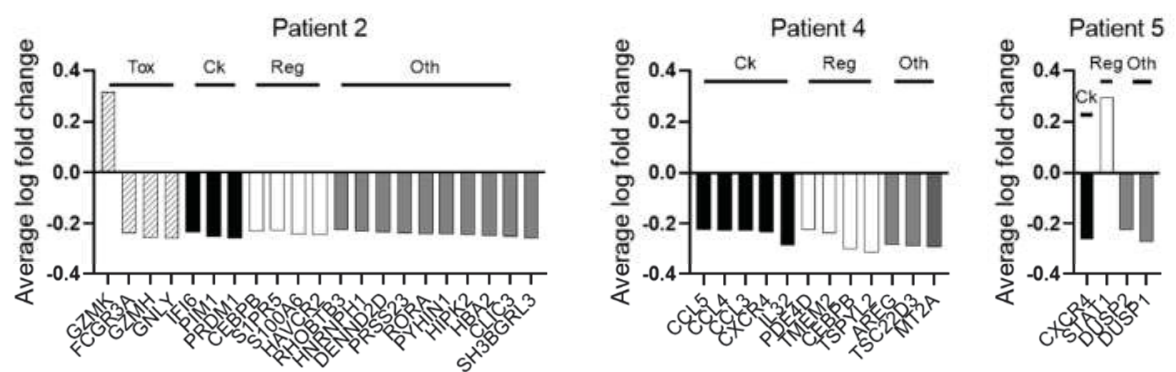

E

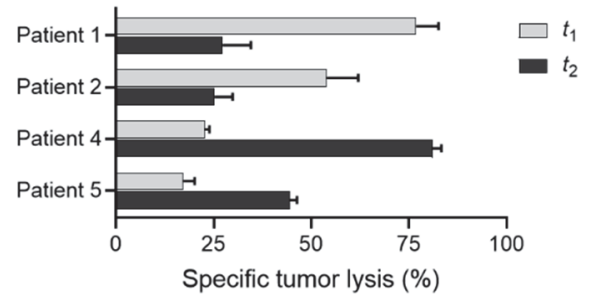




\section{CHAPTER 4. DISCUSSION}

\section{Objective 1}

In my dissertation research, I studied the contribution of IL-2 and IL-15, anti-GD2 antibody, ATRA, and HLA-KIR mismatch in NK cell-mediated cytotoxicity of neuroblastoma cells with the aim to better understand the cellular mechanisms of ADCC. I found that IL-2 improved ADCC in culture and induced equivalent cytotoxicity with lower antibody doses, supporting the continued use of this cytokine in neuroblastoma immunotherapy. In my experiments, I also demonstrated that the susceptibility of neuroblastoma cells to ADCC was improved after ATRA-induced neuronal differentiation which validates our current therapeutic approach in the clinic, where immunotherapy is administered with cis-retinoic acid. Although KIR-HLA mismatch was a strong signal for degranulation in culture, a larger proportion of NK cells degranulated with ADCC, presumably due to recruitment of KIR-HLA matched and CD16-expressing sub-groups. Currently, allogeneic KIR-HLA-mismatched NK cells are therapeutically administered to cancer patients in the clinic. However, my findings indicate that the tumor-directed effect could be further exerted when given with antibody. In this work, I found that IL-15, integrated in the current immunotherapy regimen for neuroblastoma, has significant preclinical anti-tumor activity in vitro and in vivo that exceeds the effect of IL-2. Based on these findings, I propose IL-15 as an IL-2 substitute and suggest as forthcoming steps the conduction of a larger preclinical trial to validate the results or clinical study to test the tolerability of IL-15 with immunotherapy in children with relapsed/refractory neuroblastoma and report the anti-tumor effect as a secondary endpoint. Together, the findings of this study highlight several potential ways to improve immunotherapy for patients with neuroblastoma and warrant further validation in vivo.

Many studies have described the enhancing effect of IL-2 on NK-cell growth and survival and suggested a supporting role in ADCC $[131,161,162]$. However, therapeutic administration of IL-2 has been limited in the clinic by the considerable toxicity associated with high doses [140]. For example, in the latest high-risk neuroblastoma study by the European International Society of Pediatric Oncology Neuroblastoma Group, $44 \%$ of patients who received high-dose recombinant IL-2 at $6 \times 10^{6} \mathrm{IU} / \mathrm{m}^{2}$ by subcutaneous injection were unable to tolerate the regimen and had to terminate their treatment prematurely [135]. The investigators of this study questioned the therapeutic role of rIL-2 in their trial, given that they did not find a survival benefit with the use of recombinant IL-2. However, the findings may have been confounded by the high dropout

\footnotetext{
${ }^{3}$ Portions of chapter from previously published article; final submission modified with permission of Springer Nature. Nguyen R, Houston J, Chan WK, et al. (2018) The role of interleukin-2, all-trans retinoic acid, and natural killer cells: surveillance mechanisms in anti-GD2 antibody therapy in neuroblastoma. Cancer Immunol Immunother, 67: 615-26 [134]. https://doi.org/10.1007/s00262-017-2108-6.

Portions of chapter from final submission modified with open access permission from Springer Nature. Nguyen R, Wu H, Pounds S, et al. (2019) A phase II clinical trial of adoptive transfer of haploidentical natural killer cells for consolidation therapy of pediatric acute myeloid leukemia. J. Immunother. Cancer. In press. Authors retain the copyright.
} 
rate of patients in the IL-2 treatment arm [135]. My dissertation research showed that IL2 improves ADCC in culture and therefore supports the continued use of IL-2 in neuroblastoma immunotherapy. The IL-2 concentration that was used is tolerable and physiologically achievable, falling within the range of serum levels from adult and pediatric pharmacokinetic studies $[140,163]$. Therefore, it can be argued that low doses of IL-2 may be an efficacious yet better tolerated option for children with neuroblastoma. ADCC is enhanced by greater IL-2 activation and anti-GD2 antibody concentration (of $>10 \mathrm{ng} / \mathrm{mL}$ ) until this effect reaches a plateau in vitro. In the future, several doses of Hu14.18K322A should be compared in combination with IL-2 in preclinical studies to determine which dose combination may yield the best anti-tumor effect. Clinically, dose de-escalation of anti-GD2 antibody therapy could result in better tolerability because it may decrease antibody-related pain, which is dose-dependent and currently managed in the clinic with preemptive administration of morphine and lowering of the anti-GD2 antibody infusion rates, extending up to 10 days (NCT01857934) [164].

For neuroblastoma, NK-cell therapy could be exploited as either part of anti-GD2 antibody therapy or cancer-directed immunosurveillance $[165,166]$, but the cellular mechanisms are less clearly understood in neuroblastoma than in AML [66]. In the present studies, ADCC was a strong signal for NK-cell degranulation. Therefore, adoptive transfer of allogeneic NK cells may be more effective when given with antibody. It was also observed that NK cells of all the tested donors express CD158b and were licensed and predominantly active, whereas other NK-cell populations exhibited less degranulation. CD158b (KIR2DL2/3) and HLA-C1 ligands are the most common KIR allele and HLA among the Caucasian population [167]. The cumulative number of Caucasians with HLA-C allotypes with asparagine at position 80 corresponding to CD158b approximates a third in this ethnic group [167]. Therefore, it is likely to have a donor with licensed and potentially active NK cell that express CD158b and could explain these findings. However, it is unclear how NK cell education preferentially expands the CD158b-expressing subpopulation. The present findings also raise the question if selection of suitable NK-cell donors which is based primarily on donor KIR genotype and recipient HLA genotype should be expanded to determine the fraction of active and hypoactive NK-cell populations according to the HLA and KIR genotype of the donor. These data warrant further validation with cells isolated from additional donors in ex vivo and animal model studies.

Previous studies of neuroblastoma cell death in culture compensated for uninduced levels of cell death by removing neuroblastoma cells from culture plates by normalizing assay results. Although this is an effective approach, the nine neuroblastoma cell lines that were tested had very different intrinsic sensitivities to flow cytometrybased cytotoxicity assays. Therefore, a new assay was developed that minimized basal levels of cell death. A large series of ADCC assays was conducted with this assay, demonstrating its feasibility. Unlike flow cytometry-based assays that have a very high sensitivity in detecting membrane disintegrity with viability staining, the ADCC assay required the tumor cells to have undergone cell death to quantify specific lysis. Therefore, this assay measures ADCC more conservatively than do flow cytometry- 
based methods, which may be advantageous for cell lines that can recover from an NKcell assault.

Importantly, NK cells were isolated from the peripheral blood of healthy deidentified allogeneic blood donors to assess ADCC by resting and IL-2-activated NK cells. Because of donor de-identification, each experiment was conducted with fresh cells from a different donor. Because the level of NK cell-mediated cytotoxicity may vary among individuals, comparing ADCC within an experiment with $\mathrm{NK}$ cells from one donor rather than between experiments with NK cells from different donors may lead to more biologically meaningful conclusions.

GD2 expression remained stable after ATRA-induced differentiation, which was expected because GD2 is a marker found in mature neurons [168]. Susceptibility to ADCC was improved after ATRA treatment. This is clinically relevant because patients receive anti-GD2 antibody therapy combined with ATRA [169]. Although not studied here, ATRA can induce higher expression of NKG2D ligands [170]. NKG2D serves as global co-stimulatory signal for various ITAM-associated pathways in NK cells, including CD16 signaling [171]. Although this could have been a plausible explanation as to why ATRA-treated tumors are highly susceptible to ADCC by anti-GD2 antibody alone without the need of IL-2 activation of NK cells, RNA sequencing did not reveal differential expression of the respective genes. Therefore, at this point, the mechanisms behind this are unclear, and future molecular studies are needed to explain the observed differences. In concordance with prior reports [172], the morphology of ATRAdifferentiated neuroblastoma cells varied greatly and could be due to differential expression of ATRA effector genes. For example, $R A R B$ and $C R A B P 2$ are upregulated in good differentiator cells and function as regulatory binding proteins that induce the transcription of ATRA target genes [173]. The transcriptional effects of ATRA also depend on its cellular concentration. Enzymes belonging to the CYP26 family are mainly responsible for ATRA homeostasis and were upregulated as well. If these genes can be used to predict differentiation efficiency warrants further studies.

Here, the feasibility of conducting tissue-matched ADCC experiments with patient NK cells and O-PDXs was demonstrated. Characterizing effector and tumor cells and conducting ADCC assays for individual patients in the laboratory may provide a valuable opportunity to identify and validate predictive biomarkers for therapy success and to study the potential vulnerabilities of tumor cells.

In this work, I found that IL-15, integrated in the current immunotherapy regimen for neuroblastoma, had significant preclinical anti-tumor activity in vitro and in vivo that exceeded the cytotoxic activity of IL-2. Curiously, the immunologic effects of IL-2 and IL-15 are overlapping because, despite the lack of sequence homology, both cytokine receptors share the same $\beta$ - and $\gamma$-chains with cytokine-specific $\alpha$-subunits [146]. Consistent with previous reports that have established the promoting role of IL-15 in NK cell maturation and survival [174], I found mice that completed therapy on the IL-15/IL$15 \mathrm{R} \alpha$ arm to have less immature tumor-infiltrating $\mathrm{CD} 27^{+} \mathrm{CD} 11 \mathrm{~b}^{-} \mathrm{NK}$ cells than animals in other groups. This suggests that IL-15 induces maturation in vivo, leading to higher 
proportions of mature NK cells with cytolytic capacity. Whether this finding is the cause for improved tumor growth suppression in vivo remains to be determined.

The safe use of recombinant human IL-15 was confirmed in a human phase I trial [141] in which adult patients with refractory solid tumors received daily bolus infusions for 12 consecutive days. Two patients cleared their lung lesions, indicative of partial activity. Detailed biologic profiling of the peripheral blood showed unique cellular kinetics in the treated patient that were characterized by an efflux in NK and memory $\mathrm{CD}^{+} \mathrm{T}$ cells from the circulation within 10 minutes of infusion start, followed by significant hyperproliferation, and hypoproliferation to restore homeostasis when IL-15 administration was ceased. Although recombinant human IL-15 is a promising agent, precomplexing IL-15 with IL-15R $\alpha$ has shown a 50-fold higher biologic activity $[175,176]$. ALT-803 is such a novel agent where the IL-15/IL-15R $\alpha$ complex was further fused to an IgG1 $F_{c}$ and a mutation in IL-15 (N72D) was introduced. These changes lead to longer half-life and enhanced biological activity of the recombinant cytokine. [177]. In a phase I studies, ALT-803 was administered subcutaneously once per week and tolerated with minimal side effects $[138,178]$. Based on my presented results, I propose IL-15 as an IL2 substitute in the current immunotherapy regimen for neuroblastoma. Therefore, I suggest the conduction of a larger preclinical trial to validate the results. Alternatively, the development of a clinical study could test the tolerability of recombinant human IL15 or ALT-803 with immunotherapy in children with relapsed/refractory neuroblastoma. The tumor response could be reported as a secondary endpoint and detailed correlative studies may further inform us regarding the biologic effect of IL-15 when given in combination with immunotherapy.

\section{Objective 2}

In my dissertation research, I developed and thoroughly characterized a humanized MISTRG neuroblastoma model for preclinical testing applications. I found that human NK cells in neuroblastoma-bearing mice have intact cytotoxic function in vivo. However, ex vivo cytolysis was attenuated in these animals. Molecular analysis revealed overlapping transcriptomic signatures between MISTRG-derived and patient NK cells that entailed genes involved in fundamental processes of NK cell function. Nevertheless, I discovered that the larger proportion of CD56 $6^{\text {bright }} \mathrm{NK}$ cells in mice constituted a major difference compared to patients. Thus, I suspect that impaired ex vivo cytotoxicity of MISTRG-derived NK cells could be related to higher proportions of CD56 ${ }^{\text {bright }} \mathrm{NK}$ cells, suggestive of immaturity of the NK cell compartment. Aside from mechanistic studies in these animals, I propose future testing of cytokines to therapeutically overcome the hypoactivity of NK cells.

Despite newly emerging humanized mouse models that foster human hematopoiesis, MISTRG mice are one of the only strains that permit endogenous reconstitution of macrophages/monocytes and NK cells because of a unique human cytokine milieu [115]. This is a critical feature of models used to observe the subtle intervention-mediated biologic changes without confounding the results with exogenous 
manipulations to maintain human engraftment. The biomedical application of MISTRG mice has been limited in the past because of the development of fatal anemia. However, modification of the transplantation protocol, as performed in this study, yields extended life spans and engrafted human tumors and hematopoiesis for chemoimmunotherapy testing for at least 6 weeks. In addition, the molecular signature of NK MISTRG-derived NK cells after transplantation as adult versus pup was overlapping. Therefore, I think that this finding may enable broader application of the humanized MISTRG neuroblastoma model to current areas of immunotherapy research.

Generally, two primary experimental approaches are used to design immunotherapeutic efficacy studies in murine models. Treatment can be started when mice are inoculated with tumor cells but have not formed detectable tumors (i.e., an early tumor model approach). Alternatively, mice can be treated when macroscopic tumor growth is present, (i.e., an established tumor model approach). Unless tumor engraftment rates are $100 \%$, testing experimental interventions in established tumors provides certainty that responses must be due to tumor shrinkage rather than engraftment failure, minimizing false-positive results. Therefore, it was important for this study to commence therapy when the tumors were macroscopically visible. By administering a chemoimmunotherapy regimen that is closely aligned with the current therapy for neuroblastoma [179], superior antitumor immunity was noted in MISTRG mice with endogenous hematopoiesis than with adoptively transferred mature NK cells, suggestive of intact cytotoxicity of endogenous NK cells in this model. This was corroborated by the fact that transplanted mice had considerably higher numbers of tumor-infiltrating immune cells than with adoptive NK cell transfer. When exploring the potential reasons for attenuated cytotoxicity of adoptively transferred NK cells, I found that mouse serum notably decreases NK cell function in vitro. Because adoptive NK cells mature in humans, I speculate that xenotransplantation into a mouse environment has a more profound negative effect on these cells than it does on NK cells that mature in the murine host and may have adapted to mouse-intrinsic conditions. Because it has been common practice to adoptively transfer NK cells from patients or allogeneic healthy donors to target neuroblastoma in humanized mouse models [180,181], my observation is relevant for future translational studies and supports the use of transplantation models. Lastly, this observation can be reconciled with findings from the pediatric trial that tested adoptive NK cell transfer for AML and failed to show an event-free survival benefit with infused NK cells, despite clear evidence that killer-cell immunoglobulin-like receptors (KIR)HLA-mismatched transplants can induce long-term tumor control [66].

By contrasting the transcriptomic findings in NK cells derived from patients to those of neuroblastoma-bearing MISTRG mice, I observed a larger proportion of cells with the NK2 gene profile in the mice. Furthermore, no association between these molecular findings and the functional capacity of the NK cells from MISTRG mice was evident. This raises the question of whether the molecular and functional phenotype of MISTRG-derived NK cells is caused by defective NK cell development or by tumorrelated factors and warrants future investigation. 
My animal studies were performed with only one PDX lines that was obtained from a patient with high-risk neuroblastoma but is well characterized [182]. However future studies should employ multiple different PDXs to span the heterogeneous spectrum of neuroblastoma and confirm whether NK cell dysfunction is also triggered by other neuroblastoma subtypes in vivo. MISTRG mice were reconstituted with allogeneic hematopoietic progenitor cells. NK cells that differentiate from human $\mathrm{CD}_{3}{ }^{+}$cells in mice and express mature NK cell receptors can reject human tumor because allogeneic hematopoietic stem and progenitor cell donors may be KIR-HLA-mismatched to the tumor, which can trigger rejection-type immune responses [28, 31]. Receptor-mediated rejection was possible in my experiments because the PDX line I used is deficient in HLA; however, because the tumor responses were not different among the chemotherapy groups of transplanted mice and those who received NK cell injections, I conclude that KIR-HLA mismatch played a minor role in tumor growth suppression.

The use of effector cells from the same patient who donated the tumor constitutes an alternative way to engraft the mice with tumor-matched effector cells. Although peripheral blood mononuclear cells are the easiest way to obtain such cells, CD $34^{+}$cells are preferred because these cells can induce long-term hematopoiesis in MISTRG mice. This can be achieved by collecting $\mathrm{CD} 34^{+}$cells after mobilization of these patients, which is routinely performed during autologous bone marrow transplantation [91]. In the future, ex vivo generation of hematopoietic stem cells from renewable sources, such as tumor-matched, patient-derived induced pluripotent stem cells, may be possible. Until then, the use of allogeneic donors will remain the mainstay for humanized transplantation and tumor models of immunotherapy. Lastly, my study does not definitively establish whether the varying NK cell cytotoxicity in neuroblastoma-bearing MISTRG mice is due to tumor or intrinsic insufficiencies of intact engraftment. Therefore, future experiments are needed to assess ex vivo NK cell function in tumor-bearing and tumor-free mice reconstituted with the same donor.

\section{Objective 3}

\section{NK cells in patients with neuroblastoma}

By studying NK cells from patients with neuroblastoma, I discovered that the natural cytotoxicity of NK cells is compromised in these individuals and correlates with tumor response to chemoimmunotherapy. My finding is important because it links the cellular capacity of a key effector cell involved in ADCC to antibody-mediated tumor shrinkage in the clinic. The underlying mechanisms leading to NK cell dysfunction can be further explored in the humanized MISTRG neuroblastoma model. Aside from mechanistic studies in these animal, I propose the study of cytokines to therapeutically overcome the hypoactivity of NK cells.

NK cells are classically divided into two populations: (1) CD56 ${ }^{\mathrm{dim}} \mathrm{CD} 16^{+} \mathrm{NK}$ cells that predominate in the peripheral blood and are regarded as the main effector cells 
in natural cytotoxicity and ADCC $[2,3]$ and (2) $\mathrm{CD}^{\text {bright }} \mathrm{CD}^{\text {(16 }}{ }^{-} \mathrm{NK}$ cells, enriched in secondary lymphoid tissues, that produce and secrete cytokines and chemokines to orchestrate inflammatory responses $[2,4,5]$. Studies of the natural history of NK cells consistently report profound declines in the number of CD56 $6^{\text {bright }} \mathrm{NK}$ cells and cytotoxic NK cell function with age [183-185]. Although I found substantially higher percentages of CD56 ${ }^{\text {bright }} \mathrm{NK}$ cells in patients with neuroblastoma than in healthy controls,

presumably due to young age and consistent with previous reports, NK cell function was markedly impaired in these individuals, suggesting intrinsic differences between patient and healthy NK cells. I quantified the differential contribution of CD56 $6^{\text {bright }} \mathrm{NK}$ cells to cytotoxicity and confirmed that this population does not participate in target cell cytolysis. Because I detected a correlation between CD56 ${ }^{\text {bright }} \mathrm{NK}$ cells in peripheral blood and ex vivo cytotoxicity, I think that the amount of CD56 $6^{\text {bright }} \mathrm{NK}$ cells contributes to the attenuated cytotoxicity and ultimately clinical tumor responses in these patients and warrants further investigation.

By performing single-cell RNA-seq of peripheral blood NK cells from patients with neuroblastoma, I identified two NK cell clusters with transcriptomic signatures involved in cytotoxicity and chemotaxis. My findings were similar to those reported by Crinier et al. who also identified two subpopulations in peripheral blood and splenic NK cells from healthy adults and defined the NK1 cell cluster as human CD56 ${ }^{\text {dim }}$ and the NK2 cell cluster as human CD56 ${ }^{\text {bright }}$ NK cells [125]. As part of my investigation, I conducted functional analysis of the molecularly profiled NK cells and discovered a functional-molecular association. Specifically, improved cytotoxicity occurred when the NK1 cluster predominated, and decreased cytotoxicity occurred with larger proportions of cells with the NK2 gene signature. Therefore, I conclude that the heterogeneous cytotoxic capacity of NK cells is related to the proportions of molecularly defined CD56 $6^{\text {dim }}$ and CD56 $6^{\text {bright }} \mathrm{NK}$ cells, which was corroborated by my functional and phenotypic analyses in 19 patients with neuroblastoma and healthy controls. Because previous studies have inferred that $\mathrm{CD} 56^{\text {bright }} \mathrm{CD} 16^{-} \mathrm{NK}$ cells are developmental precursors to the terminally differentiated CD56 ${ }^{\mathrm{dim}} \mathrm{CD} 16^{+}$subpopulation [8], I hypothesize that the increased proportion of CD56 $6^{\text {bright }} \mathrm{NK}$ cells in patients reflects immaturity of the NK cell compartment. Therefore, I propose future translational studies to assess whether cytokines, such as IL-15 that has established positive effects on NK cell development [136-138], can mitigate NK cell dysfunction in neuroblastoma in vivo. Because antibodies to CD56 are widely available and CD56 expression can be easily quantified by flow cytometry, confirmation of the prognostic importance of this biomarker may have substantial clinical implications.

By longitudinally phenotyping patients with neuroblastoma during chemoimmunotherapy, I found that these individuals had profound NK cell deficiency immediately after cytotoxic chemotherapy, but the cell count recovered to pre-treatment level before the next cycle of chemotherapy. NK cytopenia was less severe with cisplatin and etoposide, when compared with cyclophosphamide/topotecan and cyclophosphamide/doxorubicin/vincristine. This observation highlights the importance of choosing chemotherapeutic agents that are known to be effective against the primary 
cancer yet are also associated with less severe lymphotoxicity for chemoimmunotherapy trials [186].

After autologous transplantation, haploidentical NK cell infusion may partially correct the profound NK cytopenia. Notably, all patients who received more than 25 $\times 10^{6} / \mathrm{kg}$ NK cells had transient engraftment of donor cells. If donor cells are insufficient, ex vivo expansion may be a feasible option to increase the infused cell dose [187]. Other than donor factors, recipient factors might affect NK cell expansion after adoptive transfer. In this study, alloreactive NK cell expansion appeared to be higher in patients who had more residual disease, categorized as those with stable disease. Furthermore, all seven patients who did not have inhibitory HLA-Bw4 ligands against the donor CD158e1 (KIR3DL1) are survivors. This observation is line with previous findings that KIR3DL1 and HLA-B epitopes are key modulators of NK response to anti-GD2 antibody therapy in patients with neuroblastoma [159].

Together, these findings demonstrate that donor factors, NK cell product characteristics, patient factors, and tumor response may all contribute to the complex NK kinetics and dynamics in patients with neuroblastoma receiving chemoimmunotherapy. This study encourages further investigations regarding NK cell receptor biology and function in neuroblastoma antibody therapy.

\section{Adoptive NK cell transfer for AML therapy}

Hematopoietic cell transplantation from KIR-HLA-mismatched donors is associated with a marked event-free survival benefit in patients with AML, [65-67] but the benefit of NK cell infusion as a consolidation therapy remains unclear for children with AML. Therefore, the first pediatric phase II study was conducted to determine whether adoptively transferred NK cells from haploidentical and KIR-HLA mismatched donors improve event-free-survival in children with intermediate-risk AML who completed chemotherapy and were in first remission. Despite tolerability and transient engraftment of donor NK cells in most individuals, adoptive transfer of NK cells did not decrease relapse or increase survival compared to chemotherapy alone. The lack of survival benefit in this cohort may be explained due to limited persistence of donor NK cells and low donor NK cell infusion dose.

The bone marrow is the main site for NK cell production, and the average life span of circulating NK cells is approximately two weeks [6]. Consistent with that, most patients in this study had decreasing donor chimerism levels after two weeks from the time of NK cell infusion, suggesting that the kinetics of adoptively transferred NK cells follow the same pattern of decay as that of primary NK cells. [6] During hematopoietic transplantation, donor progenitor cells are a permanent source for alloreactive NK cells allowing for continued tumor surveillance, whereas adoptively transferred NK cells represent a limited cell pool. Therefore, it can be argued that the therapeutic window for alloreactive NK cells had long passed by the time patients in this study experienced relapse, which occurred from six to 21 months after NK cell infusion. 
The average peak and week 4 donor NK cell chimerisms in this study were significantly lower compared to reports from a previous phase I trial that used an identical lymphodepleting chemotherapy regimen [69]. It is believed that this discrepancy could be due to the low median number of infused NK cells $\left(12.5\right.$ vs. $29 \times 10^{6} \mathrm{NK}$ cells $/ \mathrm{kg}$ ). Although there was not a linear correlation between peak donor NK cell chimerism and infused NK cell dose, a causal relationship may still exist. The peripheral blood donor chimerism has been shown not to sensitively reflect bone marrow status [188] and thus may not fully quantitate residual donor NK cells, explaining the lack of correlation. Because the two-step process to isolate NK cells was identical in both studies, and because the proportion of young patients with low body weight was similar as well, it is possible that the low NK cell dose was due to donor-related factors. Whether extending the time of IL-2 administration or alternatively administering IL-15 may lead to further expansion and donor NK cell persistence in vivo remains to be determined in future trials. Regardless, decreased numbers of adoptively transferred NK cells may have affected tumor clearance, thereby enabling the re-emergence of leukemic cell clones in patients with relapse. To support this, six of eight patients with disease relapse received less than the median NK cell dose. Of the 10 patients from the phase I trial, 6 had intermediate-risk AML and have remained relapse-free after a follow-up of approximately 32 months. Conversely, this cohort was infused with a much higher median NK cell number $\left(29 \times 10^{6} \mathrm{NK}\right.$ cells $\left./ \mathrm{kg}\right)$ and contained only one patient (UPN 7) who received less than the median phase II NK cell dose.

Children in this study received purified but otherwise unmanipulated NK cells. It is conceivable that activated and expanded or chimeric antigen receptor (CAR)expressing NK cells may have more applicability in the future. Although NK cells are more difficult to genetically modify for this purpose than $\mathrm{T}$ cells, they have a limited life span in vivo and potentially a more favorable toxicity profile than the latter. Alternatively, adoptively transferred NK cells could be combined with a monoclonal antibody specific to AML blasts, such as CD33, to augment ADCC. Due to the transient persistence of donor NK cells and monoclonal antibody, long-term myelotoxicity with this therapy is theoretically less likely to occur than with CAR-T cells [189]. Together, the therapeutic usefulness and clinical applications of ex vivo modified NK cells is promising but remains to be determined. Although the number of KIR-HLA mismatched NK cells was not associated with survival, certain cell surface markers were and could be used as biomarker to predict individual responses to NK cell therapy in the future. However, further validation of these markers in clinical trials is needed. 


\section{LIST OF REFERENCES}

1. Lanier LL (1989) Identity of Leu-19 (CD56) leukocyte differentiation antigen and neural cell adhesion molecule. J Exp Med 169:2233-2238.

https://doi.org/10.1084/jem.169.6.2233

2. Fehniger TA, Cooper MA, Nuovo GJ, et al (2003) CD56bright natural killer cells are present in human lymph nodes and are activated by T cell-derived IL-2: a potential new link between adaptive and innate immunity. Blood 101:3052-3057. https://doi.org/10.1182/blood-2002-09-2876

3. Perussia B (1998) Fe receptors on natural killer cells. Curr Top Microbiol Immunol 230:63-88

4. Cooper MA (2001) Human natural killer cells: a unique innate immunoregulatory role for the CD56bright subset. Blood 97:3146-3151.

https://doi.org/10.1182/blood.V97.10.3146

5. Nagler A, Lanier LL, Cwirla S, Phillips JH (1989) Comparative studies of human FcRIII-positive and negative natural killer cells. J Immunol Baltim Md 1950 143:3183-3191

6. Zhang Y, Wallace DL, de Lara CM, et al (2007) In vivo kinetics of human natural killer cells: the effects of ageing and acute and chronic viral infection. Immunology 121:258-265. https://doi.org/10.1111/j.1365-2567.2007.02573.x

7. Macallan DC, Wallace DL, Irvine AJ, et al (2003) Rapid turnover of T cells in acute infectious mononucleosis. Eur J Immunol 33:2655-2665.

https://doi.org/10.1002/eji.200324295

8. Lanier LL, Le AM, Civin CI, et al (1986) The relationship of CD16 (Leu-11) and Leu-19 (NKH-1) antigen expression on human peripheral blood NK cells and cytotoxic T lymphocytes. J Immunol Baltim Md 1950 136:4480-4486

9. Freud AG, Becknell B, Roychowdhury S, et al (2005) A human CD34(+) subset resides in lymph nodes and differentiates into CD56bright natural killer cells. Immunity 22:295-304. https://doi.org/10.1016/j.immuni.2005.01.013

10. Romagnani C, Juelke K, Falco M, et al (2007) CD56brightCD16- Killer Ig-Like Receptor- NK Cells Display Longer Telomeres and Acquire Features of CD56dim NK Cells upon Activation. J Immunol 178:4947-4955. https://doi.org/10.4049/jimmunol.178.8.4947

11. Wu C, Li B, Lu R, et al (2014) Clonal Tracking of Rhesus Macaque Hematopoiesis Highlights a Distinct Lineage Origin for Natural Killer Cells. Cell Stem Cell 14:486-499. https://doi.org/10.1016/j.stem.2014.01.020 
12. Trotta R (2005) Differential expression of SHIP1 in CD56bright and CD56dim NK cells provides a molecular basis for distinct functional responses to monokine costimulation. Blood 105:3011-3018. https://doi.org/10.1182/blood-2004-10-4072

13. Lanier LL (1998) NK cell receptors. Annu Rev Immunol 16:359-393. https://doi.org/10.1146/annurev.immunol.16.1.359

14. Orange JS (2013) Natural killer cell deficiency. J Allergy Clin Immunol 132:515525. https://doi.org/10.1016/j.jaci.2013.07.020

15. Biron CA, Byron KS, Sullivan JL (1989) Severe Herpesvirus Infections in an Adolescent without Natural Killer Cells. N Engl J Med 320:1731-1735. https://doi.org/10.1056/NEJM198906293202605

16. Fleisher G, Starr S, Koven N, et al (1982) A non-X-linked syndrome with susceptibility to severe epstein-barr virus infections. J Pediatr 100:727-730. https://doi.org/10.1016/S0022-3476(82)80572-6

17. Eidenschenk C, Dunne J, Jouanguy E, et al (2006) A Novel Primary Immunodeficiency with Specific Natural-Killer Cell Deficiency Maps to the Centromeric Region of Chromosome 8. Am J Hum Genet 78:721-727. https://doi.org/10.1086/503269

18. Hanna S, Béziat V, Jouanguy E, et al (2015) A homozygous mutation of RTEL1 in a child presenting with an apparently isolated natural killer cell deficiency. $\mathrm{J}$ Allergy Clin Immunol 136:1113-1114. https://doi.org/10.1016/j.jaci.2015.04.021

19. Mace EM, Hsu AP, Monaco-Shawver L, et al (2013) Mutations in GATA2 cause human NK cell deficiency with specific loss of the CD56bright subset. Blood 121:2669-2677. https://doi.org/10.1182/blood-2012-09-453969

20. Jawahar S, Moody C, Chan M, et al (2007) Natural killer (NK) cell deficiency associated with an epitope-deficient Fc receptor type IIIA (CD16-II). Clin Exp Immunol 103:408-413. https://doi.org/10.1111/j.1365-2249.1996.tb08295.x

21. de Vries E, Koene HR, Vossen JM, et al (1996) Identification of an unusual Fc gamma receptor IIIa (CD16) on natural killer cells in a patient with recurrent infections. Blood 88:3022-3027

22. Grier JT, Forbes LR, Monaco-Shawver L, et al (2012) Human immunodeficiencycausing mutation defines CD16 in spontaneous NK cell cytotoxicity. J Clin Invest 122:3769-3780. https://doi.org/10.1172/JCI64837

23. Spinner MA, Sanchez LA, Hsu AP, et al (2014) GATA2 deficiency: a protean disorder of hematopoiesis, lymphatics, and immunity. Blood 123:809-821. https://doi.org/10.1182/blood-2013-07-515528 
24. Imai K, Matsuyama S, Miyake S, et al (2000) Natural cytotoxic activity of peripheral-blood lymphocytes and cancer incidence: an 11-year follow-up study of a general population. Lancet Lond Engl 356:1795-1799. https://doi.org/10.1016/S0140-6736(00)03231-1

25. Murphy K (2014) Janeway's immunobiology. Garland Science, Place of publication not identified

26. Bryceson YT, March ME, Ljunggren H-G, Long EO (2006) Activation, coactivation, and costimulation of resting human natural killer cells. Immunol Rev 214:73-91. https://doi.org/10.1111/j.1600-065X.2006.00457.x

27. Yu J, Heller G, Chewning J, et al (2007) Hierarchy of the Human Natural Killer Cell Response Is Determined by Class and Quantity of Inhibitory Receptors for Self-HLA-B and HLA-C Ligands. J Immunol 179:5977-5989. https://doi.org/10.4049/jimmunol.179.9.5977

28. Ljunggren HG, Kärre K (1990) In search of the "missing self": MHC molecules and NK cell recognition. Immunol Today 11:237-244

29. Brodin P, Lakshmikanth T, Johansson S, et al (2009) The strength of inhibitory input during education quantitatively tunes the functional responsiveness of individual natural killer cells. Blood 113:2434-2441. https://doi.org/10.1182/blood2008-05-156836

30. Long EO (2008) Negative signaling by inhibitory receptors: the NK cell paradigm. Immunol Rev 224:70-84. https://doi.org/10.1111/j.1600-065X.2008.00660.x

31. Leung W, Handgretinger R, Iyengar R, et al (2007) Inhibitory KIR-HLA receptorligand mismatch in autologous haematopoietic stem cell transplantation for solid tumour and lymphoma. Br J Cancer 97:539-542. https://doi.org/10.1038/sj.bjc.6603913

32. Kim S, Poursine-Laurent J, Truscott SM, et al (2005) Licensing of natural killer cells by host major histocompatibility complex class I molecules. Nature 436:709713. https://doi.org/10.1038/nature03847

33. Bélanger S, Tu MM, Rahim MMA, et al (2012) Impaired natural killer cell selfeducation and "missing-self" responses in Ly49-deficient mice. Blood 120:592602. https://doi.org/10.1182/blood-2012-02-408732

34. Höglund P, Brodin P (2010) Current perspectives of natural killer cell education by MHC class I molecules. Nat Rev Immunol 10:724-734. https://doi.org/10.1038/nri2835

35. Cudkowicz G, Bennett M (1971) Peculiar immunobiology of bone marrow allografts. II. Rejection of parental grafts by resistant F 1 hybrid mice. J Exp Med 134:1513-1528 
36. Murphy WJ, Kumar V, Bennett M (1987) Rejection of bone marrow allografts by mice with severe combined immune deficiency (SCID). Evidence that natural killer cells can mediate the specificity of marrow graft rejection. J Exp Med 165:12121217

37. Furukawa H, Yabe T, Watanabe K, et al (1999) Tolerance of NK and LAK activity for HLA class I-deficient targets in a TAP1-deficient patient (bare lymphocyte syndrome type I). Hum Immunol 60:32-40

38. Liao NS, Bix M, Zijlstra M, et al (1991) MHC class I deficiency: susceptibility to natural killer (NK) cells and impaired NK activity. Science 253:199-202

39. Höglund P, Ohlén C, Carbone E, et al (1991) Recognition of beta 2-microglobulinnegative (beta $2 \mathrm{~m}$-) T-cell blasts by natural killer cells from normal but not from beta $2 \mathrm{~m}$ - mice: nonresponsiveness controlled by beta $2 \mathrm{~m}$ - bone marrow in chimeric mice. Proc Natl Acad Sci U S A 88:10332-10336

40. Ljunggren HG, Van Kaer L, Ploegh HL, Tonegawa S (1994) Altered natural killer cell repertoire in Tap-1 mutant mice. Proc Natl Acad Sci U S A 91:6520-6524

41. Zimmer J, Donato L, Hanau D, et al (1998) Activity and phenotype of natural killer cells in peptide transporter (TAP)-deficient patients (type I bare lymphocyte syndrome). J Exp Med 187:117-122

42. Bauer $\mathrm{S}$, Groh V, Wu J, et al (1999) Activation of NK cells and T cells by NKG2D, a receptor for stress-inducible MICA. Science 285:727-729

43. Cerwenka A, Lanier LL (2003) NKG2D ligands: unconventional MHC class I-like molecules exploited by viruses and cancer. Tissue Antigens 61:335-343

44. Cosman D, Müllberg J, Sutherland CL, et al (2001) ULBPs, novel MHC class Irelated molecules, bind to CMV glycoprotein UL16 and stimulate NK cytotoxicity through the NKG2D receptor. Immunity 14:123-133

45. Cheng NC, Beitsma M, Chan A, et al (1996) Lack of class I HLA expression in neuroblastoma is associated with high $\mathrm{N}$-myc expression and hypomethylation due to loss of the MEMO-1 locus. Oncogene 13:1737-1744

46. Zingoni A, Fionda C, Borrelli C, et al (2017) Natural Killer Cell Response to Chemotherapy-Stressed Cancer Cells: Role in Tumor Immunosurveillance. Front Immunol 8:1194. https://doi.org/10.3389/fimmu.2017.01194

47. Zhou F (2009) Molecular Mechanisms of IFN- $\gamma$ to Up-Regulate MHC Class I Antigen Processing and Presentation. Int Rev Immunol 28:239-260. https://doi.org/10.1080/08830180902978120 
48. Zent CS, Chen JB, Kurten RC, et al (2004) Alemtuzumab (CAMPATH 1H) does not kill chronic lymphocytic leukemia cells in serum free medium. Leuk Res 28:495-507. https://doi.org/10.1016/j.leukres.2003.09.011

49. Brentjens RJ, Davila ML, Riviere I, et al (2013) CD19-Targeted T Cells Rapidly Induce Molecular Remissions in Adults with Chemotherapy-Refractory Acute Lymphoblastic Leukemia. Sci Transl Med 5:177ra38-177ra38. https://doi.org/10.1126/scitranslmed.3005930

50. Larkin J, Chiarion-Sileni V, Gonzalez R, et al (2015) Combined Nivolumab and Ipilimumab or Monotherapy in Untreated Melanoma. N Engl J Med 373:23-34. https://doi.org/10.1056/NEJMoa1504030

51. Slamon DJ, Leyland-Jones B, Shak S, et al (2001) Use of Chemotherapy plus a Monoclonal Antibody against HER2 for Metastatic Breast Cancer That Overexpresses HER2. N Engl J Med 344:783-792. https://doi.org/10.1056/NEJM200103153441101

52. Hurwitz H, Fehrenbacher L, Novotny W, et al (2004) Bevacizumab plus Irinotecan, Fluorouracil, and Leucovorin for Metastatic Colorectal Cancer. N Engl J Med 350:2335-2342. https://doi.org/10.1056/NEJMoa032691

53. Wellenstein MD, de Visser KE (2018) Cancer-Cell-Intrinsic Mechanisms Shaping the Tumor Immune Landscape. Immunity 48:399-416. https://doi.org/10.1016/j.immuni.2018.03.004

54. Groth A, Klöss S, Pogge von Strandmann E, et al (2011) Mechanisms of Tumor and Viral Immune Escape from Natural Killer Cell-Mediated Surveillance. J Innate Immun 3:344-354. https://doi.org/10.1159/000327014

55. Groh V, Wu J, Yee C, Spies T (2002) Tumour-derived soluble MIC ligands impair expression of NKG2D and T-cell activation. Nature 419:734-738. https://doi.org/10.1038/nature01112

56. Aldeghaither DS, Zahavi DJ, Murray JC, et al (2018) A Mechanism of Resistance to Antibody-Targeted Immune Attack. Cancer Immunol Res. https://doi.org/10.1158/2326-6066.CIR-18-0266

57. Gao J, Zheng Q, Xin N, et al (2017) CD155, an onco-immunologic molecule in human tumors. Cancer Sci 108:1934-1938. https://doi.org/10.1111/cas.13324

58. Zhang Q, Bi J, Zheng X, et al (2018) Blockade of the checkpoint receptor TIGIT prevents NK cell exhaustion and elicits potent anti-tumor immunity. Nat Immunol 19:723-732. https://doi.org/10.1038/s41590-018-0132-0

59. Stanietsky N, Simic H, Arapovic J, et al (2009) The interaction of TIGIT with PVR and PVRL2 inhibits human NK cell cytotoxicity. Proc Natl Acad Sci 106:1785817863. https://doi.org/10.1073/pnas.0903474106 
60. Carlsten M, Norell H, Bryceson YT, et al (2009) Primary human tumor cells expressing CD155 impair tumor targeting by down-regulating DNAM-1 on NK cells. J Immunol Baltim Md 1950 183:4921-4930.

https://doi.org/10.4049/jimmunol.0901226

61. Bottino C, Castriconi R, Pende D, et al (2003) Identification of PVR (CD155) and Nectin-2 (CD112) as cell surface ligands for the human DNAM-1 (CD226) activating molecule. J Exp Med 198:557-567. https://doi.org/10.1084/jem.20030788

62. Li M, Xia P, Du Y, et al (2014) T-cell immunoglobulin and ITIM domain (TIGIT) receptor/poliovirus receptor (PVR) ligand engagement suppresses interferon- $\gamma$ production of natural killer cells via $\beta$-arrestin 2-mediated negative signaling. J Biol Chem 289:17647-17657. https://doi.org/10.1074/jbc.M114.572420

63. Pistoia V, Morandi F, Bianchi G, et al (2013) Immunosuppressive Microenvironment in Neuroblastoma. Front Oncol 3:. https://doi.org/10.3389/fonc.2013.00167

64. Gabrilovich DI, Ostrand-Rosenberg S, Bronte V (2012) Coordinated regulation of myeloid cells by tumours. Nat Rev Immunol 12:253-268.

https://doi.org/10.1038/nri3175

65. Ruggeri L, Mancusi A, Capanni M, et al (2007) Donor natural killer cell allorecognition of missing self in haploidentical hematopoietic transplantation for acute myeloid leukemia: challenging its predictive value. Blood 110:433-440. https://doi.org/10.1182/blood-2006-07-038687

66. Ruggeri L (2002) Effectiveness of Donor Natural Killer Cell Alloreactivity in Mismatched Hematopoietic Transplants. Science 295:2097-2100. https://doi.org/10.1126/science. 1068440

67. Pende D, Marcenaro S, Falco M, et al (2009) Anti-leukemia activity of alloreactive NK cells in KIR ligand-mismatched haploidentical HSCT for pediatric patients: evaluation of the functional role of activating KIR and redefinition of inhibitory KIR specificity. Blood 113:3119-3129. https://doi.org/10.1182/blood-2008-06164103

68. Miller JS, Soignier Y, Panoskaltsis-Mortari A, et al (2005) Successful adoptive transfer and in vivo expansion of human haploidentical NK cells in patients with cancer. Blood 105:3051-3057. https://doi.org/10.1182/blood-2004-07-2974

69. Rubnitz JE, Inaba H, Ribeiro RC, et al (2010) NKAML: A Pilot Study to Determine the Safety and Feasibility of Haploidentical Natural Killer Cell Transplantation in Childhood Acute Myeloid Leukemia. J Clin Oncol 28:955-959. https://doi.org/10.1200/JCO.2009.24.4590 
70. Curti A, Ruggeri L, D'Addio A, et al (2011) Successful transfer of alloreactive haploidentical KIR ligand-mismatched natural killer cells after infusion in elderly high risk acute myeloid leukemia patients. Blood 118:3273-3279. https://doi.org/10.1182/blood-2011-01-329508

71. Rubnitz JE, Inaba H, Dahl G, et al (2010) Minimal residual disease-directed therapy for childhood acute myeloid leukaemia: results of the AML02 multicentre trial. Lancet Oncol 11:543-552. https://doi.org/10.1016/S1470-2045(10)70090-5

72. Gamis AS, Alonzo TA, Meshinchi S, et al (2014) Gemtuzumab ozogamicin in children and adolescents with de novo acute myeloid leukemia improves event-free survival by reducing relapse risk: results from the randomized phase III Children's Oncology Group trial AAML0531. J Clin Oncol Off J Am Soc Clin Oncol 32:3021-3032. https://doi.org/10.1200/JCO.2014.55.3628

73. Tonn T, Schwabe D, Klingemann HG, et al (2013) Treatment of patients with advanced cancer with the natural killer cell line NK-92. Cytotherapy 15:1563-1570. https://doi.org/10.1016/j.jcyt.2013.06.017

74. Bachanova V, Cooley S, Defor TE, et al (2014) Clearance of acute myeloid leukemia by haploidentical natural killer cells is improved using IL-2 diphtheria toxin fusion protein. Blood 123:3855-3863. https://doi.org/10.1182/blood-2013-10532531

75. Szmania S, Lapteva N, Garg T, et al (2015) Ex Vivo-expanded Natural Killer Cells Demonstrate Robust Proliferation In Vivo in High-risk Relapsed Multiple Myeloma Patients: J Immunother 38:24-36. https://doi.org/10.1097/CJI.0000000000000059

76. Rubnitz JE, Inaba H, Kang G, et al (2015) Natural killer cell therapy in children with relapsed leukemia: NK Cell Therapy in Pediatric Leukemia. Pediatr Blood Cancer 62:1468-1472. https://doi.org/10.1002/pbc.25555

77. Shaffer BC, Le Luduec J-B, Forlenza C, et al (2016) Phase II Study of Haploidentical Natural Killer Cell Infusion for Treatment of Relapsed or Persistent Myeloid Malignancies Following Allogeneic Hematopoietic Cell Transplantation. Biol Blood Marrow Transplant 22:705-709. https://doi.org/10.1016/j.bbmt.2015.12.028

78. Yang Y, Lim O, Kim TM, et al (2016) Phase I Study of Random Healthy DonorDerived Allogeneic Natural Killer Cell Therapy in Patients with Malignant Lymphoma or Advanced Solid Tumors. Cancer Immunol Res 4:215-224. https://doi.org/10.1158/2326-6066.CIR-15-0118

79. Dolstra H, Roeven MWH, Spanholtz J, et al (2017) Successful Transfer of Umbilical Cord Blood CD34+ Hematopoietic Stem and Progenitor-derived NK Cells in Older Acute Myeloid Leukemia Patients. Clin Cancer Res Off J Am Assoc Cancer Res 23:4107-4118. https://doi.org/10.1158/1078-0432.CCR-16-2981 
80. Yu AL, Gilman AL, Ozkaynak MF, et al (2010) Anti-GD2 Antibody with GMCSF, Interleukin-2, and Isotretinoin for Neuroblastoma. N Engl J Med 363:13241334. https://doi.org/10.1056/NEJMoa0911123

81. Schulz G, Cheresh DA, Varki NM, et al (1984) Detection of ganglioside GD2 in tumor tissues and sera of neuroblastoma patients. Cancer Res 44:5914-5920

82. Sidell N (1982) Retinoic acid-induced growth inhibition and morphologic differentiation of human neuroblastoma cells in vitro. J Natl Cancer Inst 68:589596

83. Henney CS, Kuribayashi K, Kern DE, Gillis S (1981) Interleukin-2 augments natural killer cell activity. Nature 291:335-338. https://doi.org/10.1038/291335a0

84. Kushner BH, Cheung NK (1989) GM-CSF enhances 3F8 monoclonal antibodydependent cellular cytotoxicity against human melanoma and neuroblastoma. Blood 73:1936-1941

85. Fukuda M, Horibe K, Furukawa K (1998) Enhancement of in vitro and in vivo antitumor activity of anti-GD2 monoclonal antibody 220-51 against human neuroblastoma by granulocyte-macrophage colony-stimulating factor and granulocyte colony-stimulating factor. Int J Mol Med 2:471-475

86. SEER Cancer Statistics Review. National Cancer Institute, Bethesa, MD

87. Nickerson HJ, Matthay KK, Seeger RC, et al (2000) Favorable biology and outcome of stage IV-S neuroblastoma with supportive care or minimal therapy: a Children's Cancer Group study. J Clin Oncol Off J Am Soc Clin Oncol 18:477486. https://doi.org/10.1200/JCO.2000.18.3.477

88. London WB, Castleberry RP, Matthay KK, et al (2005) Evidence for an age cutoff greater than 365 days for neuroblastoma risk group stratification in the Children's Oncology Group. J Clin Oncol Off J Am Soc Clin Oncol 23:6459-6465. https://doi.org/10.1200/JCO.2005.05.571

89. Thompson D, Vo KT, London WB, et al (2016) Identification of patient subgroups with markedly disparate rates of MYCN amplification in neuroblastoma: A report from the International Neuroblastoma Risk Group project: Predictors of MNA in Neuroblastoma. Cancer 122:935-945. https://doi.org/10.1002/cncr.29848

90. Matthay KK, Villablanca JG, Seeger RC, et al (1999) Treatment of high-risk neuroblastoma with intensive chemotherapy, radiotherapy, autologous bone marrow transplantation, and 13-cis-retinoic acid. Children's Cancer Group. N Engl J Med 341:1165-1173. https://doi.org/10.1056/NEJM199910143411601

91. Park JR, Kreissman SG, London WB, et al (2016) A phase III randomized clinical trial (RCT) of tandem myeloablative autologous stem cell transplant (ASCT) using peripheral blood stem cell (PBSC) as consolidation therapy for high-risk 
neuroblastoma (HR-NB): A Children's Oncology Group (COG) study. J Clin Oncol 34:

92. Navid F, Sondel PM, Barfield R, et al (2014) Phase I trial of a novel anti-GD2 monoclonal antibody, Hu14.18K322A, designed to decrease toxicity in children with refractory or recurrent neuroblastoma. J Clin Oncol Off J Am Soc Clin Oncol 32:1445-1452. https://doi.org/10.1200/JCO.2013.50.4423

93. Talleur AC, Triplett BM, Federico S, et al (2017) Consolidation Therapy for Newly Diagnosed Pediatric Patients with High-Risk Neuroblastoma Using Busulfan/Melphalan, Autologous Hematopoietic Cell Transplantation, Anti-GD2 Antibody, Granulocyte-Macrophage Colony-Stimulating Factor, Interleukin-2, and Haploidentical Natural Killer Cells. Biol Blood Marrow Transplant J Am Soc Blood Marrow Transplant 23:1910-1917.

https://doi.org/10.1016/j.bbmt.2017.07.011

94. Fox BA, Schendel DJ, Butterfield LH, et al (2011) Defining the critical hurdles in cancer immunotherapy. J Transl Med 9:. https://doi.org/10.1186/1479-5876-9-214

95. Gengenbacher N, Singhal M, Augustin HG (2017) Preclinical mouse solid tumour models: status quo, challenges and perspectives. Nat Rev Cancer 17:751-765. https://doi.org/10.1038/nrc.2017.92

96. Sena ES, van der Worp HB, Bath PMW, et al (2010) Publication bias in reports of animal stroke studies leads to major overstatement of efficacy. PLoS Biol 8:e1000344. https://doi.org/10.1371/journal.pbio.1000344

97. Perel P, Roberts I, Sena E, et al (2007) Comparison of treatment effects between animal experiments and clinical trials: systematic review. BMJ 334:197. https://doi.org/10.1136/bmj.39048.407928.BE

98. Woosley RL, Cossman J (2007) Drug Development and the FDA's Critical Path Initiative. Clin Pharmacol Ther 81:129-133. https://doi.org/10.1038/sj.clpt.6100014

99. Linette GP, Stadtmauer EA, Maus MV, et al (2013) Cardiovascular toxicity and titin cross-reactivity of affinity-enhanced T cells in myeloma and melanoma. Blood 122:863-871. https://doi.org/10.1182/blood-2013-03-490565

100. Cameron BJ, Gerry AB, Dukes J, et al (2013) Identification of a Titin-derived HLA-A1-presented peptide as a cross-reactive target for engineered MAGE A3directed T cells. Sci Transl Med 5:197ra103. https://doi.org/10.1126/scitranslmed.3006034

101. Raman MCC, Rizkallah PJ, Simmons R, et al (2016) Direct molecular mimicry enables off-target cardiovascular toxicity by an enhanced affinity TCR designed for cancer immunotherapy. Sci Rep 6:. https://doi.org/10.1038/srep18851 
102. Barten R, Trowsdale J (1999) The human Ly-49L gene. Immunogenetics 49:731734. https://doi.org/10.1007/s002510050675

103. Stewart E, Federico SM, Chen X, et al (2017) Orthotopic patient-derived xenografts of paediatric solid tumours. Nature 549:96-100.

https://doi.org/10.1038/nature23647

104. Stewart E, McEvoy J, Wang H, et al (2018) Identification of Therapeutic Targets in Rhabdomyosarcoma through Integrated Genomic, Epigenomic, and Proteomic Analyses. Cancer Cell 34:411-426.e19. https://doi.org/10.1016/j.ccell.2018.07.012

105. Lapidot T, Pflumio F, Doedens M, et al (1992) Cytokine stimulation of multilineage hematopoiesis from immature human cells engrafted in SCID mice. Science 255:1137-1141. https://doi.org/10.1126/science.1372131

106. McCune J, Namikawa R, Kaneshima H, et al (1988) The SCID-hu mouse: murine model for the analysis of human hematolymphoid differentiation and function. Science 241:1632-1639. https://doi.org/10.1126/science.2971269

107. Mosier DE, Gulizia RJ, Baird SM, Wilson DB (1988) Transfer of a functional human immune system to mice with severe combined immunodeficiency. Nature 335:256-259. https://doi.org/10.1038/335256a0

108. Ito M (2002) NOD/SCID/gamma cnull mouse: an excellent recipient mouse model for engraftment of human cells. Blood 100:3175-3182. https://doi.org/10.1182/blood-2001-12-0207

109. Traggiai E (2004) Development of a Human Adaptive Immune System in Cord Blood Cell-Transplanted Mice. Science 304:104-107. https://doi.org/10.1126/science.1093933

110. Holyoake T (1999) Functional differences between transplantable human hematopoietic stem cells from fetal liver, cord blood, and adult marrow. Exp Hematol 27:1418-1427. https://doi.org/10.1016/S0301-472X(99)00078-8

111. Shultz LD, Lyons BL, Burzenski LM, et al (2005) Human Lymphoid and Myeloid Cell Development in NOD/LtSz-scid IL2R null Mice Engrafted with Mobilized Human Hemopoietic Stem Cells. J Immunol 174:6477-6489. https://doi.org/10.4049/jimmunol.174.10.6477

112. Herndler-Brandstetter D, Shan L, Yao Y, et al (2017) Humanized mouse model supports development, function, and tissue residency of human natural killer cells. Proc Natl Acad Sci 114:E9626-E9634. https://doi.org/10.1073/pnas.1705301114

113. Wunderlich M, Chou F-S, Link KA, et al (2010) AML xenograft efficiency is significantly improved in NOD/SCID-IL2RG mice constitutively expressing human SCF, GM-CSF and IL-3. Leukemia 24:1785-1788. https://doi.org/10.1038/leu.2010.158 
114. Billerbeck E, Barry WT, Mu K, et al (2011) Development of human CD4+FoxP3+ regulatory $\mathrm{T}$ cells in human stem cell factor-, granulocyte-macrophage colonystimulating factor-, and interleukin-3-expressing NOD-SCID IL2R $\gamma($ null) humanized mice. Blood 117:3076-3086. https://doi.org/10.1182/blood-2010-08301507

115. Rongvaux A, Willinger T, Martinek J, et al (2014) Development and function of human innate immune cells in a humanized mouse model. Nat Biotechnol 32:364372. https://doi.org/10.1038/nbt.2858

116. De La Rochere P, Guil-Luna S, Decaudin D, et al (2018) Humanized Mice for the Study of Immuno-Oncology. Trends Immunol 39:748-763.

https://doi.org/10.1016/j.it.2018.07.001

117. Lan P (2006) Reconstitution of a functional human immune system in immunodeficient mice through combined human fetal thymus/liver and CD34+ cell transplantation. Blood 108:487-492. https://doi.org/10.1182/blood-2005-11-4388

118. Knorr DA, Ni Z, Hermanson D, et al (2013) Clinical-Scale Derivation of Natural Killer Cells From Human Pluripotent Stem Cells for Cancer Therapy. STEM CELLS Transl Med 2:274-283. https://doi.org/10.5966/sctm.2012-0084

119. Denman CJ, Senyukov VV, Somanchi SS, et al (2012) Membrane-Bound IL-21 Promotes Sustained Ex Vivo Proliferation of Human Natural Killer Cells. PLoS ONE 7:e30264. https://doi.org/10.1371/journal.pone.0030264

120. Chapman K, Pullen N, Coney L, et al (2009) Preclinical development of monoclonal antibodies: considerations for the use of non-human primates. mAbs $1: 505-516$

121. Kamath AV (2016) Translational pharmacokinetics and pharmacodynamics of monoclonal antibodies. Drug Discov Today Technol 21-22:75-83. https://doi.org/10.1016/j.ddtec.2016.09.004

122. Betts A, Keunecke A, van Steeg TJ, et al (2018) Linear pharmacokinetic parameters for monoclonal antibodies are similar within a species and across different pharmacological targets: A comparison between human, cynomolgus monkey and hFcRn Tg32 transgenic mouse using a population-modeling approach. mAbs 10:751-764. https://doi.org/10.1080/19420862.2018.1462429

123. Eigenmann MJ, Fronton L, Grimm HP, et al (2017) Quantification of IgG monoclonal antibody clearance in tissues. mAbs 9:1007-1015. https://doi.org/10.1080/19420862.2017.1337619

124. Butler A, Hoffman P, Smibert P, et al (2018) Integrating single-cell transcriptomic data across different conditions, technologies, and species. Nat Biotechnol 36:411420. https://doi.org/10.1038/nbt.4096 
125. Crinier A, Milpied P, Escalière B, et al (2018) High-Dimensional Single-Cell Analysis Identifies Organ-Specific Signatures and Conserved NK Cell Subsets in Humans and Mice. Immunity 49:971-986.e5. https://doi.org/10.1016/j.immuni.2018.09.009

126. Brunner KT, Mauel J, Cerottini JC, Chapuis B (1968) Quantitative assay of the lytic action of immune lymphoid cells on 51-Cr-labelled allogeneic target cells in vitro; inhibition by isoantibody and by drugs. Immunology 14:181-196

127. Cederbrant K (2005) Natural Killer Cell Assay. In: Vohr H-W (ed) Encyclopedic Reference of Immunotoxicology. Springer-Verlag, Berlin/Heidelberg, pp 469-472

128. Sorkin LS, Otto M, Baldwin WM, et al (2010) Anti-GD2 with an FC point mutation reduces complement fixation and decreases antibody-induced allodynia: Pain 149:135-142. https://doi.org/10.1016/j.pain.2010.01.024

129. Alderson KL, Luangrath M, Elsenheimer MM, et al (2013) Enhancement of the anti-melanoma response of Hu14.18K322A by $\alpha \mathrm{CD} 40+\mathrm{CpG}$. Cancer Immunol Immunother 62:665-675. https://doi.org/10.1007/s00262-012-1372-8

130. Hank JA, Surfus J, Gan J, et al (1994) Treatment of neuroblastoma patients with antiganglioside GD2 antibody plus interleukin-2 induces antibody-dependent cellular cytotoxicity against neuroblastoma detected in vitro. J Immunother Emphas Tumor Immunol Off J Soc Biol Ther 15:29-37

131. Henney CS, Kuribayashi K, Kern DE, Gillis S (1981) Interleukin-2 augments natural killer cell activity. Nature 291:335-338. https://doi.org/10.1038/291335a0

132. Rossi AR, Pericle F, Rashleigh S, et al (1994) Lysis of neuroblastoma cell lines by human natural killer cells activated by interleukin-2 and interleukin-12. Blood $83: 1323-1328$

133. Schulz G, Cheresh DA, Varki NM, et al (1984) Detection of ganglioside GD2 in tumor tissues and sera of neuroblastoma patients. Cancer Res 44:5914-5920

134. Nguyen R, Houston J, Chan WK, et al (2018) The role of interleukin-2, all-trans retinoic acid, and natural killer cells: surveillance mechanisms in anti-GD2 antibody therapy in neuroblastoma. Cancer Immunol Immunother CII 67:615-626. https://doi.org/10.1007/s00262-017-2108-6

135. Ladenstein R, Pötschger U, Valteau-Couanet D, et al (2018) Interleukin 2 with antiGD2 antibody ch14.18/CHO (dinutuximab beta) in patients with high-risk neuroblastoma (HR-NBL1/SIOPEN): a multicentre, randomised, phase 3 trial. Lancet Oncol 19:1617-1629. https://doi.org/10.1016/S1470-2045(18)30578-3

136. Moga E, Alvarez E, Cantó E, et al (2008) NK cells stimulated with IL-15 or CpG ODN enhance rituximab-dependent cellular cytotoxicity against B-cell lymphoma. Exp Hematol 36:69-77. https://doi.org/10.1016/j.exphem.2007.08.012 
137. Vincent M, Bessard A, Cochonneau D, et al (2013) Tumor targeting of the IL-15 superagonist RLI by an anti-GD2 antibody strongly enhances its antitumor potency. Int J Cancer 133:757-765. https://doi.org/10.1002/ijc.28059

138. Rosario M, Liu B, Kong L, et al (2016) The IL-15-Based ALT-803 Complex Enhances Fc $\gamma$ RIIIa-Triggered NK Cell Responses and In Vivo Clearance of B Cell Lymphomas. Clin Cancer Res Off J Am Assoc Cancer Res 22:596-608. https://doi.org/10.1158/1078-0432.CCR-15-1419

139. Neely GG, Robbins SM, Amankwah EK, et al (2001) Lipopolysaccharidestimulated or granulocyte-macrophage colony-stimulating factor-stimulated monocytes rapidly express biologically active IL-15 on their cell surface independent of new protein synthesis. J Immunol Baltim Md 1950 167:5011-5017

140. Ribeiro RC, Rill D, Roberson PK, et al (1993) Continuous infusion of interleukin-2 in children with refractory malignancies. Cancer 72:623-628

141. Conlon KC, Lugli E, Welles HC, et al (2015) Redistribution, hyperproliferation, activation of natural killer cells and CD8 T cells, and cytokine production during first-in-human clinical trial of recombinant human interleukin-15 in patients with cancer. J Clin Oncol Off J Am Soc Clin Oncol 33:74-82.

https://doi.org/10.1200/JCO.2014.57.3329

142. Allavena P, Giardina G, Bianchi G, Mantovani A (1997) IL-15 is chemotactic for natural killer cells and stimulates their adhesion to vascular endothelium. J Leukoc Biol 61:729-735

143. Hwang WL, Wolfson RL, Niemierko A, et al (2018) Clinical Impact of Tumor Mutational Burden in Neuroblastoma. JNCI J Natl Cancer Inst. https://doi.org/10.1093/jnci/djy157

144. Pugh TJ, Morozova O, Attiyeh EF, et al (2013) The genetic landscape of high-risk neuroblastoma. Nat Genet 45:279-284. https://doi.org/10.1038/ng.2529

145. Zhang P, Wu X, Basu M, et al (2017) MYCN Amplification Is Associated with Repressed Cellular Immunity in Neuroblastoma: An In Silico Immunological Analysis of TARGET Database. Front Immunol 8:1473. https://doi.org/10.3389/fimmu.2017.01473

146. Giri JG, Ahdieh M, Eisenman J, et al (1994) Utilization of the beta and gamma chains of the IL-2 receptor by the novel cytokine IL-15. EMBO J 13:2822-2830

147. Dubois S, Mariner J, Waldmann TA, Tagaya Y (2002) IL-15Ralpha recycles and presents IL-15 In trans to neighboring cells. Immunity 17:537-547

148. Pillet A-H, Thèze J, Rose T (2011) Interleukin (IL)-2 and IL-15 have different effects on human natural killer lymphocytes. Hum Immunol 72:1013-1017. https://doi.org/10.1016/j.humimm.2011.07.311 
149. Tamzalit F, Barbieux I, Plet A, et al (2014) IL-15.IL-15R complex shedding following trans-presentation is essential for the survival of IL-15 responding NK and T cells. Proc Natl Acad Sci 111:8565-8570. https://doi.org/10.1073/pnas.1405514111

150. Neely GG, Robbins SM, Amankwah EK, et al (2001) LipopolysaccharideStimulated or Granulocyte-Macrophage Colony-Stimulating Factor-Stimulated Monocytes Rapidly Express Biologically Active IL-15 on Their Cell Surface Independent of New Protein Synthesis. J Immunol 167:5011-5017. https://doi.org/10.4049/jimmunol.167.9.5011

151. Yu AL, Gilman AL, Ozkaynak MF, et al (2010) Anti-GD2 antibody with GM-CSF, interleukin-2, and isotretinoin for neuroblastoma. N Engl J Med 363:1324-1334. https://doi.org/10.1056/NEJMoa0911123

152. Nguyen QH, Roberts RL, Ank BJ, et al (1998) Interleukin (IL)-15 enhances antibody-dependent cellular cytotoxicity and natural killer activity in neonatal cells. Cell Immunol 185:83-92. https://doi.org/10.1006/cimm.1998.1286

153. Moga E, Alvarez E, Cantó E, et al (2008) NK cells stimulated with IL-15 or CpG ODN enhance rituximab-dependent cellular cytotoxicity against B-cell lymphoma. Exp Hematol 36:69-77. https://doi.org/10.1016/j.exphem.2007.08.012

154. Vincent M, Bessard A, Cochonneau D, et al (2013) Tumor targeting of the IL-15 superagonist RLI by an anti-GD2 antibody strongly enhances its antitumor potency. Int J Cancer 133:757-765. https://doi.org/10.1002/ijc.28059

155. Sobolev O, Stern P, Lacy-Hulbert A, Hynes RO (2009) Natural Killer Cells Require Selectins for Suppression of Subcutaneous Tumors. Cancer Res 69:2531-2539. https://doi.org/10.1158/0008-5472.CAN-08-3126

156. Son BK, Roberts RL, Ank BJ, Stiehm ER (1996) Effects of anticoagulant, serum, and temperature on the natural killer activity of human peripheral blood mononuclear cells stored overnight. Clin Diagn Lab Immunol 3:260-264

157. Cheung N-KV, Sowers R, Vickers AJ, et al (2006) FCGR2A polymorphism is correlated with clinical outcome after immunotherapy of neuroblastoma with antiGD2 antibody and granulocyte macrophage colony-stimulating factor. J Clin Oncol Off J Am Soc Clin Oncol 24:2885-2890. https://doi.org/10.1200/JCO.2005.04.6011

158. Delgado DC, Hank JA, Kolesar J, et al (2010) Genotypes of NK Cell KIR Receptors, Their Ligands, and Fc Receptors in the Response of Neuroblastoma Patients to Hu14.18-IL2 Immunotherapy. Cancer Res 70:9554-9561. https://doi.org/10.1158/0008-5472.CAN-10-2211

159. Forlenza CJ, Boudreau JE, Zheng J, et al (2016) KIR3DL1 Allelic Polymorphism and HLA-B Epitopes Modulate Response to Anti-GD2 Monoclonal Antibody in 
Patients With Neuroblastoma. J Clin Oncol Off J Am Soc Clin Oncol 34:24432451. https://doi.org/10.1200/JCO.2015.64.9558

160. Erbe AK, Wang W, Carmichael L, et al (2018) Neuroblastoma Patients' KIR and KIR-Ligand Genotypes Influence Clinical Outcome for Dinutuximab-based Immunotherapy: A Report from the Children's Oncology Group. Clin Cancer Res 24:189-196. https://doi.org/10.1158/1078-0432.CCR-17-1767

161. Grimm EA, Mazumder A, Zhang HZ, Rosenberg SA (1982) Lymphokine-activated killer cell phenomenon. Lysis of natural killer-resistant fresh solid tumor cells by interleukin 2-activated autologous human peripheral blood lymphocytes. J Exp Med 155:1823-1841

162. Hank JA, Robinson RR, Surfus J, et al (1990) Augmentation of antibody dependent cell mediated cytotoxicity following in vivo therapy with recombinant interleukin 2. Cancer Res 50:5234-5239

163. Atkins MB, Gould JA, Allegretta M, et al (1986) Phase I evaluation of recombinant interleukin-2 in patients with advanced malignant disease. J Clin Oncol 4:13801391. https://doi.org/10.1200/JCO.1986.4.9.1380

164. Lode H, Siebert N, Kietz S, et al (2013) Long-term continuous infusion of anti-GD2 antibody $\mathrm{CH} 14.18 / \mathrm{CHO}$ in relapsed/refractory neuroblastoma patients. J Immunother Cancer 1:P244. https://doi.org/10.1186/2051-1426-1-S1-P244

165. Delgado DC, Hank JA, Kolesar J, et al (2010) Genotypes of NK Cell KIR Receptors, Their Ligands, and Fc Receptors in the Response of Neuroblastoma Patients to Hu14.18-IL2 Immunotherapy. Cancer Res 70:9554-9561. https://doi.org/10.1158/0008-5472.CAN-10-2211

166. Venstrom JM, Zheng J, Noor N, et al (2009) KIR and HLA genotypes are associated with disease progression and survival following autologous hematopoietic stem cell transplantation for high-risk neuroblastoma. Clin Cancer Res Off J Am Assoc Cancer Res 15:7330-7334. https://doi.org/10.1158/10780432.CCR-09-1720

167. Marsh SGE, Parham P, Barber LD (2000) The HLA factsbook. Academic Press, San Diego

168. Jin HJ, Nam HY, Bae YK, et al (2010) GD2 expression is closely associated with neuronal differentiation of human umbilical cord blood-derived mesenchymal stem cells. Cell Mol Life Sci 67:1845-1858. https://doi.org/10.1007/s00018-010-0292-z

169. Yu AL, Gilman AL, Ozkaynak MF, et al (2010) Anti-GD2 Antibody with GMCSF, Interleukin-2, and Isotretinoin for Neuroblastoma. N Engl J Med 363:13241334. https://doi.org/10.1056/NEJMoa0911123 
170. Zou Z, Nomura M, Takihara $Y$, et al (1996) Isolation and characterization of retinoic acid-inducible cDNA clones in F9 cells: a novel cDNA family encodes cell surface proteins sharing partial homology with MHC class I molecules. J Biochem (Tokyo) 119:319-328

171. Ho EL, Carayannopoulos LN, Poursine-Laurent J, et al (2002) Costimulation of Multiple NK Cell Activation Receptors by NKG2D. J Immunol 169:3667-3675. https://doi.org/10.4049/jimmunol.169.7.3667

172. Reynolds CP, Kane DJ, Einhorn PA, et al (1991) Response of neuroblastoma to retinoic acid in vitro and in vivo. Prog Clin Biol Res 366:203-211

173. Schug TT, Berry DC, Shaw NS, et al (2007) Opposing effects of retinoic acid on cell growth result from alternate activation of two different nuclear receptors. Cell 129:723-733. https://doi.org/10.1016/j.cell.2007.02.050

174. Lin J-X, Du N, Li P, et al (2017) Critical functions for STAT5 tetramers in the maturation and survival of natural killer cells. Nat Commun 8:. https://doi.org/10.1038/s41467-017-01477-5

175. Stoklasek TA, Schluns KS, Lefrançois L (2006) Combined IL-15/IL-15Ralpha immunotherapy maximizes IL-15 activity in vivo. J Immunol Baltim Md 1950 177:6072-6080

176. Rubinstein MP, Kovar M, Purton JF, et al (2006) Converting IL-15 to a superagonist by binding to soluble IL-15R \{alpha\}. Proc Natl Acad Sci U S A 103:9166-9171. https://doi.org/10.1073/pnas.0600240103

177. Rhode PR, Egan JO, Xu W, et al (2016) Comparison of the Superagonist Complex, ALT-803, to IL15 as Cancer Immunotherapeutics in Animal Models. Cancer Immunol Res 4:49-60. https://doi.org/10.1158/2326-6066.CIR-15-0093-T

178. Romee R, Cooley S, Berrien-Elliott MM, et al (2018) First-in-human phase 1 clinical study of the IL-15 superagonist complex ALT-803 to treat relapse after transplantation. Blood 131:2515-2527. https://doi.org/10.1182/blood-2017-12823757

179. Mody R, Naranjo A, Van Ryn C, et al (2017) Irinotecan-temozolomide with temsirolimus or dinutuximab in children with refractory or relapsed neuroblastoma (COG ANBL1221): an open-label, randomised, phase 2 trial. Lancet Oncol 18:946957. https://doi.org/10.1016/S1470-2045(17)30355-8

180. Liu Y, Wu H-W, Sheard MA, et al (2013) Growth and activation of natural killer cells ex vivo from children with neuroblastoma for adoptive cell therapy. Clin Cancer Res Off J Am Assoc Cancer Res 19:2132-2143. https://doi.org/10.1158/1078-0432.CCR-12-1243 
181. Barry WE, Jackson JR, Asuelime GE, et al (2018) Activated Natural Killer Cells in Combination with Anti-GD2 Antibody Dinutuximab Improve Survival of Mice after Surgical Resection of Primary Neuroblastoma. Clin Cancer Res Off J Am Assoc Cancer Res. https://doi.org/10.1158/1078-0432.CCR-18-1317

182. Stewart E, Shelat A, Bradley C, et al (2015) Development and characterization of a human orthotopic neuroblastoma xenograft. Dev Biol 407:344-355. https://doi.org/10.1016/j.ydbio.2015.02.002

183. Almeida-Oliveira A, Smith-Carvalho M, Porto LC, et al (2011) Age-related changes in natural killer cell receptors from childhood through old age. Hum Immunol 72:319-329. https://doi.org/10.1016/j.humimm.2011.01.009

184. Le Garff-Tavernier M, Béziat V, Decocq J, et al (2010) Human NK cells display major phenotypic and functional changes over the life span: Human NK cell features over the life span. Aging Cell 9:527-535. https://doi.org/10.1111/j.14749726.2010.00584.x

185. Lutz CT, Moore MB, Bradley S, et al (2005) Reciprocal age related change in natural killer cell receptors for MHC class I. Mech Ageing Dev 126:722-731. https://doi.org/10.1016/j.mad.2005.01.004

186. Markasz L, Stuber G, Vanherberghen B, et al (2007) Effect of frequently used chemotherapeutic drugs on the cytotoxic activity of human natural killer cells. Mol Cancer Ther 6:644-654. https://doi.org/10.1158/1535-7163.MCT-06-0358

187. Rujkijyanont $\mathrm{P}$, Chan WK, Eldridge PW, et al (2013) Ex vivo activation of CD56(+) immune cells that eradicate neuroblastoma. Cancer Res 73:2608-2618. https://doi.org/10.1158/0008-5472.CAN-12-3322

188. Bach C, Steffen M, Roesler W, et al (2017) Systematic comparison of donor chimerism in peripheral blood and bone marrow after hematopoietic stem cell transplantation. Blood Cancer J 7:e566. https://doi.org/10.1038/bcj.2017.42

189. Kenderian SS, Ruella M, Shestova O, et al (2015) CD33-specific chimeric antigen receptor $\mathrm{T}$ cells exhibit potent preclinical activity against human acute myeloid leukemia. Leukemia 29:1637-1647. https://doi.org/10.1038/leu.2015.52 


\section{VITA}

Rosa Nguyen was born in Hamburg, Germany in 1985. She earned her Doctor of Medicine degree from the University of Hamburg in 2010. She completed her pediatric residency at the University of Maryland in 2014 and her pediatric sub-specialty training at St. Jude Children's Research Hospital in 2017. She is a board-certified pediatrician and board-eligible in pediatric hematology-oncology. She was matriculated in the Ph.D. program in Biomedical Sciences (IBSP) at the University of Tennessee Health Science Center in Memphis in August 2015. She received a research grant by the German Cancer Foundation in 2011 and was awarded the Conquer Cancer ASCO Young Investigator Award in 2018. 